\title{
Effiziente Anheizung von Gebäuden in massiver Bauweise bei intermittierender Beheizung
}

\author{
zur Erlangung des akademischen Grades Doktor-Ingenieur \\ an der Fakultät für Architektur und Bauingenieurwesen \\ der technischen Universität Dortmund \\ genehmigte Dissertation \\ von \\ Alexander Siebel \\ Aachen
}

1. Gutachter:

Univ.-Prof. Dr.-Ing. habil. Wolfgang M. Willems

2. Gutachter: Univ.-Prof. Dr.-Ing. Peter Schmidt, Institut für konstruktiven Ingenieurbau der Universität Siegen

Tag der Einreichung:

27. September 2012

Tag der mündlichen Prüfung:

5. März 2013

Aachen

8. März 2013 



\section{Vorwort}

Die vorliegende Arbeit entstand am Lehrstuhl Bauphysik und Technische Gebäudeausrüstung der Technischen Universität Dortmund. Mein Dank gilt insbesondere Herrn Univ.-Prof. Dr.-Ing. habil. Wolfgang M. Willems und Herrn Univ.-Prof. Dr.-Ing. Peter Schmidt für die Betreuung, Unterstützung und Geduld während der Erstellung dieser Arbeit. Sie waren bei der Erstellung der vorliegenden Arbeit konstruktive Diskussionspartner und es fand ein praxisnaher Gedankenaustausch statt. Für die Unterstützung bei den technischen Darstellungen danke ich Frau Kerstin Hübner vom Lehrstuhl Bauphysik und Technische Gebäudeausrüstung an der Technischen Universität Dortmund.

Die Erstellung der Arbeit erfolgte begleitend zu meiner Berufstätigkeit als Ingenieur bei der SWA GmbH in Aachen. Die SWA GmbH ist unter anderem im Bereich der Baustoff- und Bauproduktprüfung tätig. Hierdurch konnten viel Praxiswissen und messtechnische Erfahrung in die Arbeit einfließen. In diesem Zusammenhang bedanke ich mich bei meinem Vater, der mir stets ein konstruktiver und kritischer Diskussionspartner war.

Besondere Hilfe wurde mir zudem durch Herrn Dipl.-Ing. Stefan Braun von der Firma SmartCAE aus München zuteil, welcher mich bei der Simulationsberechnung dünner Schichten unterstützte. Mit der Software „HeatTransmission“ konnten auch Temperaturfelder zu den durchgeführten Labor- und Objektmessungen simuliert werden. Dank der sehr guten Unterstützung durch Herrn Dipl.-Ing. Stefan Braun wurde das Programm auf die in der vorliegenden Arbeit benötigten Bedingungen angepasst.

Letztlich möchte ich noch allen danken, welche mir Räume für messtechnische Untersuchungen zur Verfügung gestellt haben und Unannehmlichkeiten während der Messungen in ihren normal benutzen Räumen in Kauf genommen haben. 


\section{Inhaltsverzeichnis}

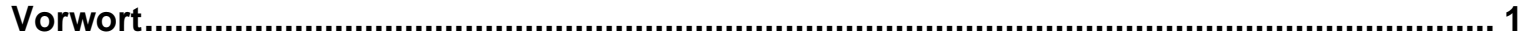

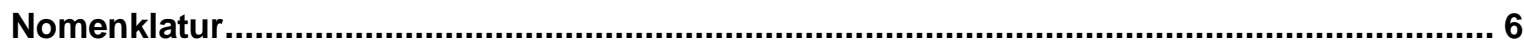

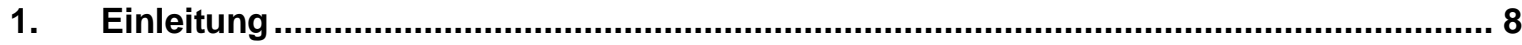

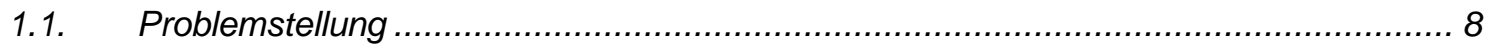

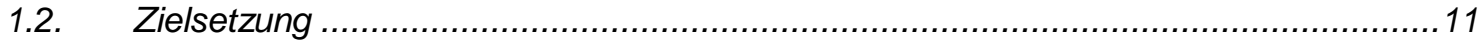

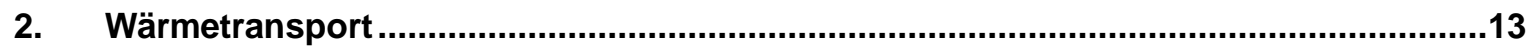

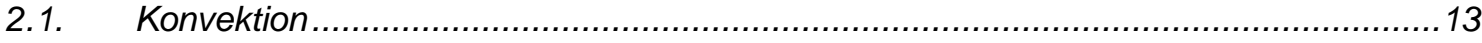

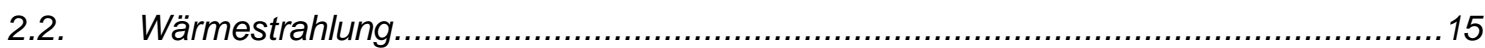

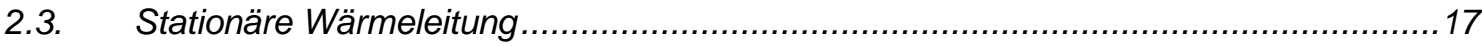

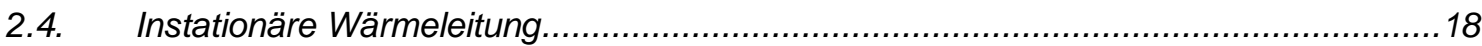

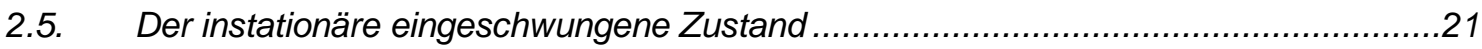

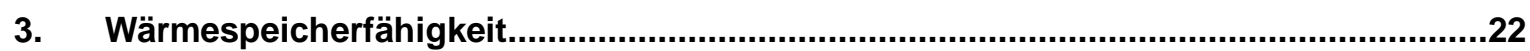

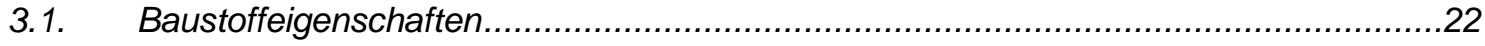

3.2. Bauteileigenschaften unter instationären Randbedingungen ...................................26

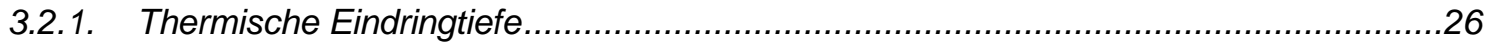

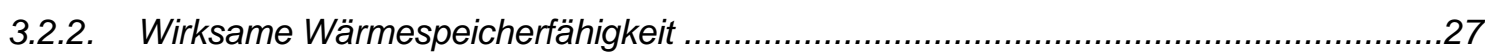

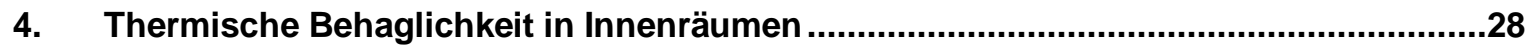

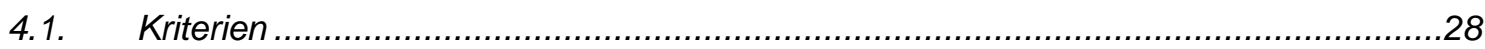

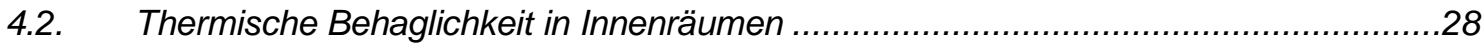

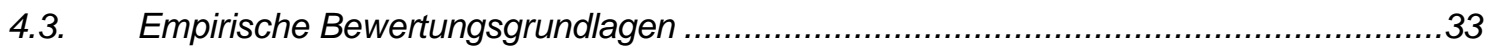

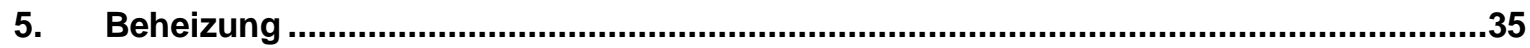

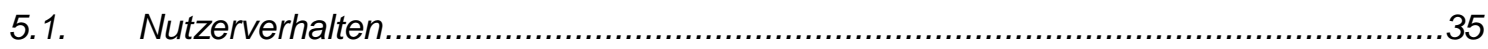

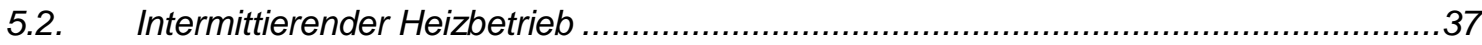

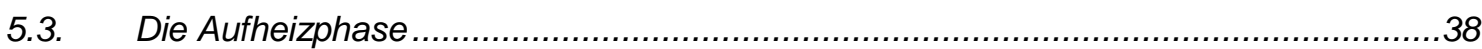

6. Messtechnische Untersuchung der thermischen Entwicklung in Räumen während

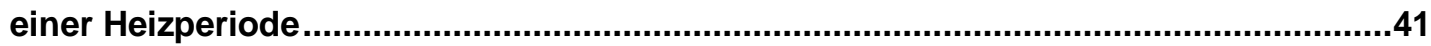

6.1. Vorgehensweise und Randbedingungen ......................................................... 


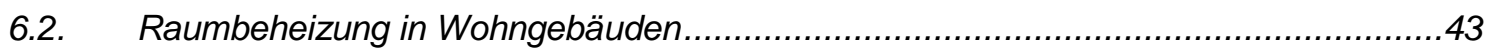

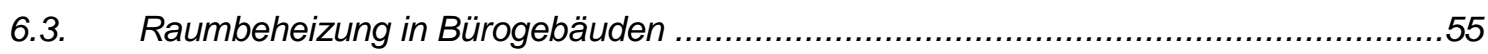

6.4. Zusammenfassung der vorgefundenen Verhältnisse .............................................58

7. Messtechnische Untersuchung dünner Dämmschichten an massiven

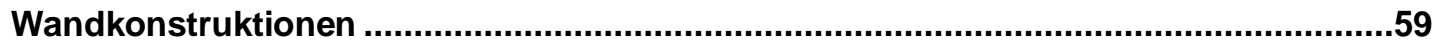

7.1. Anheizphase einer massiven Wandkonstruktion unter Laborbedingungen ..................59

7.1.1. Anheizphase im Labor-Prüfstand für eine einmalige Anheizung ..............................63

7.1.2. Anheizphase im Labor-Prüfstand bei einer intermittierenden Beheizung ...................64

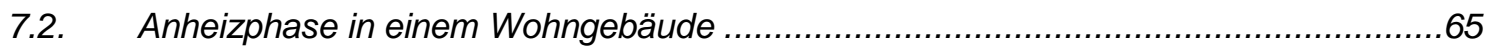

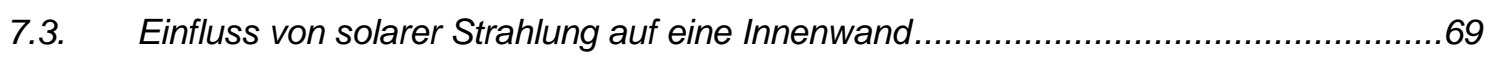

7.4. Lüftungseinfluss auf die operative Temperatur ................................................... 72

7.5. Energieeinsparung bei einem Wohngebäude ................................................... 74

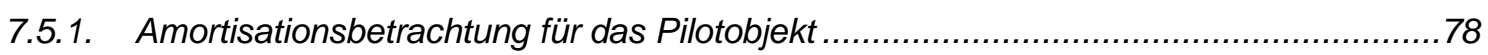

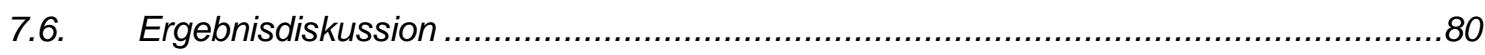

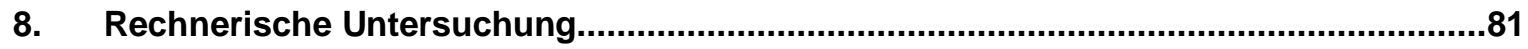

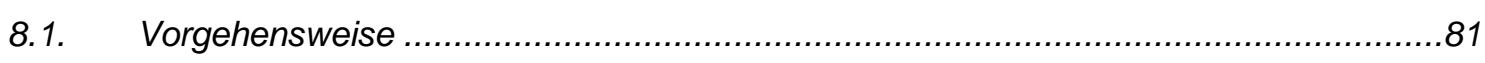

8.2. Validierung der Berechnungen mit der Messergebnissen ......................................81

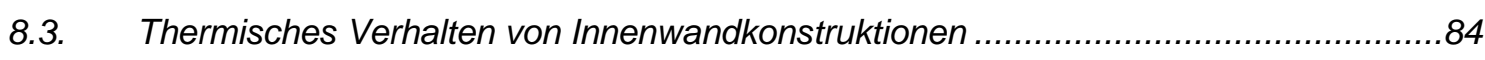

8.3.1. Innenwandkonstruktionen bei instationärem Heizbetrieb..........................................85

8.4. Thermisches Verhalten von Außenwandkonstruktionen .........................................96

8.5. Berechnung der gespeicherten Energie bei intermittierendem Heizbetrieb................102

8.5.1. Gespeicherte Energiemenge bei intermittierendem Heizbetrieb .............................107

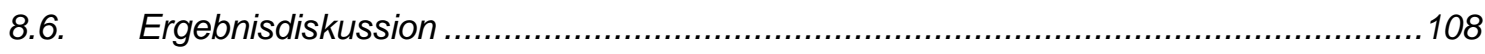

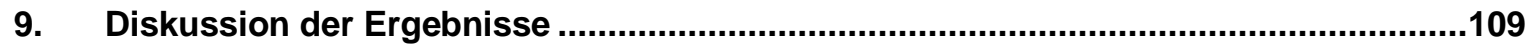

9.1. Temperierung von realen Wohn- und Büroräumen .............................................109

9.2. Messtechnisch und rechnerisch untersuchter Einfluss dünner Dämmschichten an

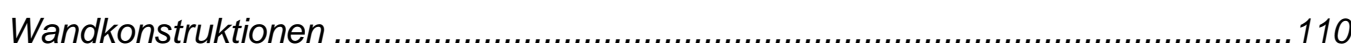

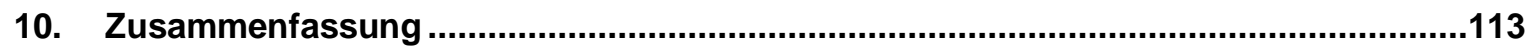

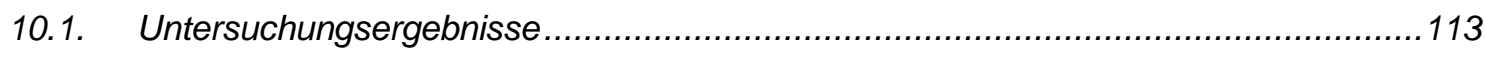

10.2. Vorteile für das Raumklima bei entsprechenden Maßnahmen.................................113 
10.3. Möglichkeiten der Energieeinsparung ..........................................................113

10.4. Empfehlung zur Anwendung in der Praxis ........................................................114

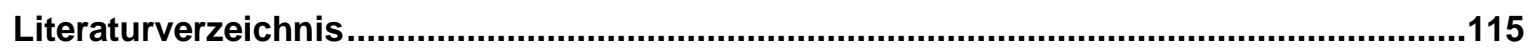

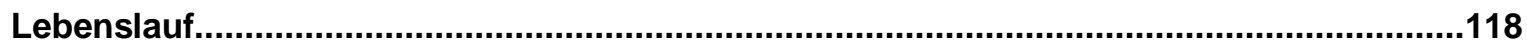




\section{Nomenklatur}

\section{Verwendete Formelzeichen}

A [m²] Fläche, Querschnittsfläche

a $\quad\left[\frac{m^{2}}{s}\right] \quad$ Temperaturleitkoeffizient

$A_{i} \quad\left[m^{2}\right] \quad$ Teilfläche

$A_{W} \quad\left[m^{2}\right] \quad$ Oberfläche der wärmespeichernden Bauteile

b $\quad\left[\frac{W}{m^{2} \cdot s^{0,5}}\right] \quad$ Wärmeeindringkoeffizient

C $\quad\left[\frac{\mathrm{J}}{\mathrm{kg} \cdot \mathrm{m}^{3}}\right] \quad$ spezifische Wärmekapazität

clo $[-] \quad$ thermischer Widerstand von Personen-Bekleidungen

d $\quad[\mathrm{cm}, \mathrm{m}] \quad$ Schichtdicke

$\Delta \mathrm{T} \quad[\mathrm{K}] \quad$ Temperaturdifferenz

$\varepsilon \quad[-] \quad$ thermischer Emissionsgrad

$\mathrm{f}_{\mathrm{RH}} \quad[-] \quad$ Korrekturfaktor abhängig von einer gewünschten Aufheizzeit

hc [W] konvektiver Wärmeübergangskoeffizient

$\lambda \quad\left[\frac{W}{m \cdot k}\right] \quad$ Wärmeleitfähigkeit

m $\quad[\mathrm{kg}] \quad$ Masse

met [-] Wärmeproduktion einer Person in Abhängigkeit von ihrer Tätigkeit

PPD [\%] Predicted Percentage of Dissatisfied

$\phi_{\mathrm{RH}, \mathrm{i}} \quad[\mathrm{W}] \quad$ zusätzlich benötigte Aufheizleistung

$\phi_{T, \mathrm{i}} \quad[\mathrm{W}] \quad$ stationärer Transmissionswärmeverlust

$\phi_{\mathrm{v}, \mathrm{i}} \quad[\mathrm{W}] \quad$ Lüftungswärmeverlust

[6] 

Q $\quad[\mathrm{J}, \mathrm{kWh}] \quad$ Wärmemenge
$\mathrm{Q}_{\mathrm{F}} \quad[\mathrm{kWh}] \quad$ Wärmeverluste durch Fenster und andere kaum wärmespeichernde Bauteile
$\mathrm{Q}_{W} \quad[\mathrm{kWh}] \quad$ Wärmebedarf von stark wärmespeichernden Bauteilen
$Q_{L} \quad[k W h] \quad$ Lüftungswärmeverlust in der Anheizphase
$\mathrm{q}_{\mathrm{c}} \quad\left[\frac{\mathrm{W}}{\mathrm{m}^{2}}\right] \quad$ konvektiv bedingte Wärmestromdichte
$\rho \quad\left[\frac{\mathrm{kg}}{\mathrm{m}^{3}}\right] \quad$ Baustoffdichte
$\mathrm{R} \quad\left[\frac{K^{\prime} \cdot m^{2}}{W}\right] \quad$ Wärmedurchlasswiderstandskoeffizient
S $\quad\left[\frac{W}{m^{2} \cdot K}\right] \quad$ Wärmespeicherwert
t $\quad[s, h, \min ] \quad$ Zeit
$\mathrm{T} \quad\left[{ }^{\circ} \mathrm{C}\right] \quad$ absolute Temperatur
$\begin{array}{lll}\theta & {\left[{ }^{\circ} \mathrm{C}\right]} & \text { Temperatur }\end{array}$
$\theta_{\mathrm{a}} \quad\left[{ }^{\circ} \mathrm{C}\right] \quad$ Raumluftemperatur
$\theta_{\circ} \quad\left[{ }^{\circ} \mathrm{C}\right] \quad$ operative Temperatur
$\begin{array}{lll}\theta_{\mathrm{r}} & {\left[{ }^{\circ} \mathrm{C}\right] \quad \text { mittlere Oberflächentemperatur }}\end{array}$
$\theta_{\mathrm{si}} \quad\left[{ }^{\circ} \mathrm{C}\right] \quad$ Oberflächentemperatur
$\mathrm{x} \quad[\mathrm{m}] \quad$ Entfernung, Ort

\section{Mathematische Symbole}

$\delta \quad$ Operator für partielles Differential

$\Delta \quad$ Differenzenoperator

$\Sigma$ Summe 


\section{Einleitung}

\subsection{Problemstellung}

In der Heizperiode ist zur Deckung des Wärmebedarfs in Wohn- und Arbeitsräumen eine Beheizung notwendig, um behagliche Raumtemperaturen herzustellen.

Der Wärmebedarf eines Raumes beziehungsweise eines Gebäudes hängt dabei vereinfacht betrachtet von den internen und externen Energieeinträgen, dem effektiv wirksamen Wärmedämmniveau der Gebäudehülle, der Belüftung der Räume sowie von der Effizienz des Heizungssystems einschließlich seiner Regelung ab. Das Raumklima wird grundsätzlich vom Außenklima sowie der Beheizung, welche durch den Nutzer geregelt wird, bestimmt. In der Praxis liegen keine stationären Randbedingungen vor, weil das Außenklima ständig wechselnde Lufttemperaturen, Sonneneinstrahlungsintensitäten, Luftbewegungen und Luftfeuchtigkeiten aufweist und zeitweilige Raumlufttemperaturabsenkungen normal sind. Aufgrund der instationären Klimabedingungen des Außen- und Raumklimas ist neben dem Wärmedurchgang auch die Wärmespeicherfähigkeit der Bauteile von Bedeutung, vergleiche [1] [2] [3] und [4]. Die wirksame Wärmespeicherfähigkeit kennzeichnet die Wärmemengen, die ein Stoff, abhängig von seiner Temperaturniveaudifferenz, aufnimmt.

Ein Gebäude setzt sich aus unterschiedlichen Bauteilen zusammen. Diese wiederum bestehen aus Bauelementen und -schichten, welche das Raumklima durch ihre Wärmeleitfähigkeit und -speicherfähigkeit beeinflussen. Bei den Wand- und Deckenkonstruktionen unterscheidet man grob zwischen einer massiven oder leichten Bauweise. Unter einer massiven Bauweise versteht man ein vorwiegend aus gemauerten oder betonierten Konstruktionen bestehendes Bauwerk. Bei einer leichten Bauweise bestehen die Wände und/oder Decken aus schlankeren Tragkonstruktionen (zum Beispiel aus einem Ständerwerk) und aus dünnen Verkleidungen (zum Beispiel aus Gipskartonplatten). Oftmals sind in einem Bauwerk beide Konstruktionsvarianten vorhanden. In Mitteleuropa werden die normalen Vollgeschosse vorwiegend massiv und die Bauteile im Steildachbereich zum größten Teil in Leichtbauweise errichtet.

Im Massivbau wird das „Wechselklima“ über Stunden und Tage ausgeglichen. Keller [5] beschreibt die Temperaturentwicklung im Bauteil als „thermische Geschichte“.

Die unterschiedliche Wärmespeicherfähigkeit einer massiven und leichten Baukonstruktion wird deutlich, wenn man eine Heizungsunterbrechung beispielsweise während der Nachtzeit oder am Wochenende betrachtet. Eine Leichtbaukonstruktion kühlt schneller aus als eine massive Baukonstruktion. Sie wärmt sich bei einer Wiederanheizung jedoch auch schneller wieder auf. Aus diesem Grund ist der Energieverbrauch infolge einer intermittierenden 
Beheizung bei einer leichten und einer massiven Baukonstruktion abhängig von der Dauer der Temperaturabsenkung. Untersuchungen von Hauser [6] haben gezeigt, wie sich Gebäude von massiver und leichter Bauweise bei einem intermittierenden Heizbetrieb energetisch unterscheiden. Hier ergibt sich beim Leichtbau - gerade bei längeren Heizungsunterbrechungen - ein größeres Energieeinsparpotenzial als beim Massivbau.

In der Vergangenheit war eine massive Bauweise wegen der Beheizung mit Einzelöfen erwünscht. Die Wärmespeicherfähigkeit der Bauteile diente dabei zum Ausgleich der Temperaturschwankungen, welche durch den nicht kontinuierlichen Wärmeeintrag infolge von handbetriebenen Einzelöfen vorlagen [7].

In der Zeit zwischen 1952 - 1981 wurden durch die DIN 4108, auch wegen der nicht kontinuierlichen Beheizung, höhere Anforderungen an die Wärmedämmung von leichten Außenbauteilen gestellt. Bei Außenbauteilen mit Massen unter $200 \mathrm{~kg} / \mathrm{m}^{2}$ forderte man eine Beheizung mittels wärmespeichernden Öfen (Kachelöfen) oder eine sehr kontinuierliche Beheizung mittels einer Zentralheizung [7].

Durch den Komfort einer Zentralheizung verloren die Einzelöfen und damit die starken Anheiz- und Auskühlphasen (bei kontinuierlicher Nutzung) an Bedeutung. Beim Betrieb der Zentralheizung erfolgt im Gegensatz zur früheren Einzelofenbeheizung eine vergleichsweise stationäre Beheizung der Gebäude [7].

\begin{abstract}
„Bei der heutigen stationären Beheizung hat die Wärmespeicherfähigkeit von Gebäuden im Hinblick auf winterliche Verhältnisse keine nennenswerte Bedeutung mehr, eher hinsichtlich der Verhältnisse im Sommer, wobei aber dem Einfluss der Sonneneinstrahlung durch Fenster und einem wirksamen Sonnenschutz der Haupteinfluss zukommt. Trotzdem wird eine große Wärmespeicherfähigkeit auch heute noch sehr hoch bewertet. Das geht so weit, dass in der Vergangenheit manchmal behauptet worden ist, die Wärmespeicherfähigkeit sei wichtiger als die Wärmedämmung. " [7]
\end{abstract}

Vor dem Hintergrund permanent steigender Energiekosten und dem Umdenken in der Gesellschaft von einen hemmungslosen zu einem behutsameren Umgang mit fossilen Brennstoffen fordert die Energieeinsparverordnung seit jüngerer Zeit ein sehr hohes Dämmniveau beim Neubau, bei Nutzungsänderungen, Gebäudeerweiterungen sowie eine gewisse Nachrüstung im Bestand [8] [9] [10] [11] [12] [13] [14].

Bei üblichen Wärmedämmmaßnahmen - im Sinne der Energieeinsparverordnung (EnEV) wird ein kontinuierlicher Heizbetrieb angenommen. Die Praxis sowie die Untersuchungsergebnisse zur Beheizung von Wohn- und Arbeitsräumen in Kapitel 6 zeigen, dass der Heizbetrieb oftmals unterbrochen beziehungsweise abgesenkt wird. 
Diese Heizungsunterbrechungen fordern eine erneute Wiederanheizung, um behagliche Raumluft- und Oberflächentemperaturen zu erzielen. Eine instationäre Betrachtung des Innenraumklimas hat bisher keinen Einzug in die Berechnungen der Energiebilanzen gefunden. Grund hierfür scheinen der Aufwand bei der Datenerfassung sowie die aufwendigere Berechnung zu sein.

Zudem müsste beim jeweiligen Objekt die Beheizungsart unter Berücksichtigung der Wärmeübergabesituation in den einzelnen Räumen genauer betrachtet werden.

In der Praxis wird die Raumlufttemperatur in Objekten bei einer unterschiedlichen Nutzung in den Tag- und Nachtzeiten während „Nichtnutzungszeiten“ abgesenkt, in der Erwartung damit Energie und Kosten einsparen zu können [15]. Der intermittierende Heizbetrieb verursacht allerdings infolge der „Wärmeträgheit“ folgende drei Phasen [16]:

- Raumanheizphase

- Raumnutzungsphase ggf. Behaglichkeitsphase

- Raumauskühlphase.

In den immer wieder auftretenden Raumanheizphasen müssen die Lufttemperatur und die Oberflächentemperaturen der raumumschließenden Bauteile in den Behaglichkeitsbereich gebracht werden [16]. Dieser Vorgang benötigt Energie und Zeit für die Wiederauffüllung der Wärmespeicher [17]. Zudem ist die Behaglichkeit in der Raumanheizphase aufgrund der kühlen raumumschließenden Bauteile sowie durch eine ggf. ungleichmäßige Temperaturverteilung nicht gegeben [16]. Daher erfolgt in der „Nichtnutzungszeit“ der Räume eine entsprechend lange Vorheizung (ohne Anwesenheit von Personen).

Ein für den „Anheizenergieaufwand“ vereinfachter Rechenansatz zur Dimensionierung von Heizkörpern wird in der DIN EN 12831 mit dem Wiederaufheizfaktor $f_{R H}$ beschrieben. In diesem Faktor wird die Bauweise pauschal als $\mathrm{I}=$ leicht oder $\mathrm{s}=$ mittelschwer/schwer eingestuft, um die benötigte Heizkörpernennleistung zu ermitteln. Diese Betrachtung stellt eine Dimensionierungsgrundlage für die Heizkörperauslegung unter Berücksichtigung der Wiederanheizung von Räumen dar. Eine Bewertung der Wärmespeicherung der raumumschlieBenden Bauteile in Hinblick auf einen energetisch optimierten Heizbetrieb und der daraus resultierenden Energieeinsparung oder -verschwendung wird dabei nicht vorgenommen. Es fehlt eine Methode zur Bewertung der Speichermassen hinsichtlich des Energieaufwandes zur Beheizung von Räumen in der Heizperiode, insbesondere dann, wenn thermisch flinkere, oberflächennahe Bauteilschichten oder auch Vorhänge oder Ähnliches verwendet werden. 


\subsection{Zielsetzung}

Im Vordergrund dieser Arbeit steht der Raumbeheizungs-Energieaufwand und die Bewertung der Anheizphase bis zur Erzielung der thermischen Behaglichkeit in Räumen. Ziel ist es, die Behaglichkeit im hier zu Lande üblichen Massivbau während der Heizperiode günstig zu gestalten und dabei Heizenergie einzusparen. Hierzu wird der Frage nachgegangen, inwieweit sich die Raumanheizphase durch sehr dünne Dämmschichten an den raumumschließenden Oberflächen der Bauteile verkürzen lässt. Der Einfluss einer Vliesschicht auf die thermische Entwicklung der Wandkonstruktion ist in der vorliegenden Arbeit messtechnisch und rechnerisch untersucht worden. Durch die Einbeziehung eines realen Objektes in die Versuche konnten die tatsächlichen Energieverbräuche bei der Verwendung einer entsprechenden Wandbeschichtung ermittelt werden. Die untersuchten Dämmschichten sind aufgrund ihrer geringen Schichtstärke nicht als Wärmedämmung zu betrachten und können keine Außen- oder Innendämmung ersetzen.

Untersucht wurden bevorzugt Dämmschichten aus Vliesmaterialien, welche sehr wasserdampfdurchlässig sind und damit einen günstigen Feuchtetransport ermöglichen, vergleiche Abbildung 1.1.
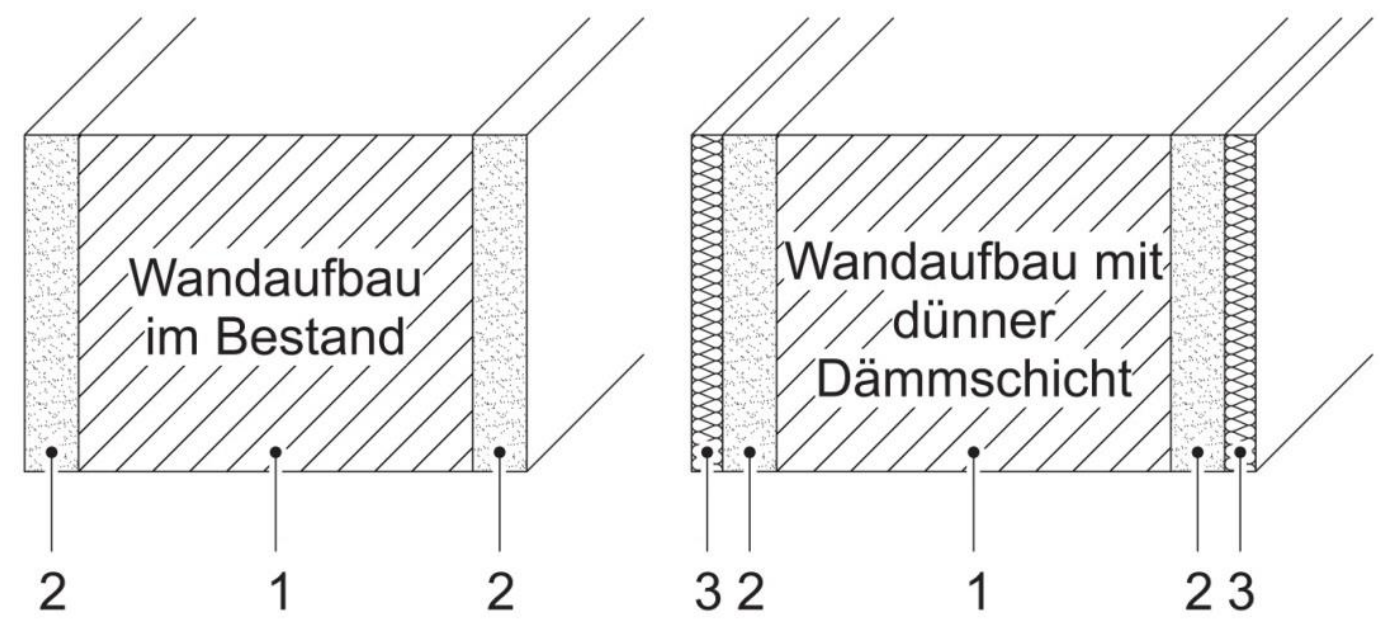

\section{Legende:}

1) Mauerwerk oder dergleichen

2) Putz

3) dünne Vliesschicht, bis zu ca. 4 mm Dicke

Abbildung 1.1: Schematischer Aufbau einer Innenwand mit und ohne dünne Dämmschichten

In der Abbildung 1.2 ist der Schichtaufbau auf einer Betonplatte mit einer Raufasertapete dargestellt. Die Vliesschicht wurde mit einem speziellen Dispersionskleber flächig auf der Betonplatte vergeklebt. Die Raufasertapete ist danach auf der Vliestapete mit einem han- 
delsüblichen Kleister aufgebracht worden. Bei einer späteren Renovierung im Objekt kann so die Vliesschicht auf der Wand erhalten bleiben. Die Decktapete - hier als Beispiel Raufaser - kann bei Bedarf erneuert oder wieder überstrichen werden. Durch die Vliesschicht werden auch kleinere Unebenheiten, wie zum Beispiel Löcher in der Wand, überdeckt, so dass die üblichen Vorarbeiten, zum Beispiel Spachtel- und Schleifarbeiten, verringert werden.

Die verwendete Vliesschicht unter der Tapete hat den Vorteil, dass ein Eindrücken der Schicht durch Punktlasten möglich ist, sich das Material wieder zurückstellt. Dieser Vorteil ist bei anderen Dämmstoffen, wie zum Beispiel Polystyrol, meistens nicht gegeben und es kommt zu bleibenden Eindrücken.

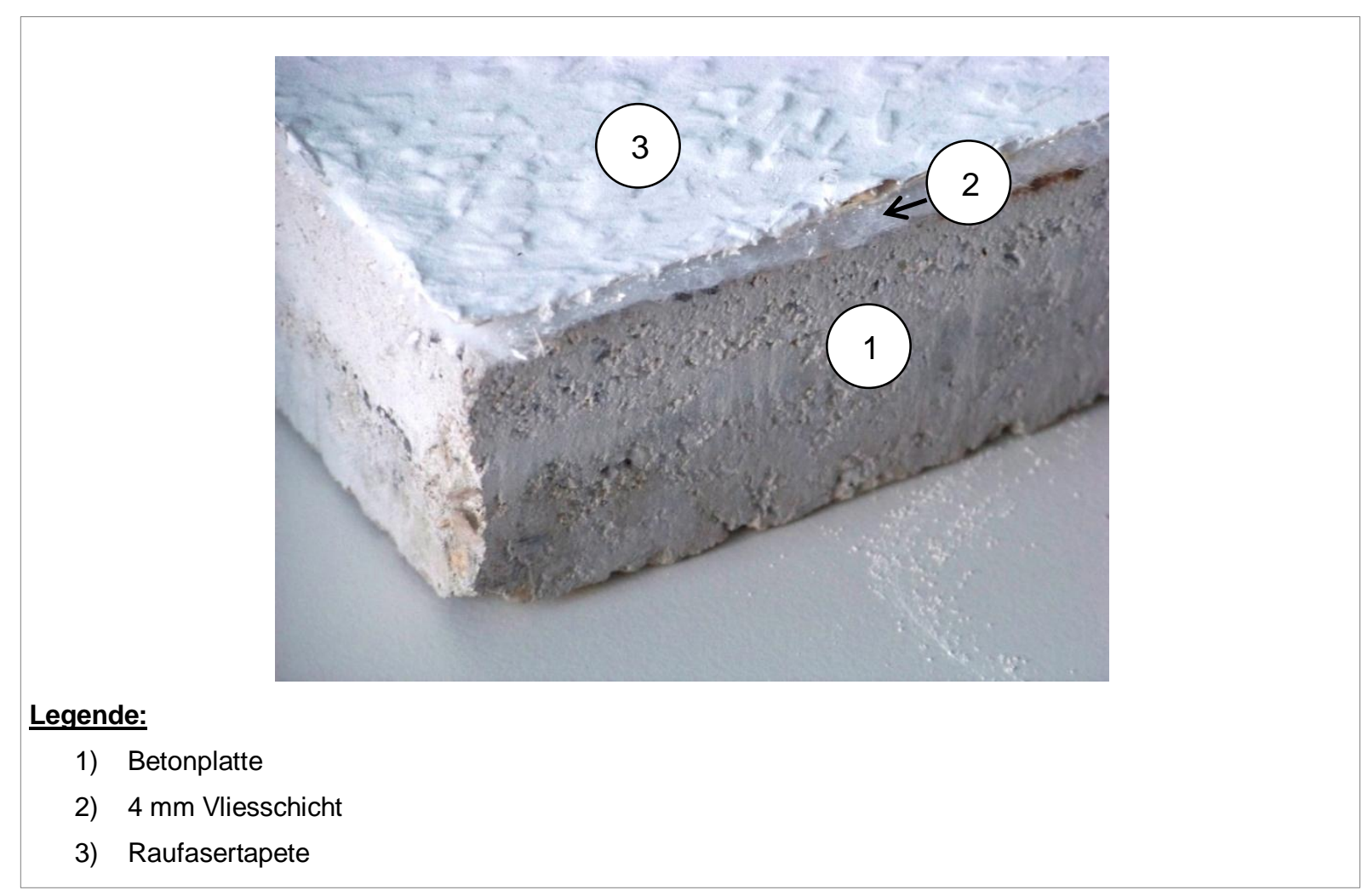

Abbildung 1.2: Betonplatte beschichtet mit einer $4 \mathrm{~mm}$ dicken Vliesschicht 


\section{Wärmetransport}

\subsection{Konvektion}

In Fluiden (Gasen und Flüssigkeiten) findet neben der Wärmeleitung und ein Wärmestrahlungsaustausch, Konvektion statt. Bei der Konvektion unterscheidet man zwischen der freien und der erzwungenen Konvektion. Die freie Konvektion hat ihre Ursache in Temperaturunterschieden. Im Gegensatz dazu wird das Medium bei der erzwungenen Konvektion durch Hilfsmittel wie Ventilatoren oder Pumpen bewegt.

Der Wärmeübergangskoeffizient ist bei der erzwungenen Konvektion um ein Vielfaches höher als bei der freien Konvektion, vergleiche Tabelle 2.1.

\begin{tabular}{|l|c|c|}
\hline Konvektion & Medium & Wärmeübergangskoeffizient $\mathbf{h}_{\mathbf{K}}$ in $\left[\frac{\boldsymbol{W}}{\boldsymbol{m}^{2} \cdot \boldsymbol{K}}\right]$ \\
\hline frei, d. h. hier lokal thermisch bedingt & Luft & 3 bis 10 \\
& Wasser & 100 bis 600 \\
\hline $\begin{array}{l}\text { erzwungen, maschinell bedingt oder durch globale } \\
\text { Strömungen verursacht }\end{array}$ & Luft & 10 bis 100 \\
\hline
\end{tabular}

Tabelle 2.1: Wärmeübergangskoeffizienten bei freier und erzwungener Konvektion [18]

Die erzwungene Konvektion wird zum Beispiel gezielt bei Warmluftheizungen eingesetzt. Hierdurch wird eine schnellere Wärmeverteilung in der Luft hergestellt. Die konvektive Wärmeübertragung zwischen einem Gas oder einer Flüssigkeit und einem festen Körper wird durch folgende Parameter beeinflusst:

- Temperaturdifferenz zwischen Fluid und Körperoberfläche

- Geschwindigkeit des Fluids

- Strömungsart: laminar oder turbulent

- Anströmungssituation

- Größe der wärmeübertagenden Fläche

- Rauigkeit der wärmeübertragenden Fläche

In der Praxis ist die Annahme eines Wärmeübergangskoeffizienten für die Berechnung mit Schwierigkeiten behaftet [19]. Luftströmungen und -geschwindigkeiten in Räumen werden entscheidend durch räumliche Gegebenheiten beeinflusst, wie zum Beispiel durch Einrichtungsgegenstände, Heizkörperanordnungen und Fensterflächen. 
Die allgemeine Gleichung zur Ermittlung der übertragenen Wärmeenergie (Wärmestromdichte) lautet.

$\boldsymbol{q}_{c}=\boldsymbol{h}_{c} \cdot\left(\boldsymbol{\theta}_{\boldsymbol{a}}-\boldsymbol{\theta}_{s i}\right)\left[\boldsymbol{W} \cdot \boldsymbol{m}^{-2}\right]$

Formel 2.1: Konvektionsgleichung zur Ermittlung der Wärmestromdichte

mit:

$\begin{array}{lll}\mathrm{q}_{\mathrm{c}} & {\left[\frac{\mathrm{W}}{\mathrm{m}^{2}}\right]} & \text { konvektiv übertragene Wärmestromdichte } \\ \mathrm{h}_{\mathrm{c}} & {\left[\frac{\mathrm{w}}{\mathrm{m}^{2} \cdot K}\right]} & \text { konvektiver Wärmeübergangskoeffizient } \\ \theta_{\mathrm{a}} & {\left[{ }^{\circ} \mathrm{C}\right]} & \text { Raumlufttemperatur } \\ \theta_{\mathrm{si}} & {\left[{ }^{\circ} \mathrm{C}\right]} & \text { Oberflächentemperatur }\end{array}$




\subsection{Wärmestrahlung}

Es findet ein Wärmetransport durch elektromagnetische Wellen - vorwiegend im Wellenlängenbereich zwischen 0,8 bis $400 \mu \mathrm{m}$ - statt. Für den Strahlungsaustausch ist keine Materie erforderlich und deshalb ist dieser Austausch auch im Vakuum gleichermaßen gegeben. Der wärmere Körper gibt durch elektromagnetische Wellen mehr Wärme als ein hierzu kälterer Körper ab. Jeder Körper gibt Wärmestrahlung ab, das heißt je höher die Temperatur des Körpers ist, umso mehr Strahlung wird abgegeben, wobei der Emissionsgrad mit zu berücksichtigen ist.

\begin{tabular}{|l|c|}
\hline Oberfläche & Emissionsgrad $\varepsilon[-]$ \\
\hline Beton & 0,93 \\
\hline Holz & 0,94 \\
\hline Putz & 0,93 \\
\hline Ziegelstein, rot & 0,93 \\
\hline Stahl, nicht oxidiert & 0,24 \\
\hline Stahl, oxidiert & 0,80 \\
\hline
\end{tabular}

Tabelle 2.2: Emissionsgrade verschiedener Baumaterial-Oberflächen [20]

Die elektromagnetischen Wellen werden entsprechend anteilig vom Körper reflektiert, durchgelassen und absorbiert, vergleiche Abbildung 2.1.

Vom Körper aufgenommene Wärmemenge:

$Q=E-A$

Formel 2.2: Absorbierte Wärmemenge durch Strahlungseinflüsse

mit:

$\begin{array}{lll}\text { Q } & {[W]} & \text { vom Körper aufgenommener Wärmestrom } \\ E & {[W]} & \text { vom Körper emittierte Wärmestrahlung } \\ \text { A } & {[W]} & \text { vom Körper absorbierte Wärme }\end{array}$




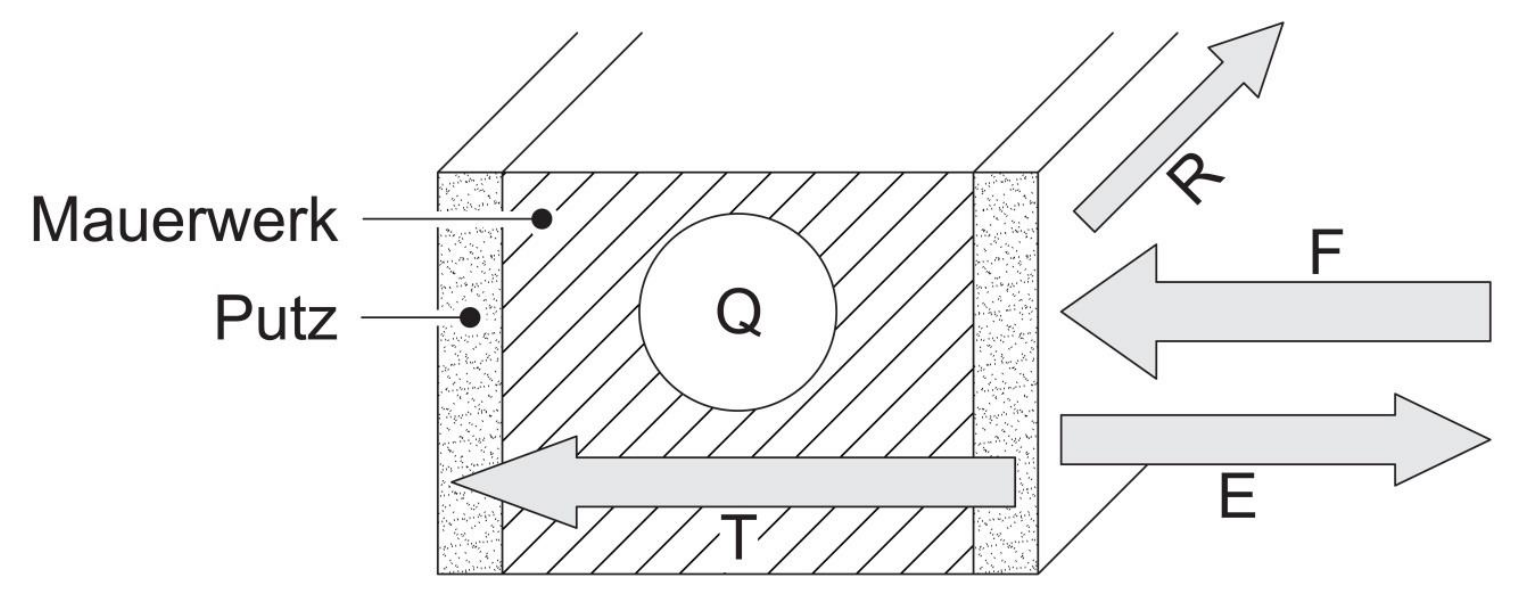

Abbildung 2.1: Energiebilanz beim Wärmetransport an und in einer Wand (für eine Wandseite betrachtet)

mit:

$\begin{array}{lll}\text { Q } & {[W]} & \text { von der Wand durch Wärmespeicherung aufgenommene Wärme } \\ \text { E } & {[W]} & \text { von der Wand emittierte Wärmestrahlung } \\ F & {[W]} & \text { empfangene Wärmeeinstrahlung an der Wandoberfläche } \\ \text { R } & {[W]} & \text { von der Wand reflektierte Wärmestrahlung } \\ \text { T } & {[W]} & \text { Transmissionswärmestrom durch die Wand }\end{array}$

Heizungssysteme, welche hauptsächlich die Wärme durch Wärmestrahlung abgeben, haben den Vorteil, dass die Luft weniger bewegt und damit weniger verschmutzt wird. Die angestrahlten Oberflächen werden dabei direkt erwärmt, wodurch eine schnelle Anheizgeschwindigkeit bei wenig Speichermasse erzielt wird. Ein Beispiel hierfür ist die Erwärmung in einem Zelt. 


\subsection{Stationäre Wärmeleitung}

Die Wärmeleitung beschreibt den Transport einer Wärmemenge innerhalb eines Stoffes von der wärmeren zur kälteren Seite. Bei einer stationären Betrachtung sind im Gegensatz zur instationären Wärmeleitung die jeweiligen Wärmespeicher dem Temperaturniveau entsprechend gefüllt (gesättigt), so dass die Wärmespeicherfähigkeit des Baustoffes in diesem Sonderfall keine Bedeutung hat und nur noch die Wärmeleitfähigkeit $\lambda$ (in $W /(m \cdot K)$ ) und die Schichtdicke berücksichtigt werden müssen.

Die Wärmeleitfähigkeit gibt die Wärmestromdichte (in W) pro Quadratmeter für eine $1 \mathrm{~m}$ dicke Schicht bei einer Temperaturdifferenz von 1 Kelvin an. Bei typischen mineralischen Baustoffen entscheidet neben der reinen Materialeigenschaft die Porengröße und -menge über die Wärmeleitfähigkeit. Durch die Porosität werden neben der Wärme gegebenenfalls auch Feuchtemengen transportiert. Mit dem Feuchtetransport, welcher tendenziell in Richtung Temperaturgefälle erfolgt, wird zusätzlich Wärme durch Verdunstung latent durch Diffusion und Kondensation transportiert (gegebenenfalls in einer maßgeblichen Menge).

Für einschichtige Bauteile gilt:

$Q=\frac{A \cdot t}{\frac{d}{\lambda}} \cdot\left(\theta_{1}-\theta_{2}\right)$

Formel 2.3: Wärmeleitung durch Transmission für ein einschichtiges Bauteil ohne Feuchtetransporteinfluss

Für mehrschichtige Bauteile gilt:

$Q=\frac{A \cdot t}{\sum \frac{d_{i}}{\lambda_{i}}} \cdot \Delta T$

Formel 2.4: Wärmeleitung durch Transmission für mehrschichtige Bauteile ohne Feuchtetransporteinfluss

mit:

$\begin{array}{lll}\mathrm{Q} & {[\mathrm{Ws}]} & \text { transportierte Wärmemenge } \\ \mathrm{A} & {\left[\mathrm{m}^{2}\right]} & \text { Querschnittsfläche, durch welche Wärme strömt } \\ \mathrm{t} & {[\mathrm{s}]} & \text { Zeit } \\ \theta & {\left[{ }^{\circ} \mathrm{C}\right]} & \text { Temperatur } \\ \lambda_{\mathrm{i}} & {\left[\frac{\mathrm{W}}{\mathrm{m} \cdot \mathrm{K}}\right]} & \text { Wärmeleitfähigkeit der jeweiligen Schicht } \\ \mathrm{d}_{\mathrm{i}} & {[\mathrm{m}]} & \text { Dicke der jeweiligen Schicht }\end{array}$




\subsection{Instationäre Wärmeleitung}

Außen- und Innenklima sind nicht konstant, so dass sich die Temperaturen auf beiden Seiten eines Außen- und Innenwandbauteils ständig verändern. Diese Veränderungen führen dazu, dass ein instationärer Wärmetransport stattfindet. Hierbei werden die jeweiligen Wärmespeicher immer wieder geladen und entladen, vergleiche Abbildung 2.2.

Bei einer genaueren Betrachtung des wärmetechnischen Verhaltens eines Bauteils kann der stationäre Zustand nur selten angenommen werden.

Bei der instationären Betrachtung ist neben der Wärmeleitfähigkeit $\lambda$ auch die Wärmespeicherfähigkeit in den jeweiligen Bauteilschichten von großer Bedeutung. Jede Schicht eines Bauteils speichert Wärmemengen, so dass neben der Wärmeleitung auch eine Wärmespeicherung in den jeweiligen Teilschichten stattfindet.

Übliche Wandkonstruktionen bestehen oft aus unterschiedlichen Bauteilschichten mit verschiedenen thermischen Eigenschaften, wobei jede Schicht die Eigenschaften Wärmeleitung und -speicherung aufweist. Stellt man wie in Abbildung 2.2 eine massive Innenwand schematisch dar, besteht diese im Wesentlichen aus nur drei Schichten.

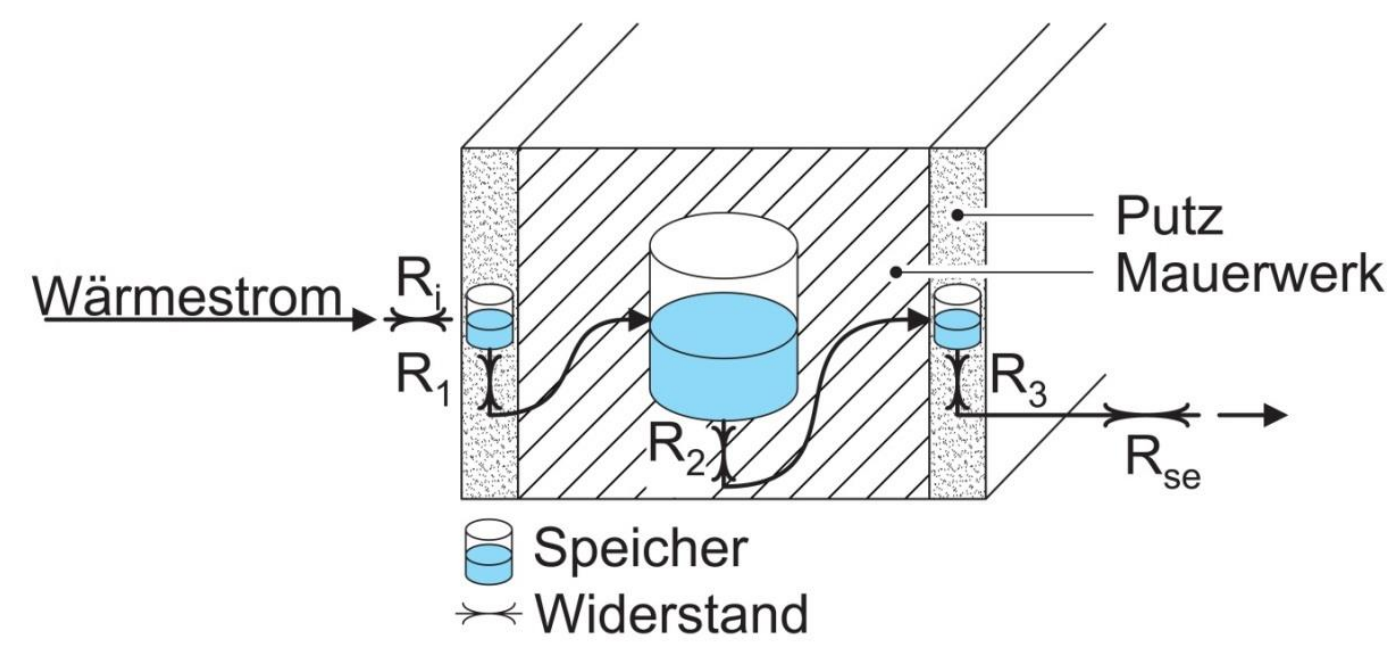

Abbildung 2.2: Schematische Darstellung der Wärmeleitung und -speicherung in einem einfachen Wandquerschnitt bei wechselndem Temperaturniveau

Bei einer instationären Betrachtung ergibt sich, dass neben den thermischen Stoffeigenschaften auch die Lage und Dicke der unterschiedlichen Schichten bei der instationären Betrachtung der Wärmeleitung für das thermische Verhalten der Konstruktion von Bedeutung ist. 
Die instationäre eindimensionale Wärmeleitung wird durch die partielle Differentialgleichung nach Fourier beschrieben mit:

$\frac{\delta \theta}{\delta t}=a \cdot \frac{\delta^{2} \theta}{\delta x^{2}}$

Formel 2.5: Differentialgleichung nach Fourier für die eindimensionale Wärmeübertragung

$a=\frac{\lambda}{c \cdot \rho}$

Formel 2.6: Gleichung zur Bestimmung des Temperaturleitkoeffizienten a

$\frac{\delta \theta}{\delta t}=\frac{\lambda}{c \cdot \rho} \cdot \frac{\delta^{2} \theta}{\delta x^{2}}$

Formel 2.7: Differentialgleichung nach Fourier mit den Stoffeigenschaften $\lambda, c$ und $\rho$

mit:

$\begin{array}{lll}\theta & {\left[{ }^{\circ} \mathrm{C}\right]} & \text { Temperatur } \\ \mathrm{t} & {[\mathrm{s}, \mathrm{h}]} & \text { Zeit } \\ \mathrm{x} & {[\mathrm{m}]} & \text { betrachtete Teilschichtdicke } \\ \mathrm{a} & {\left[\frac{\mathrm{m}^{2}}{\mathrm{~s}}\right]} & \text { Temperaturleitkoeffizient aus } \lambda, \mathrm{c} \text { und } \rho \text { des Stoffes } \\ \lambda & {\left[\frac{\mathrm{W}}{\mathrm{m} \cdot \mathrm{k}}\right]} & \text { Wärmeleitfähigkeit des Stoffes } \\ \mathrm{C} & {\left[\frac{\mathrm{kJ}}{\mathrm{kg} \cdot \mathrm{K}}\right]} & \text { spezifische Wärmekapazität auf die Masse bezogen } \\ \rho & {\left[\frac{\mathrm{kg}}{\mathrm{m}^{3}}\right]} & \text { Stoffdichte }\end{array}$


Die in Formel 2.5 dargestellte Differentialgleichung nach Fourier für den eindimensionalen Wärmetransport kann numerisch oder grafisch gelöst werden. Hierzu sind folgende Verfahren entwickelt worden. [21]:

- Grafische Lösung nach Binder/Schmidt

- Vorwärts-Differenzen-Verfahren

- Rückwärts-Differenzen-Verfahren

- Crank/Nicolson-Verfahren

Für die genannten Verfahren wird das Bauteil in Teilschichten aufgeteilt, wobei die Schichtaufteilung von der kleinsten zu betrachtenden Schicht und den betrachteten Zeitintervallen abhängig ist. In der Mitte der jeweils betrachteten Teilschicht wird ein Temperaturknotenpunkt angelegt. An jedem Temperaturknotenpunkt wird die Wärmebilanz mit den Materialparametern für die jeweilige Schicht unter Berücksichtigung der beiden Nachbarschichten ermittelt.

Die ersten Modellberechnungen in dieser Arbeit erfolgten mit dem Vorwärts-DifferenzenVerfahren unter Zuhilfenahme eines Tabellenkalkulationsprogramms. Durch die notwendigen Berechnungen für sehr kleine Teilschichten waren bei diesem Verfahren extrem viele Rechenschritte erforderlich. Im späteren Verlauf wurden die Berechnungen komfortabler mit Hilfe der in Kapitel 8 vorgestellten Software „HeatTransmission“ vorgenommen. 


\subsection{Der instationäre eingeschwungene Zustand}

Werden die Raumlufttemperaturen periodisch - innerhalb von 24 Stunden - verändert, so benötigt ein Bauteil einen längeren Zeitraum (einige Tage) bis sich ein regelmäßig zeitliches Temperaturprofil - beispielsweise auf der Wandoberfläche - ergibt.

In der Praxis ist eine periodische Temperaturveränderung der Raumlufttemperatur schon dadurch gegeben, dass eine Nachtabsenkung bei der Beheizung erfolgt, vergleiche Abbildung 2.3.

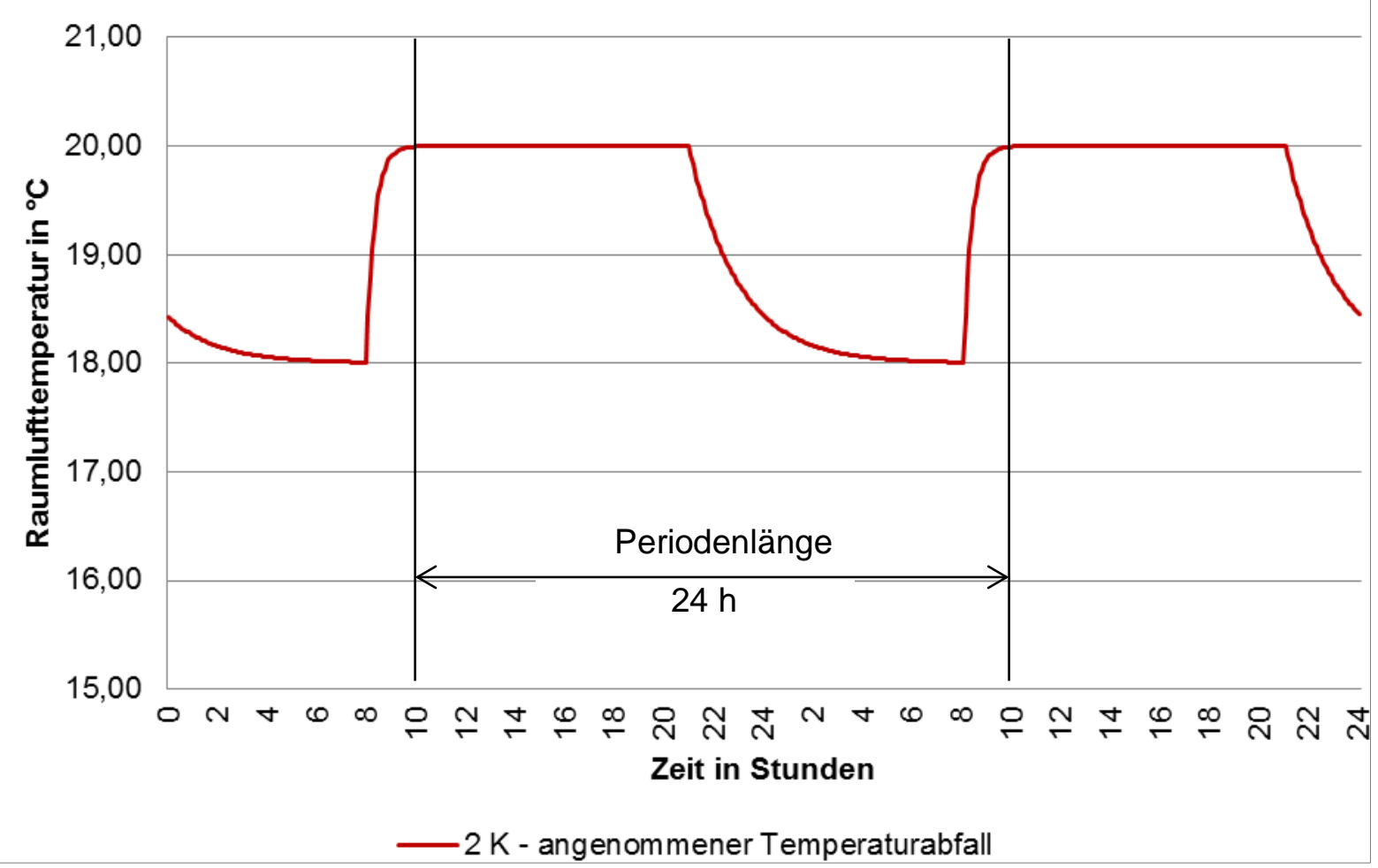

Abbildung 2.3: Raumlufttemperaturverlauf bei einer Nachtabsenkung um 2 Kelvin (K)

Der in Abbildung 2.3 dargestellte Temperaturverlauf hat eine Periodenlänge von 24 Stunden. Das bedeutet, dass nach jeweils 24 Stunden die gleiche Raumlufttemperatur gemessen wird. Die Temperaturen an der Oberfläche und im inneren eines Bauteils sind somit im eingeschwungenen Zustand nach jeweils 24 Stunden wieder identisch. 


\section{Wärmespeicherfähigkeit}

\subsection{Baustoffeigenschaften}

Das Wärmespeichervermögen von Baustoffen kann bei einer intermittierenden Beheizung von großer Bedeutung sein, weil die Wärmespeicherung Temperaturschwankungen bedämpft. Massivbauten können auf diese Weise im Sommer durch ihre Wärmespeicherfähigkeit einer zeitweiligen Übertemperierung entgegenwirken.

Für die Betrachtung der Wärmespeicherung eines Raumes ist die Masse sämtlicher Bauteile, welche den Raum umschließen, von Bedeutung. Der Speichermassenanteil der Außenbauteile ist oftmals von geringerer Bedeutung, da die massiven Außenwandflächen in der Regel recht klein in Relation zu den Innenbauteilflächen sind [22].

Durch die Wärmespeicherfähigkeit von Massivbauten ist in Räumen eine hinreichende bis gute Behaglichkeit, auch bei einer groben Regelungstechnik, möglich, vergleiche [7]. In den Übergangszeiten kann zudem solare Energie, welche tagsüber eingestrahlt wird, für die Energiebilanz nützlich gespeichert werden. Durch die gespeicherte Wärmeenergie erfolgt die Raumauskühlung bei niedrigeren Außentemperaturen langsamer.

Bei einer Beheizung mittels Einzelofen ist die Wärmespeicherung der Bauteile im Raum von noch größerer Bedeutung als bei einem geregelten Zentralbeheizungssystem, vergleiche [7]. Bei einem ungeregelten Beheizungssystem glätten die Speichermassen die Raumtemperaturschwankungen und es wird überschüssige Wärmeenergie entsprechend zwischengespeichert.

„Tritt keinerlei Überheizung auf und wird eine Nachtabsenkung betrieben, dann verbraucht eine Leichtbauweise weniger Energie als eine Schwerbauweise. Wird dagegen keine Nachtabsenkung durchgeführt, ist die Schwerbauweise günstiger, sobald Überheizungen auftreten." [23]

Feste, flüssige und gasförmige Stoffe können Wärmemengen speichern. Die „Speichergröße" ist stoffabhängig und kann mittels eines Kalorimeters durch Beobachtung der Temperaturveränderung eines entsprechend temperierten Körpers in einem praktisch thermisch geschlossenen System bestimmt werden. Mit einem Kalorimeter kann die Temperaturänderung gemessen und daraus die spezifische Wärmekapazität eines Stoffes ermittelt werden. 
Die spezifische Wärmekapazität $c$ ist die Wärmemenge, die nötig ist, um 1 Kilogramm eines Stoffes um 1 Kelvin zu erwärmen.

$c=\frac{\Delta Q}{m \cdot \Delta T}$

Formel 3.1: Spezifische Wärmekapazität c

mit:

$\begin{array}{lll}\mathrm{c} & {\left[\frac{\mathrm{J}}{\mathrm{kg} \cdot \mathrm{K}}\right]} & \text { spezifische Wärmekapazität } \\ \mathrm{Q} & {[\mathrm{J}]} & \text { Wärmemenge } \\ \mathrm{m} & {[\mathrm{kg}]} & \text { Masse des Stoffes } \\ \mathrm{T} & {[\mathrm{K}]} & \text { absolute Temperatur }\end{array}$

Stellt man die Formel 3.1 nach $\Delta Q$ um, ergibt sich hieraus eine gespeicherte Wärmemenge für eine entsprechende Temperaturveränderung:

$\Delta Q=m * c * \Delta T$

Formel 3.2: Wärmemenge abhängig vom Stoff und einer Temperaturdifferenz vor und nach der Wärmeaufnahme oder -abgabe

Für den praktischen Vergleich ist die Benennung der volumenbezogenen Wärmespeicherkapazität sinnvoll. Diese wird durch den Wärmespeicherwert $S$ dargestellt.

$S=\rho * C$

Formel 3.3: Wärmespeicherwert S

mit:

$\begin{array}{lll}\text { S } & {\left[\frac{\mathrm{J}}{\mathrm{m}^{3} \cdot \mathrm{K}}\right]} & \text { Wärmespeicherwert } \\ \rho & {\left[\frac{\mathrm{kg}}{\mathrm{m}^{3}}\right]} & \text { Stoff-Rohdichte } \\ \text { C } & {\left[\frac{\mathrm{J}}{\mathrm{kg} \cdot \mathrm{K}}\right]} & \text { spezifische Wärmekapazität }\end{array}$


Stellt man die spezifische Wärmekapazität c und den Wärmespeicherwert typischer Baustoffe grafisch gegenüber, wird der Unterschied zwischen dem masse- bzw. volumenbezogenen Speicherverhalten deutlich, weil die Rohdichten sehr unterschiedlich sind. Bei den meisten Baustoffen liegt die spezifische Wärmekapazität nahe beieinander. Bei mineralischen Baustoffen ist die spezifische Wärmekapazität (fast) gleich groß ( 1000 $\left.\left[\frac{\mathrm{J}}{\mathrm{kg} \cdot \mathrm{K}}\right]\right)$.

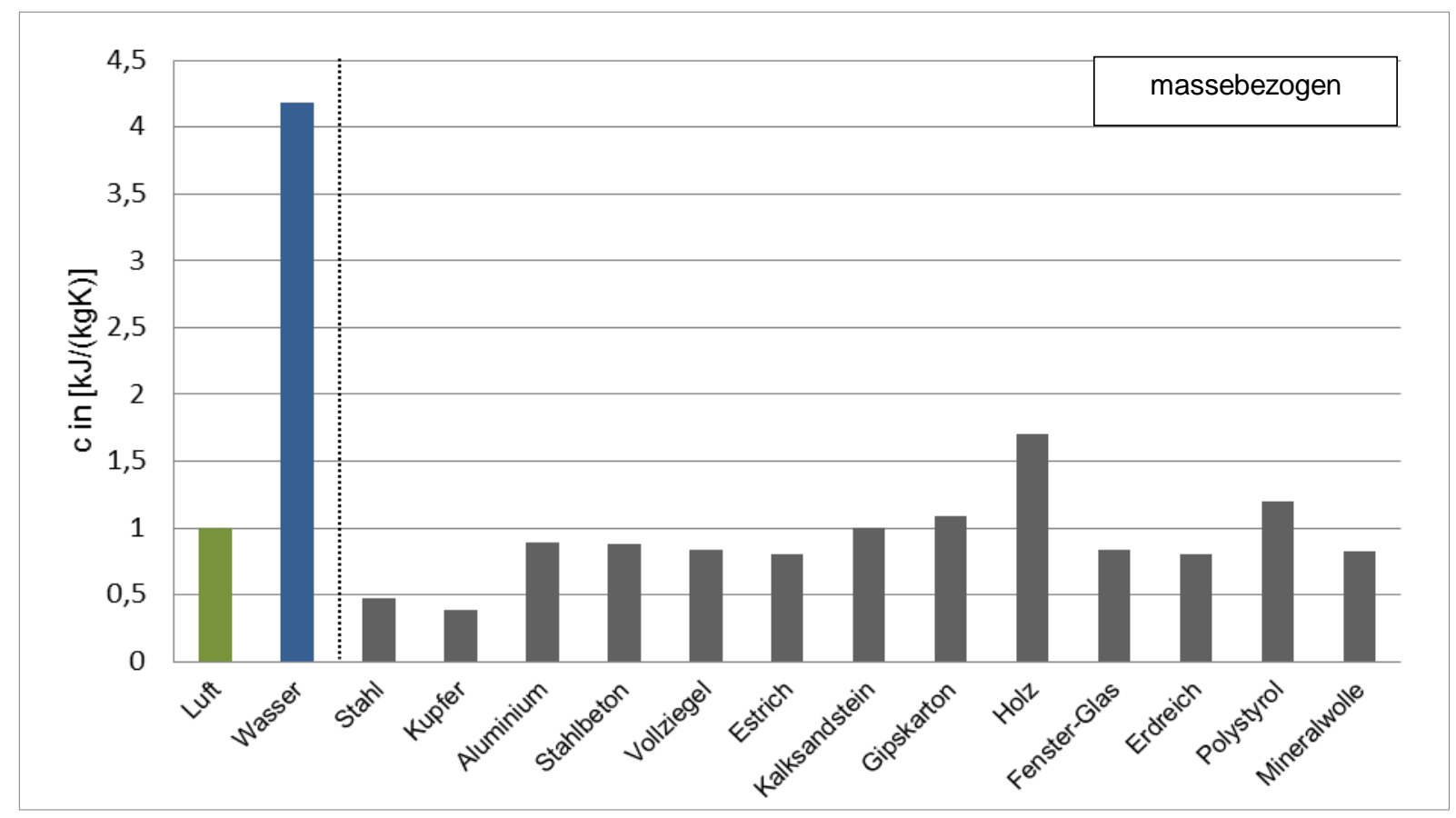

Abbildung 3.1: Spezifische Wärmekapazität unterschiedlicher Baustoffe [24]

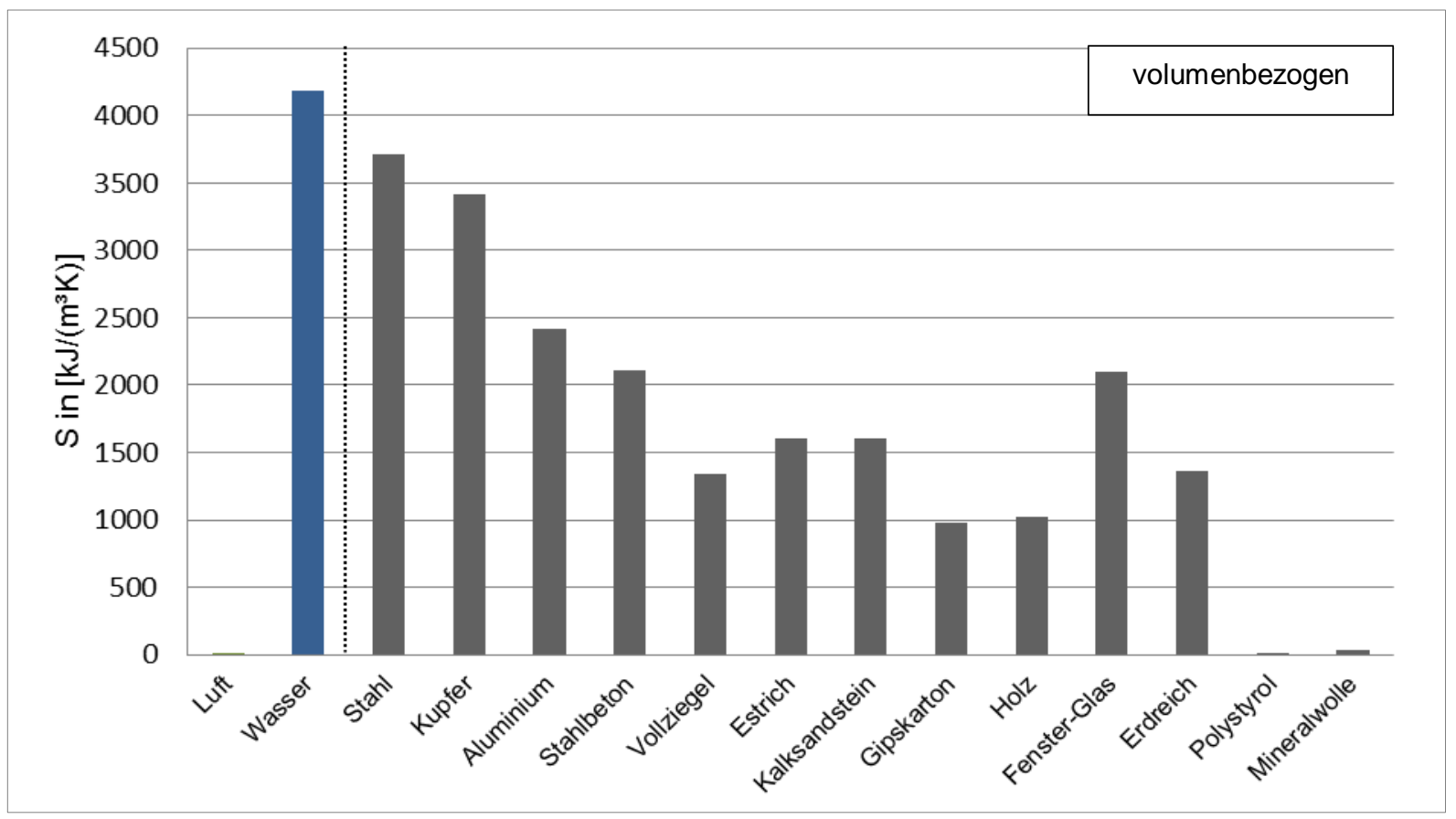

Abbildung 3.2: Wärmespeicherwert unterschiedlicher Baustoffe [24] [25] 
Aus den Abbildungen 3.1 und 3.2 geht deutlich hervor, dass Luft eine sehr geringe volumenbezogene Wärmespeicherfähigkeit aufweist. Die Baustoffe, welche üblicherweise verwendet werden, weisen demgegenüber eine vielfach größere volumenbezogene Wärmespeicherfähigkeit auf (durch die großen Rohdichteunterschiede $\rho_{\text {Luft }}=1,2 \mathrm{~kg} / \mathrm{m}^{3}$ und $\rho_{\text {Baustoffe }} z$ wischen $30 \ldots 2500\left[\frac{\mathrm{kg}}{\mathrm{m}^{3}}\right]$. Aus Abbildung 3.2 wird deutlich, dass die Baustoffwahl den Energieaufwand bei einer Raumanheizung maßgeblich beeinflussen kann. 


\subsection{Bauteileigenschaften unter instationären Randbedingungen}

\subsubsection{Thermische Eindringtiefe}

Der eingeschwungene Zustand ist durch einen regelmäßig wiederkehrenden Temperaturverlauf gekennzeichnet, vergleiche Kapitel 2.5 dieser Arbeit. Beim eingeschwungenen Zustand werden die Bauteilrandbereiche thermisch stärker beeinflusst, während die inneren Bauteilschichten können nahezu konstante mittlere Temperatur aufweisen.

Die thermische Eindringtiefe gibt an, wie groß der Bauteilrandbereich ist, in welchem sich das Temperaturprofil ständig verändert. Die Eindringtiefe hängt von der angrenzenden Raumlufttemperaturänderung und den Baustoffeigenschaften der Wandkonstruktion ab. Für die Praxis bedeutet dies, dass bei einer Anheizung vorwiegend in diesem Bauteilbereich Energie gespeichert wird und in der Auskühlphase wieder Energie abgegeben wird.

Durch eine Dämmschicht in oder an der Wandkonstruktion kann das Eindringen der Temperaturwelle gebremst werden, so dass eine Dämmstoffschicht die Eindringtiefe deutlich reduziert.

Es zeigt sich, wie in Kapitel 8.4 dargestellt, dass die Temperaturwelle bei einer üblichen nächtlichen Temperaturabsenkung durch die gesamte Wandkonstruktion geht. Nur bei kleineren Heizungsunterbrechungen kann die Eindringtiefe allein bezogen auf den Randbereich der Wandkonstruktion betrachtet werden.

Somit muss in der Regel die Eindringtiefe bis zur nächstfolgenden Dämmschichtebene betrachtet werden. Bei einer Innenwand kann die Eindringtiefe bis zur Hälfte der Wand angesetzt werden, wenn der benachbarte Raum die gleiche Temperaturentwicklung aufweist [26]. 


\subsubsection{Wirksame Wärmespeicherfähigkeit}

Die wirksame Speicherfähigkeit beschreibt die Speicherfähigkeit eines Bauteils im oberflächennahen Bereich eines raumumschließenden Bauteils.

$c_{\text {wirk }}=\sum_{i=1}^{n} \rho_{i} \cdot c_{i} \cdot d_{i} \cdot A_{i}$

Formel 3.4: Wirksame Speicherfähigkeit von raumumschließenden Bauteilen

mit:

$\begin{array}{lll}\mathrm{c}_{\text {wirk }} & {\left[\frac{\mathrm{Wh}}{\mathrm{K}}\right]} & \text { wirksame Speicherfähigkeit eines Raumes } \\ \rho_{\mathrm{i}} & {\left[\frac{\mathrm{kg}}{\mathrm{m}^{3}}\right]} & \text { Dichte des Materials i } \\ \mathrm{c}_{\mathrm{i}} & {\left[\frac{\mathrm{Wh}}{\mathrm{kg} \cdot \mathrm{K}}\right]} & \text { spezifische Wärmespeicherfähigkeit des Materials i } \\ \mathrm{d}_{\mathrm{i}} & {[\mathrm{m}]} & \text { Dicke des Materials i } \\ \mathrm{A}_{\mathrm{i}} & {\left[\mathrm{m}^{2}\right]} & \text { Fläche des Bauteils i }\end{array}$

Die hier definierte wirksame Speicherfähigkeit ist bei den in dieser Arbeit vorgenommenen Untersuchungen nur bedingt verwendbar, weil die untersuchten Dämmstoffschichten an den raumseitigen Wandflächen vorwiegend sehr dünn sind und demnach eine Wechselwirkung mit dem darunterliegenden Wärmespeicher erfolgt. Hier muss durch genauere Untersuchungen, wie diese in Kapitel 8.4 dargestellt sind, das Speicherverhalten der Wand ermittelt werden. 


\section{Thermische Behaglichkeit in Innenräumen}

\subsection{Kriterien}

Das thermische Raumklima beeinflusst die Wohn- und Arbeitsqualität maßgeblich. Folgende messbare Größen sind für die thermische Behaglichkeit eines Raumes bedeutend:

- Raumlufttemperatur

- Temperatur der Bauteiloberflächen

- Luftgeschwindigkeit (Zuglufteinwirkungen)

- Luftfeuchtigkeit

Diese Faktoren sind bei der Gebäudeplanung oder -renovierung entsprechend der Raumnutzung zu berücksichtigen. In der vorliegenden Arbeit werden die Raumlufttemperaturen und insbesondere die Wandteiloberflächentemperaturen betrachtet.

\subsection{Thermische Behaglichkeit in Innenräumen}

Für gute Aufenthaltsbedingungen in Innenräumen ist die thermische Behaglichkeit ein entscheidender Faktor. Sie beschreibt ein wichtiges Qualitätskriterium für Wohn- und Arbeitsräume, weil die Behaglichkeit für die Leistungsfähigkeit und Gesundheit von großer Bedeutung ist. Bei einem hohen Behaglichkeitsgrad ist die Leistungsfähigkeit und -bereitschaft entsprechend hoch [27] [28] [29] [30]. Nach Peters, Zerwas, Kempen und Krause [31] kann die Verbesserung der Behaglichkeit in Arbeitsräumen zu einer signifikanten Arbeitsleistungssteigerung führen, so dass sich die Investitionskosten für Verbesserungen leicht amortisieren.

\footnotetext{
„Das Wohlbefinden eines Menschen wird hauptsächlich im Unterbewusstsein verarbeitet und oftmals unterschätzt. Ins Bewusstsein rückt es meist erst, wenn die Unbehaglichkeit stark ausgeprägt ist. Die daraus resultierenden Folgen sind unterschiedlich und wirken sich negativ auf die Leistung aus, z.B. sinkt die Leistungsfähigkeit bei hohen Temperaturen im Sommer, ein Mitarbeiter wird krank oder wechselt die Stelle. Die Folgen für das Unternehmen sind erhöhte Kosten durch Umsatzreduzierung, Arbeitsausfall oder die Einarbeitung neuer Mitarbeiter." [31]
}

Die Leistungsanforderungen haben sich insbesondere durch moderne und hocheffiziente Kommunikationsmittel, welche eine schnellere Kommunikation und eine größere Zahl an Kommunikationsprozessen ermöglichen, erhöht. 
Die Folge ist leider auch eine wachsende Anzahl an stressbedingten Krankheitsausfällen, die auch durch das „Sickbuilding-Syndrom“ bekannt geworden sind. Die Behaglichkeit in Arbeitsund Wohnräumen muss schon allein aus diesem Grund genauer betrachtet werden. Ziel sollte es sein, Stressfaktoren, welche das Nervensystem zusätzlich belasten, zu mindern.

Zudem ist bei der wirtschaftlichen Entwicklung in unserem Kulturkreis ein stetig steigender Komfortanspruch festzustellen. Im Wohnungs- und Bürobau werden immer hochwertigere Materialien und Konstruktion verwendet, welche die Baukosten und damit die Erwartungshaltung steigern. Neben dem optischen Erscheinungsbild wachsen die Anforderungen an die raumklimatische Qualität, welche durch die Empfindungstemperatur, die Raumluftfeuchte und Luftqualität gekennzeichnet ist. Der raumklimatische Toleranzbereich ist gegenüber vorhergehenden Generationen deutlich kleiner geworden. Kleinste Störwirkungen werden oft schon beanstandet. Dies beruht zum Beispiel auf „Ballungsstress“, „Erwartungshaltungen“ und veränderten Bauweisen, welche unter anderem durch offene Grundrisse, geringe Grundgeräusche durch dichtere Fensterkonstruktionen und Ähnliches verursacht werden.

Die vorliegende Arbeit beschränkt sich auf eine Untersuchung und Bewertung der Raumluftund Oberflächentemperaturen, wobei die thermische Behaglichkeit insgesamt von folgenden messbaren physikalischen Größen abhängt:

- Raumlufttemperatur

- mittlere Strahlungstemperatur

- Luftbewegung

- relative Luftfeuchte

Die Messung der physikalischen Größen ist in DIN EN ISO 7726 [32] beschrieben. Neben diesen Werten sind weitere Faktoren von Bedeutung. Eine Bewertung der Behaglichkeit ist auch von der jeweiligen Person sowie ihrer körperlichen Tätigkeit und Kleidung abhängig. Hieraus ergibt sich eine recht gut kalkulierbare Abhängigkeit zwischen der Innenraumtemperatur und den Anforderungen bei verschiedenen Raumnutzungen. 
Eine genauere Betrachtung kann mit der Komfort-Gleichung nach DIN EN ISO 7730 [33] erfolgen. Der thermische Behaglichkeitskorridor für Innenräume ist sehr eng und wird maßgeblich aus den Temperaturen der Raumluft und der Oberflächen bestimmt.

Die maßgebliche Wirktemperatur, auch operative Temperatur oder früher Empfindungstemperatur genannt, ist ein Mittelwert aus der Lufttemperatur und den gemittelten Oberflächentemperaturen im Raum, weil eine Person im Normalfall die Wärme etwa in gleicher Größenordnung durch Strahlung und Konvektion abgibt. Die operative Temperatur kann aus der Lufttemperatur und der mittleren Strahlungstemperatur der Oberflächen gebildet werden.

Operative Temperatur:

$\theta_{O}=\frac{\theta_{a}+\theta_{r}}{2}$

Formel 4.1: Mittelungsgleichung zur Bestimmung der operativen Temperatur

mit

$\begin{array}{lll}\theta_{\circ} & {\left[{ }^{\circ} \mathrm{C}\right]} & \text { operative Temperatur } \\ \theta_{\mathrm{a}} & {\left[{ }^{\circ} \mathrm{C}\right]} & \text { Raumlufttemperatur } \\ \theta_{\mathrm{r}} & {\left[{ }^{\circ} \mathrm{C}\right]} & \text { mittlere Oberflächentemperatur }\end{array}$

Mittlere Strahlungstemperatur:

$\theta_{r}=\frac{\sum A_{i} \cdot \theta_{r}}{\sum A_{i}}$

Formel 4.2: Mittlere Strahlungstemperatur der raumumschließenden Oberflächen

mit

$\begin{array}{lll}\theta_{\mathrm{r}} & {\left[{ }^{\circ} \mathrm{C}\right]} & \text { mittlere Oberflächentemperatur im Raum }\end{array}$

$\mathrm{A}_{\mathrm{i}} \quad\left[\mathrm{m}^{2}\right] \quad$ Telfläche der raumumschließenden Oberflächen

Die mittlere Oberflächentemperatur setzt sich aus den raumumschließenden Bauteilen Wand, Boden und Decke zusammen. Diese vereinfachte Betrachtung ist bei einer lokalen Besonderheit im Raum nicht ausreichend, da beispielsweise durch große Abweichungen bei den Strahlungstemperaturen ein asymmetrisches Temperaturfeld auf Personen einwirken kann, welches zu einer größeren Belastung führt, vergleiche DIN EN ISO 7730 [33]. 
Die Personen-Bekleidung wird mit dem Bekleidungswert (clo) berücksichtigt. Die clo-Werte wurden an stehenden Menschen gemessen.

\begin{tabular}{|l|c|c|}
\hline Bekleidungsart & clo & {$\left[\frac{\boldsymbol{m}^{2} \cdot \boldsymbol{K}}{\boldsymbol{W}}\right]$} \\
\hline Unbekleidet & 0 & 0 \\
\hline Turnbekleidung / Tropenbekleidung & 0,3 bis 0,4 & 0,05 bis 0,06 \\
\hline Leichte Sommerbekleidung & 0,5 & 0,078 \\
\hline Leichte Arbeitsbekleidung & 0,6 bis 0,7 & 0,11 \\
\hline Feste Arbeitskleider / leichter Straßenanzug & 1,0 & 0,233 \\
\hline Traditionelle europäische Stadtbekleidung & 1,5 & 0,32 \\
\hline Kleidung für nasskaltes Wetter & 2,0 & 0,16 \\
\hline
\end{tabular}

Tabelle 4.1: Bekleidungsfaktor nach EN ISO 7730:2005 [34]

Die Aktivität einer Person und der daraus resultierende Energieumsatz wird in met angegeben, 1 met $=58 \mathrm{~W} / \mathrm{m}^{2}$. Die Oberfläche einer Person ist mit circa $2 \mathrm{~m}^{2}$ anzunehmen.

\begin{tabular}{|l|c|c|}
\hline \multirow{2}{*}{ Aktivität } & \multicolumn{2}{|c|}{ Energieumsatz } \\
\cline { 2 - 3 } & {$\left[\frac{W}{\mathbf{m}^{2}}\right]$} & met \\
\hline Angelehnt & 46 & 0,8 \\
\hline Sitzend, entspannt & 58 & 1,0 \\
\hline Stizende Tätigkeit & 70 & 1,2 \\
\hline Stehende, leichte Tätigkeit & 93 & 1,6 \\
\hline Stehende, mittelschwere Tätigkeit & 116 & 2,0 \\
\hline Gehen auf der Ebene: & & \\
& & 1,9 \\
$3 \mathrm{~km} / \mathrm{h}$ & 110 & 2,4 \\
$4 \mathrm{~km} / \mathrm{h} / \mathrm{h}$ & 140 & 2,8 \\
$5 \mathrm{~km} / \mathrm{h}$ & 165 & 3,4 \\
\hline
\end{tabular}

Tabelle 4.2: Aktivität und Energieumsatz von Personen nach EN ISO 7730:2005 [35] 
In DIN EN 12831 [36] sind Norm-Innenraumtemperaturen abhängig von der Raumnutzung, der Kleidung und Aktivität festgelegt, vergleiche Tabelle 4.3.

\begin{tabular}{|c|c|c|c|c|}
\hline $\begin{array}{c}\text { Gebäudeart / } \\
\text { Raum }\end{array}$ & $\begin{array}{c}\text { Bekleidung, Winter } \\
\text { clo }\end{array}$ & $\begin{array}{l}\text { Aktivität } \\
\text { met }\end{array}$ & $\begin{array}{l}\text { Klasse der Qualität der } \\
\text { Raumluftinnen- } \\
\text { temperatur }\end{array}$ & $\begin{array}{c}\text { Operative Soll- } \\
\text { Temperaturen in }{ }^{\circ} \mathrm{C}, \\
\text { Winter }\end{array}$ \\
\hline \multirow{3}{*}{$\begin{array}{l}\text { Einzelbüro } \\
\text { Großraumbüro } \\
\text { Konferenzraum } \\
\text { Auditorium } \\
\text { Cafeteria } \\
\text { Restaurant } \\
\text { Klassenraum } \\
\text { Kindergarten } \\
\text { Wohnungen }\end{array}$} & \multirow{3}{*}{1,0} & \multirow{3}{*}{1,2} & A & $21-23$ \\
\hline & & & B & $20-24$ \\
\hline & & & C & $19-25$ \\
\hline \multirow{2}{*}{$\begin{array}{l}\text { Kaufhaus } \\
\text { Museum } \\
\text { Galerie }\end{array}$} & \multirow[b]{2}{*}{1,0} & \multirow[b]{2}{*}{1,6} & A & $17,5-20,5$ \\
\hline & & & $B$ & $\begin{array}{l}16-22 \\
15-23\end{array}$ \\
\hline \multirow{3}{*}{ Badezimmer } & \multirow{3}{*}{0,2} & \multirow{3}{*}{1,6} & A & $24,5-25,5$ \\
\hline & & & $B$ & $23,5-26,5$ \\
\hline & & & C & $23-27$ \\
\hline \multirow{3}{*}{ Kirchen } & \multirow{3}{*}{1,5} & \multirow{3}{*}{1,3} & A & $16,5-19,5$ \\
\hline & & & B & $15-21$ \\
\hline & & & $\mathrm{C}$ & $14-22$ \\
\hline
\end{tabular}

Tabelle 4.3: Norm-Innentemperaturen nach DIN 21831 [36], abhängig von der Nutzung und dem Qualitätsanspruch

Die Klassen A, B und C geben den Prozentsatz unzufriedener Personen (Predicted Percentage of Dissatisfied-PPD) an.

\begin{tabular}{|l|l|}
\hline Klasse & PPD \\
\hline A & $<6 \%$ \\
\hline B & $<10 \%$ \\
\hline C & $<15 \%$ \\
\hline
\end{tabular}

Tabelle 4.4: Qualitätsklassen zur Bewertung des Raumklimas nach DIN EN 7730 [33] 


\subsection{Empirische Bewertungsgrundlagen}

Eine allgemeine Bewertung hat sich aus größeren Gruppen von Probanden ermitteln lassen. In der ISO 7730 wird eine statistische Bewertung (nach Fanger) zur Beurteilung der Behaglichkeit beschrieben.

Der Perdicted Mean Vote (PMV) stellt die Grundlage der Bewertung des thermischen Raumklimas gemäß ISO 7730 [33] dar. Der PMV bewertet das thermische Raumklima mit einer siebenstufigen Klimabeurteilungsskala [33].

\begin{tabular}{|c|c|}
\hline \multicolumn{2}{|c|}{ Siebenstufige Klimabeurteilungsskala } \\
\hline+3 & heiß \\
\hline+2 & warm \\
\hline+1 & etwas warm \\
\hline 0 & neutral \\
\hline-1 & etwas kühl \\
\hline-2 & kühl \\
\hline-3 & kalt \\
\hline
\end{tabular}

Tabelle 4.5: Siebenstufige Klimabeurteilungsskala nach ISO 7730 [33]

Eine gute thermische Behaglichkeit ist hiernach bei PMV $=0$ gegeben und wird mit "neutral“ bezeichnet. Bei diesem Wert ist quasi eine günstige Wärmeabgabebilanz zwischen dem menschlichen Körper und der Umgebung vorhanden. Eine gewisse Wärmemenge ist vom menschlichen Körper abzugeben, weil die Körpertemperatur sehr konstant gehalten werden muss.

Es kann jedoch auch bei einem PMV = 0 „neutral“ nicht davon ausgegangen werden, dass sämtliche Personen das Raumklima als angenehm bewerten. In der Praxis ist dieses Problem oftmals in Großraumbüros anzutreffen. Das Raumklima wird in solchen Räumen von einer großen Anzahl von Personen, jedoch nicht von allen, als angenehm empfunden. Es ist jedoch schwierig, hier ein für alle Personen passendes Raumklima herzustellen, da unterschiedliche Tätigkeiten und Bekleidungen sowie auch unterschiedliche „Klimazonen“ (Beispielsweise an Türen oder Fenstern) gegeben sind.

Um eine quantitative Aussage über die Bewertung des Raumklimas einer Gruppe von Personen zu erhalten, wird die zu erwartende Anzahl unzufriedener Personen PPD beziffert, vergleiche DIN EN ISO 7730 [33]. 
Der Zusammenhang zwischen PPD und den PMV wird in [33] mit folgender Näherungsgleichung beschrieben:

$p p d=100-95 * e^{-0,03353 * p m v^{4}-0,2179 * p m v^{2}}$

Formel 4.3: Zusammenhang zwischen PPD und PMV nach [32]

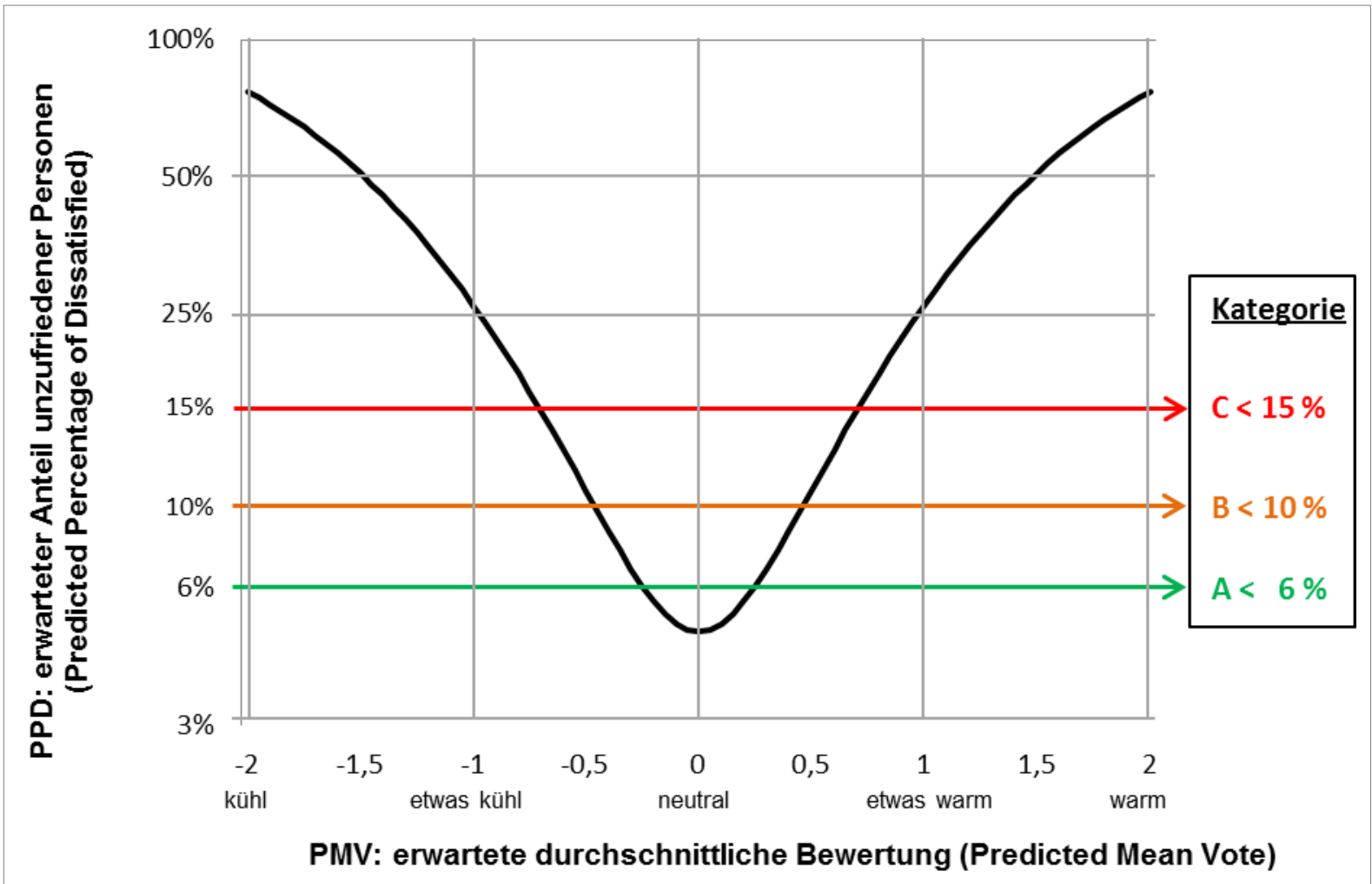

Abbildung 4.1: Zusammenhang zwischen erwarteter durchschnittlicher Bewertung des thermischen Komforts (PMV) und entsprechendem Prozentsatz unzufriedener Personen (PPD) 


\section{Beheizung}

\subsection{Nutzerverhalten}

Die sinnvolle Beheizung eines Gebäudes hängt von dessen Nutzung ab. Hierbei unterscheidet man grob zwischen Wohn- und Gewerbenutzung. Derartige Nutzungen führen zu typischen Heizungsunterbrechungen.

„Die Wirkung der Nachtabsenkung hängt von der Dauer der Heizungsunterbrechung ab. Im Wohnungsbau kommen hier meist 8 Stunden, im Bürobau bis etwa 14 Stunden in Frage." [23]

Bei der Wohnnutzung ist ein Zusammenhang zwischen den Beheizungszeiten und der Haushaltsnutzerzusammensetzung zu erkennen.

Je mehr Nutzer in einem Haushalt leben, um so kontinuierlicher werden in der Regel die Nutzungs- und Beheizungszeiten. In einem Single-Haushalt wird die Nutzung von einer Person bestimmt und ist abhängig von deren beruflichem Einsatz. Es gibt typische Nutzungsfälle, welche in Abbildung 5.1 in Form von schematischen Beheizungssituationen dargestellt sind.

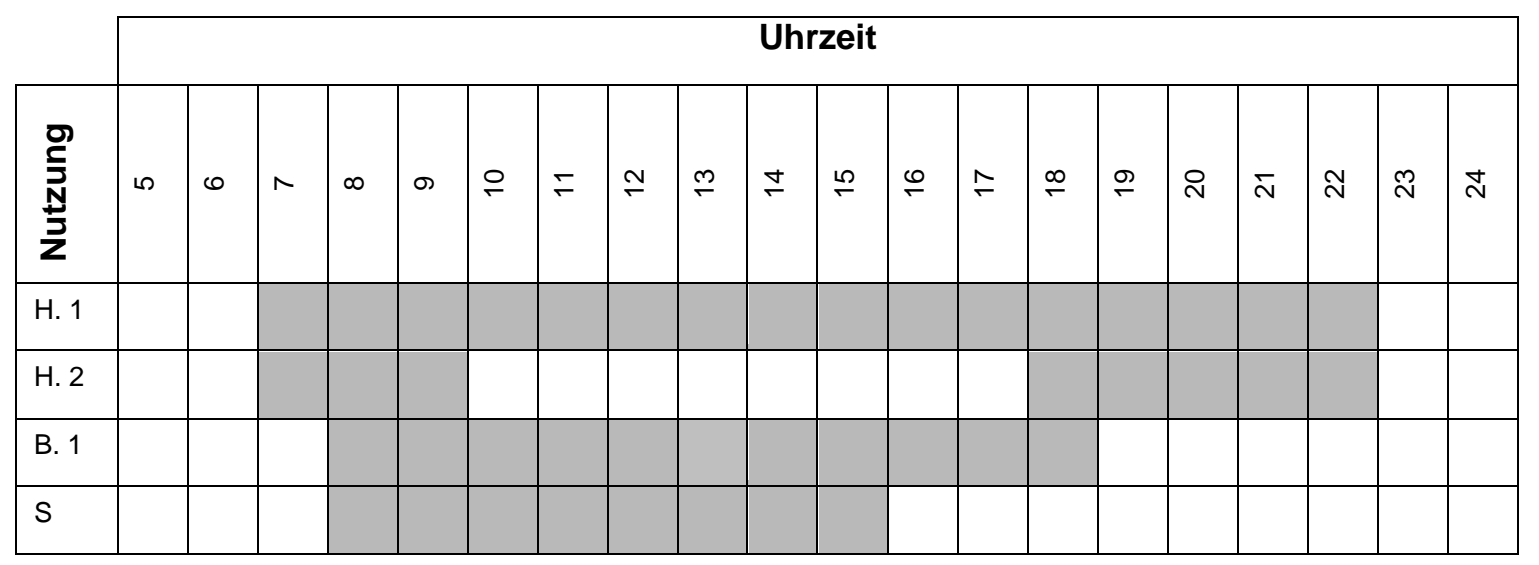

aus der Nutzung resultierende typische Beheizungszeiten aus der Nutzung bedingte Zeiten mit eingeschränktem Heizbetrieb

H. 1 Haushalt mit mehreren Personen und Kindern, Großeltern oder erwerbslosen Personen

H. 2 Haushalt mit erwerbstätigen Personen

B. 1 Büronutzung mit einer Mittagspause von einer Stunde

S Ausbildungsbetrieb mit einer stärkeren Vormittagsnutzung 
Abbildung 5.1 zeigt die Nutzungszeiträume und die damit typischen Beheizungszeiten. Wegen der Trägheit des Beheizungssystems sowie der Trägheit durch die Bauteilspeichermassen wird der Heizbetrieb weit vor der eigentlichen Nutzung gestartet, um rechtzeitig behagliche Verhältnisse zu erzielen.

Zudem sind bei Nichtanwesenheit an Wochenenden und in Urlaubszeiten Beheizungsunterbrechungen normal. Diese führen zu großen Temperaturabsenkungen. Die Wiederanheizphase nach einer solchen Unterbrechung dauert entsprechend lange.

Neben den ständig beziehungsweise zyklisch genutzten Räumen gibt es Räume, welche sehr unregelmäßig genutzt werden, wie beispielsweise Gästezimmer, Ferienwohnungen, Hobbyräume und ähnliche. Bei diesen Räumen ist eine kurze Anheizphase auch bei niedrigen Ausgangstemperaturen erwünscht, da sonst die Nutzung mit zu langen Unbehaglichkeitsphasen einhergeht.

Nach der EnEV $2007 \S 1$ sind folgende Gebäude mit einer geringen Nutzungsdauer von den sonst geforderten energetischen Maßnahmen befreit [37], [38]:

- Provisorische Gebäude mit einer geplanten Nutzungsdauer von bis zu zwei Jahren,

- Gebäude, die dem Gottesdienst oder anderen religiösen Zwecken gewidmet sind, $\left.{ }^{*}\right)$

- Wohngebäude, die für eine Nutzungsdauer von weniger als vier Monaten jährlich bestimmt sind

- sonstige handwerkliche, landwirtschaftliche, gewerbliche und industrielle Betriebsgebäude, die nach ihrer Zweckbestimmung auf eine Innentemperatur von weniger als 12 Grad Celsius oder jährlich weniger als vier Monate beheizt sowie jährlich weniger als zwei Monate gekühlt werden.

*) Die hier gemachte Ausnahmeregelung für Gebäude, welche religiösen Zwecken dienen, kann aus dem Kontext heraus nicht verstanden werden. Wahrscheinlich sind die Begründungen in der nur temporäre Beheizung und den oftmals denkmalgeschützten Gebäuden zu sehen. 


\subsection{Intermittierender Heizbetrieb}

Eine intermittierende Beheizung wird praktisch immer vorgenommen, wenn Gebäude beziehungsweise Gebäudeabschnitte temporär oder zu unterschiedlichen Zwecken (auch Tagund Nachtsituation in Wohnhäusern) genutzt werden. In Zeiten, wo keine Nutzung oder eine eingeschränkte Nutzung mit einem geringeren oder anderem Komfortanspruch (Nachtbetrieb) stattfindet, wird der Heizbetrieb eingeschränkt. Dies geschieht auch mit dem Ziel oder der Hoffnung, Energie einzusparen - je nach Bauweise kann dies mehr oder weniger gelingen oder auch gegenteilig sein.

Der Wärmeverlust eines Gebäudes ist bedingt proportional zur mittleren Temperaturdifferenz des Innen- und Außenklimas. Durch Temperaturabsenkung kann in den Nichtnutzungszeiten zunächst Energie eingespart werden. Die Energieeinsparung ist durch eine kleinere relative Temperaturdifferenz (zu einer Beheizungssituation) zwischen der Raumtemperatur und der Außenlufttemperatur gegeben. Von großer Bedeutung ist hierbei die immer wieder einzubringende Energie für die Wärmespeicher (Wand, Decke und Boden eines intermittierend beheizten Raumes), welche leider nutzlos während der Nachtzeit beziehungsweise Nichtnutzung ohne Heizbetrieb abfließt. Die Raumtemperatur sollte nicht beliebig abgesenkt werden, damit die Baukonstruktion sowie technische Anlagen nicht in kritische Temperaturbereiche gelangen. So steigt mit zunehmender Temperaturabsenkung das Schimmelpilzrisiko an und in Baukonstruktionen können kritische Tauwassermengen auftreten.

\section{Für Wohnräume sind folgende Beheizungsphasen typisch:}

- die morgendliche Aufheizzeit

- Beheizung in der Tag-Nutzungszeit

- die Abkühlzeit ohne oder mit geringer Nachbeheizung

Nach DIN V 4108-6 können die Energieeinsparungen für einen unterbrochenen Heizbetrieb ermittelt werden. Untersuchungen von Hauser [6], welche auf dem Monatsbilanzverfahren der DIN 4108-6 und Simulationen beruhen, zeigen, dass bei einer Nachtabsenkung Energie eingespart werden kann. In diesen Untersuchungen sind leichte mit schweren Gebäudekonstruktionen verglichen worden. Hierbei wurde deutlich, dass durch Nachtabsenkungen eine größere Energieeinsparung bei leichten Baukonstruktionen gegenüber schweren Baukonstruktionen zu erwarten ist. 


\subsection{Die Aufheizphase}

Durch eine intermittierende Beheizung kommt es zu entsprechenden Temperaturabsenkungen, so dass bei einer normalen Nutzung ein Aufheizen des Raumes immer wieder erfolgen muss. Es muss praktisch die gewünschte operative Temperatur im Raum erzielt werden, so dass die Raumlufttemperatur und die Raumoberflächentemperatur angehoben werden müssen. und zudem ein zusätzlicher Wärmeabfluss nach außen durch Transmission und Lüftung auftritt, welcher durch die Beheizung kompensiert werden muss. Eine Gegenüberstellung der „Wärmeabflüsse“ nach außen und der für die „Wiederauffüllung der Wärmespeicher“ erforderlichen Energiemengen zeigt, dass für die „Wiederauffüllung der Wärmespeicher“ zumindest bei einem guten Dämmniveau ein sehr großer Energieaufwand erforderlich ist, vergleiche Kapitel 8.5.

Zudem benötigt dieser Aufheizvorgang Zeit und diese ist von den Gebäudekonstruktionen, der Heizleistung und den Wärmeübergabesystemen (Heizkörper, Fußbodenheizung oder andere) abhängig.

In der neuen DIN EN ISO 12831:2003 [36], welche die DIN 4701:1983 [39] ersetzt, wird die zusätzlich benötigte Heizleistung vereinfacht berechnet mit:

$\Phi_{R H, i}=A_{i} \cdot f_{R H}$

Formel 5.1: Zusätzlich benötigte Aufheizleistung

mit:

$\begin{array}{lll}\phi_{R H, i} & {[W]} & \text { zusätzlich benötigte Aufheizleistung } \\ A_{i} & {\left[m^{2}\right]} & \text { Bodenfläche des betrachteten Raumes } \\ f_{R H} & {[-]} & \text { Korrekturfaktor abhängig von der erwünschten Aufheizzeit, } \\ & & \text { der Temperaturabsenkung und der Bauweise }\end{array}$

Der Faktor $\mathrm{f}_{\mathrm{RH}}$ wird in DIN EN 12831 [36] tabellarisch für Wohn- und Nichtwohngebäude angegeben. Neben dem Temperaturabfall über die Heizungsunterbrechung bestimmt die erwünschte Wiederaufheizzeit in Stunden den Faktor $\mathrm{f}_{\mathrm{RH}}$.

Die Einteilung der Gebäudemasse wird nach DIN EN 12831 [36] pauschal wie folgt vorgenommen:

- schwere Gebäude

- leichte Gebäude 
Die Norm-Heizlast für einen Raum ist wie folgt zu bestimmen:

$\Phi_{H L, i}=\Phi_{T, i}+\Phi_{V, i}+\Phi_{R H, i}$

Formel 5.2: Norm-Heizlast für einen Raum

mit:
$\phi_{T, \mathrm{i}} \quad[\mathrm{W}] \quad$ stationärer Transmissionswärmeverlust des betrachteten Raumes
$\phi \mathrm{V}, \mathrm{i} \quad[\mathrm{W}] \quad$ Lüftungswärmeverlust für den betrachteten Raum
$\phi_{\mathrm{RH}, \mathrm{i}} \quad[\mathrm{W}] \quad$ zusätzlich Aufheizleistung für den betrachteten Raum

Bei einer intermittierenden oder seltenen Beheizung ist oftmals eine schnelle Wiederaufheizung des Raumes erwünscht. Die benötigte Zeit der Wiederaufheizung hängt von folgenden Randbedingung ab:

- Raumgröße

- Heizleistung

- Wärmeübertragung

- Speichermasse der Bauteile

- Wärmeverluste während der Anheizzeit

- Luftwechsel

Für die Wiederaufheizung eines Raumes sind insbesondere die oberflächennahen Bauteilschichten von großer Bedeutung. Diese beeinflussen in starkem Maße die benötigte Wiederaufheizzeit und -energiemenge.

Die thermischen Materialeigenschaften, welche für die Wärmeaufnahme entscheidend sind, können bei homogenen Verhältnissen durch den Wärmeeindringkoeffizient $b$ dargestellt werden.

$b=\sqrt{\lambda \cdot c \cdot \rho}$

Formel 5.3: Wärmeeindringkoeffizient $b$

mit:

$\begin{array}{lll}\text { b } & {\left[\frac{\mathrm{W}}{\mathrm{m}^{2} \cdot \mathrm{s}^{0,5}}\right]} & \text { Wärmeeindringkoeffizient } \\ \lambda & {\left[\frac{\mathrm{W}}{\mathrm{m} \cdot \mathrm{K}}\right]} & \text { Wärmeleitfähigkeit der oberflächennahen Schicht } \\ \text { C } & {\left[\frac{\mathrm{J}}{\mathrm{kg} \cdot \mathrm{K}}\right]} & \text { spezifische Wärmekapazität der oberflächennahen Schicht } \\ \rho & {\left[\frac{\mathrm{kg}}{\mathrm{m}^{3}}\right]} & \text { Rohdichte der oberflächennahen Schicht }\end{array}$


Je kleiner der Wärmeeindringkoeffizient $b$ eines Stoffes ist, umso schneller erfolgt die Erwärmung seiner Oberfläche.

Für einen homogenen Baustoff kann der Anstieg der Lufttemperatur $\theta_{\mathrm{Li}}$ und der Oberflächentemperatur $\theta_{\mathrm{O}}$ nach Krischer und Kast [40] mit folgender Gleichung in Abhängigkeit von der Zeit $t$ angegeben werden.

$\theta_{L i(t)}=\theta_{O i(0)}+q_{0}\left(\frac{1}{\alpha i}+\frac{2}{\sqrt{\pi}} \cdot \frac{1}{b} \cdot \sqrt{t}\right)$

Formel 5.4: Berechnung der zeitabhängigen Lufttemperatur nach Krischer und Kast

und

$\theta_{O i(t)}=\theta_{O i(0)}+q_{0} \cdot \frac{2}{\sqrt{\pi}} \cdot \frac{1}{b} \cdot \sqrt{t}$

Formel 5.5: Berechnung der zeitabhängigen Oberflächentemperatur nach Krischer und Kast

mit:

$\begin{array}{lll}\text { b } & {\left[\frac{\mathrm{W}}{\mathrm{m}^{2} \cdot \mathrm{s}^{0,5}}\right]} & \text { Wärmeeindringkoeffizient } \\ \lambda & {\left[\frac{\mathrm{W}}{\mathrm{m} \cdot \mathrm{K}}\right]} & \text { Wärmeleitfähigkeit der oberflächennahen Schicht } \\ \mathrm{C} & {\left[\frac{\mathrm{J}}{\mathrm{kg} \cdot \mathrm{K}}\right]} & \text { spezifische Wärmekapazität der oberflächennahen Schicht } \\ \rho & {\left[\frac{\mathrm{kg}}{\mathrm{m}^{3}}\right]} & \text { Rohdichte der oberflächennahen Schicht } \\ \mathrm{t} & {[\mathrm{h}]} & \text { Zeit }\end{array}$

Mit den angegebenen Formeln kann die Temperatur nach einer definierten Anheizdauer für die Luft- und Oberflächentemperatur vereinfacht ermittelt werden.

Für eine genauere Betrachtung von Wandkonstruktionen mit mehreren Schichten ist die Berechnung über numerische Verfahren oder grafische Verfahren möglich, vergleiche Kapitel 2.4. Der Aufwand für diese Berechnungen ist sehr hoch und wird derzeit durch die Energieeinsparverordnung nicht gefordert. Aus diesem Grund werden genauere Berechnungen des thermischen Verhaltens bei instationären Randbedingungen in der Praxis bisher nur selten vorgenommen. 


\section{Messtechnische Untersuchung der thermischen Entwicklung in Räumen während einer Heizperiode}

\subsection{Vorgehensweise und Randbedingungen}

Innerhalb einer Heizperiode wurden vom 16.11.2009 bis zum 31.03.2010 die Raumlufttemperaturen in 13 Wohn- beziehungsweise Büroräumen mit Datenloggern aufgezeichnet. In Tabelle 6.1 sind neben dem Baujahr, welches eine gewisse Aussage über das energetischen Wärmedämmniveau macht, die Benutzeranzahl und die Art der Bauweise (leicht oder schwer) angegeben.

Im Raum R12 mussten die Messungen aus Datenschutzgründen leider nach einigen Wochen abgebrochen werden.

Die Datenlogger wurden so positioniert, dass kein direkter Strahlungsaustausch mit Heizkörpern und Fenstern gegeben war und sich die Datenlogger auch nicht in unmittelbarer Nähe von Fenstern oder Türen befanden. Pro Objekt kam ein Datenlogger zum Einsatz.

Für das Auskühlverhalten sind die Verglasung einschließlich der temporären Wärmeschutzvorrichtung beim Fenster (Rollladen oder Vergleichbares) wichtig. Neben diesen Faktoren ist die Luftdichtigkeit für die Auskühlung von großer Bedeutung. Durch einen erhöhten Luftwechsel findet eine deutlich schnellere Auskühlung des Raumes statt - auch bei einem hohen Wärmedämmniveau. Diese Situation ist häufig im unsanierten älteren Gebäudebestand anzutreffen.

Die Aufzeichnungen der Raumlufttemperaturen zeigen das Nutzerverhalten, die Dauer der Anheizphasen und den Temperaturabfall bei den Heizungsunterbrechungen. Parallel zur Raumlufttemperatur ist die Außenlufttemperatur an zwei Standorten in Aachen aufgezeichnet worden. Die beiden Außenlufttemperaturen sind gemittelt in den jeweiligen Raumlufttemperaturdiagrammen dargestellt, vergleiche Abbildungen 6.1 bis 6.13 .

Beachtlich sind die großen Raumlufttemperaturschwankungen, welche in den meisten der untersuchten Räume während der beobachteten Heizperiode festzustellen sind, vergleiche Tabelle 6.1. Bei allen aufgezeichneten Raumlufttemperaturen sind die Nachtabsenkungen deutlich erkennbar. Bei den Büroräumen ist neben der Nachtabsenkung auch die Wochenendabsenkung zu erkennen.

Neben den zyklischen Absenkungen sind auch Absenkungen durch längere Abwesenheiten der Benutzer erkennbar, beispielsweise aufgrund von längeren Urlaubszeiten. 


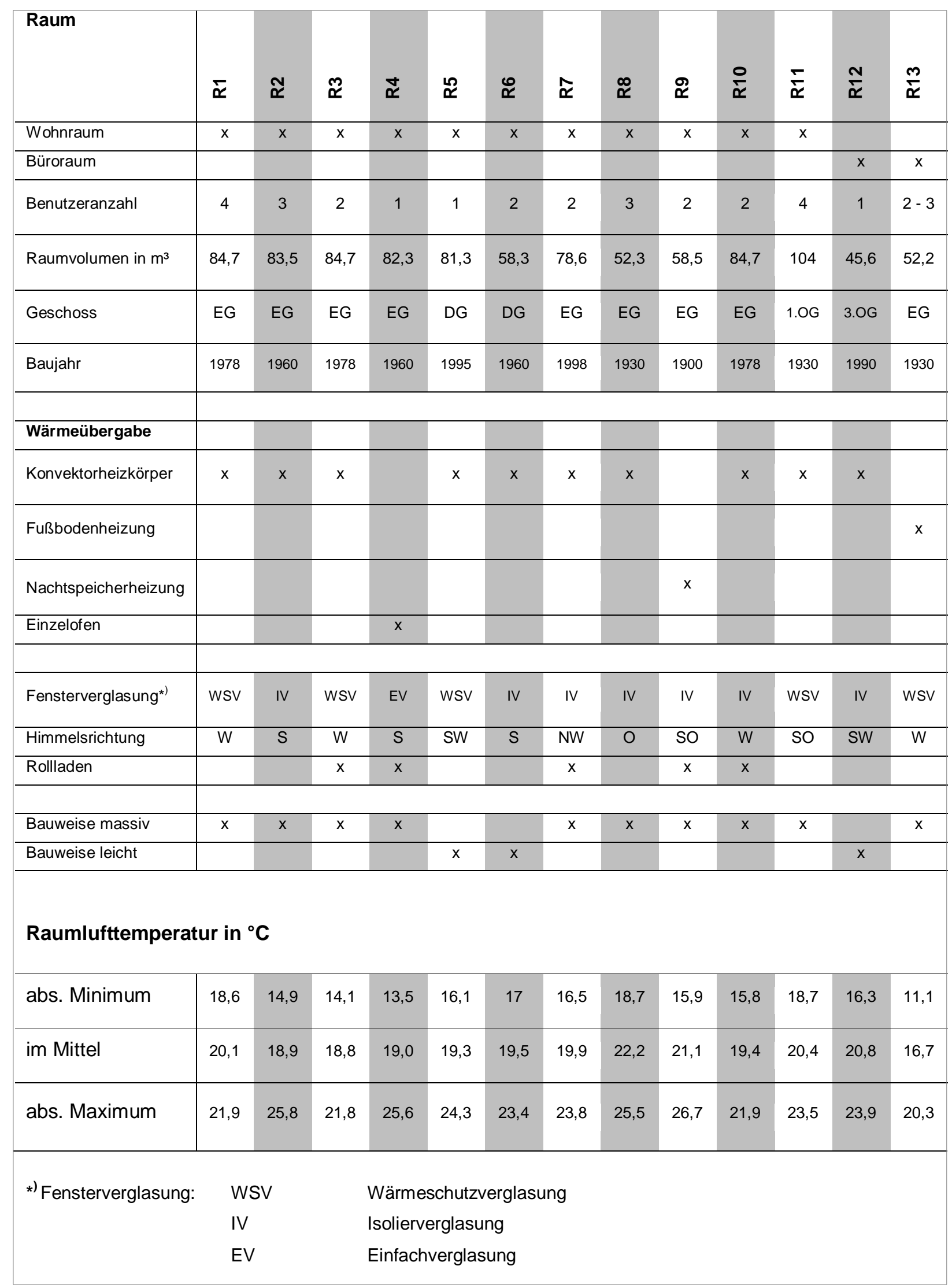

Tabelle 6.1: Angaben zu den 13 untersuchten Räumen 


\subsection{Raumbeheizung in Wohngebäuden}

Es sind insgesamt 11 Wohnräume untersucht worden. Die Abbildungen 6.1 bis 6.11 zeigen die Raumtemperaturentwicklung über den Beheizungszeitraum. Der Bereich „A“ kennzeichnet einen typischen vergrößerten Ausschnitt aus dem Temperaturverlauf.

Die Wohnräume R4 und R9 weisen große Temperaturschwankungen auf. Diese Räume sind dezentral beheizt. Eine gute Temperaturregelung ist beim Einzelofen sowie beim Nachtspeicherofen nicht möglich, so dass Über- und Untertemperierungen auftreten. 


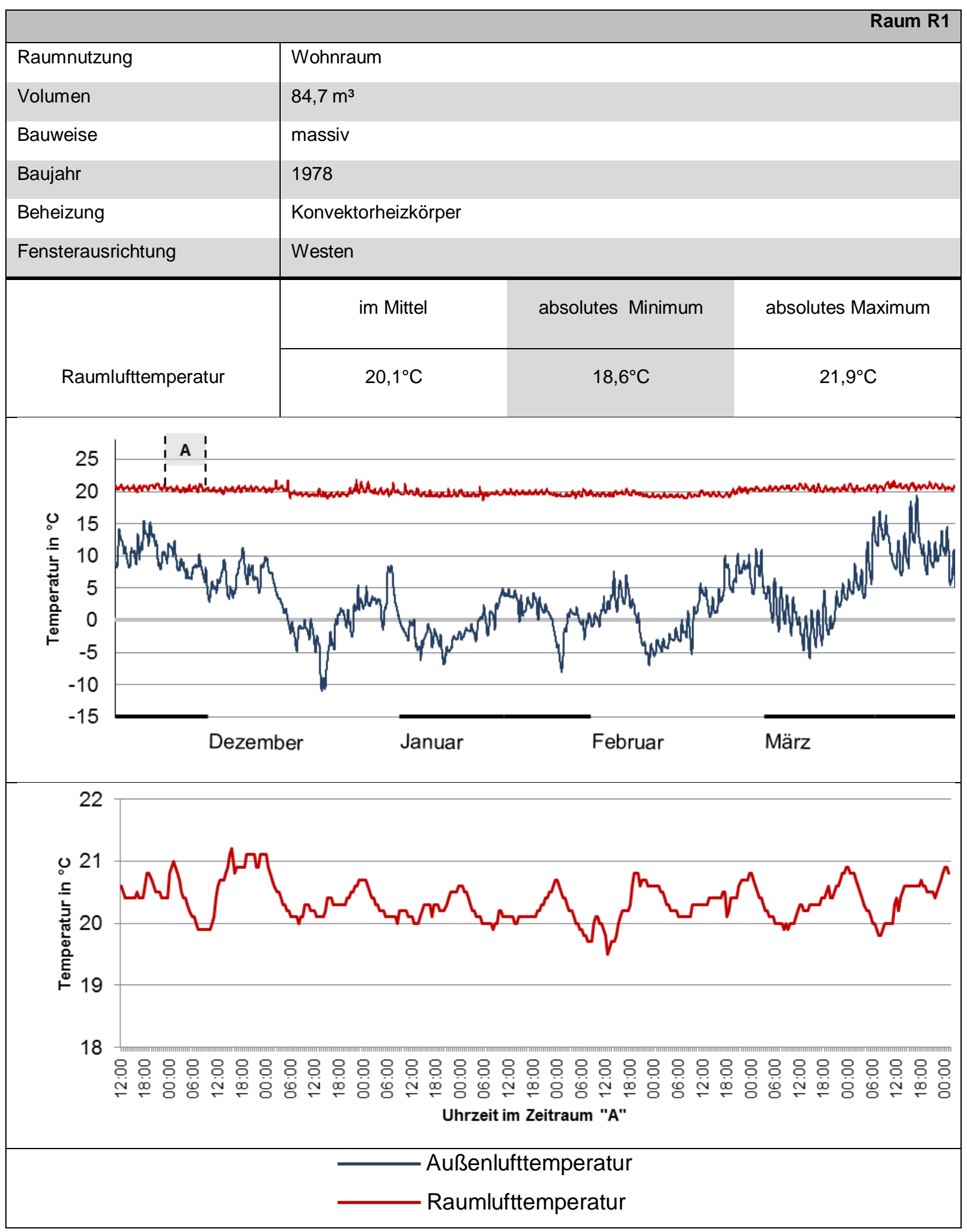

Abbildung 6.1: Raum- und Außenlufttemperatur für den Raum R1 


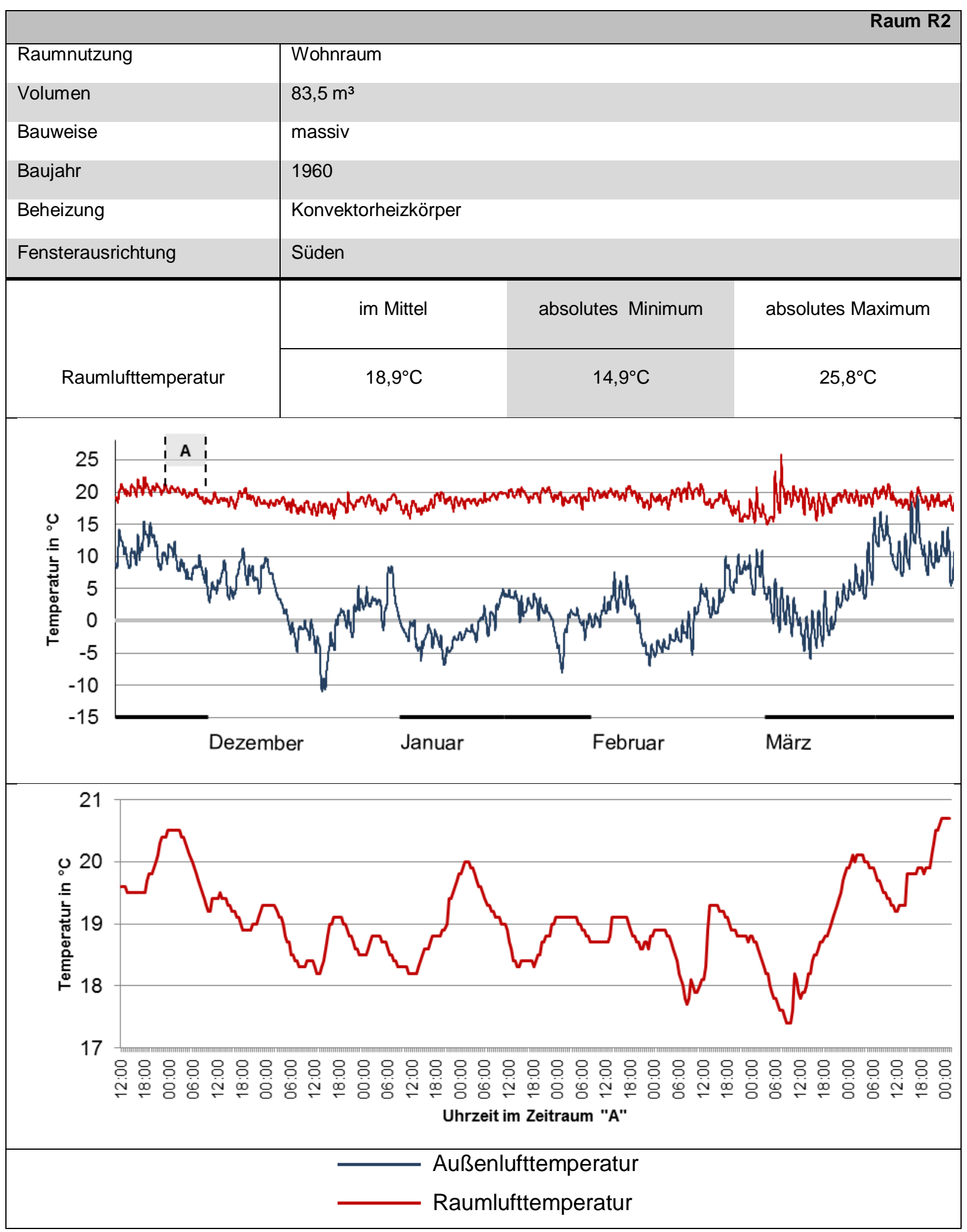

Abbildung 6.2: Außen- und Raumlufttemperatur für den Raum R2 


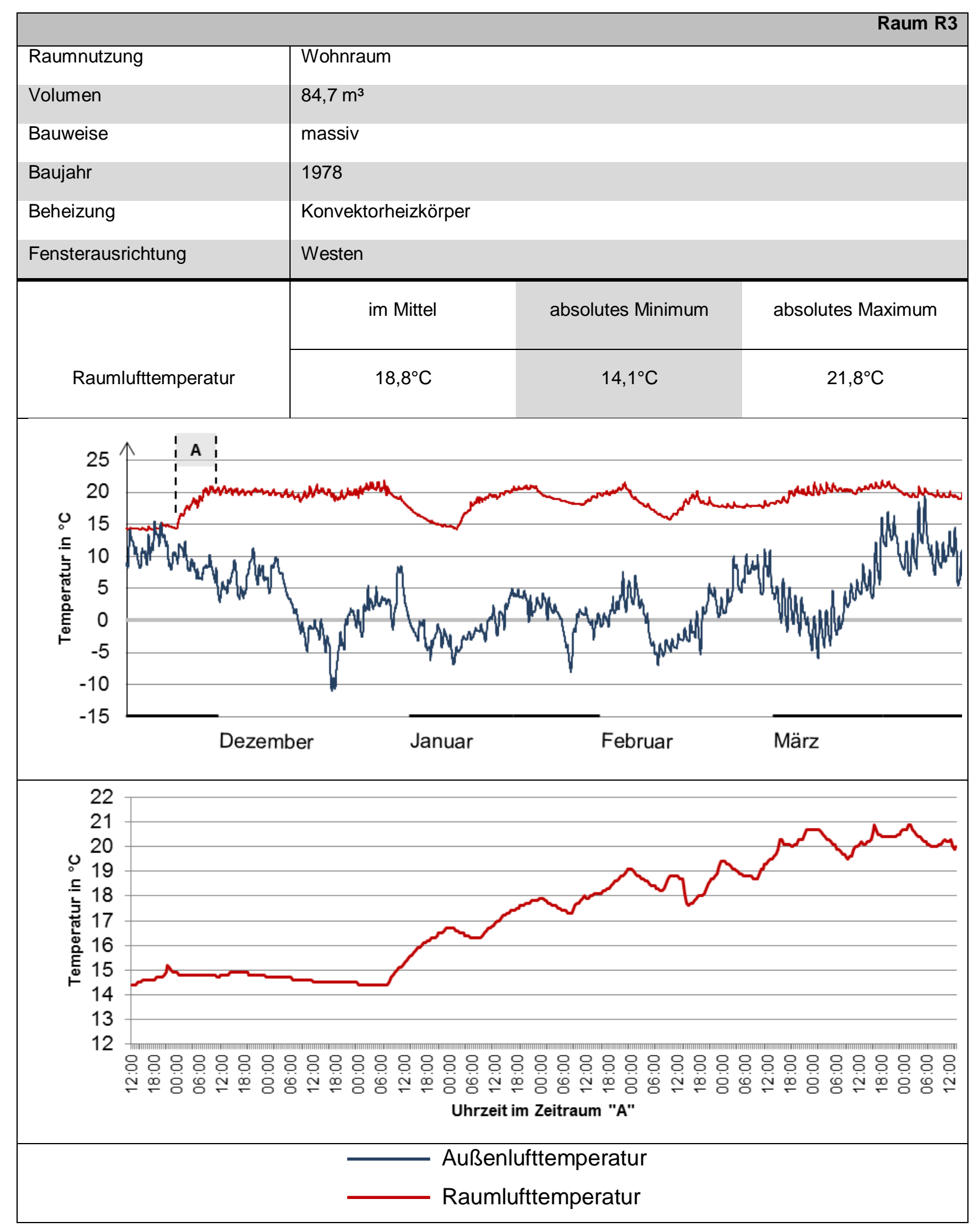

Abbildung 6.3: Außen- und Raumlufttemperatur für den Raum R3 


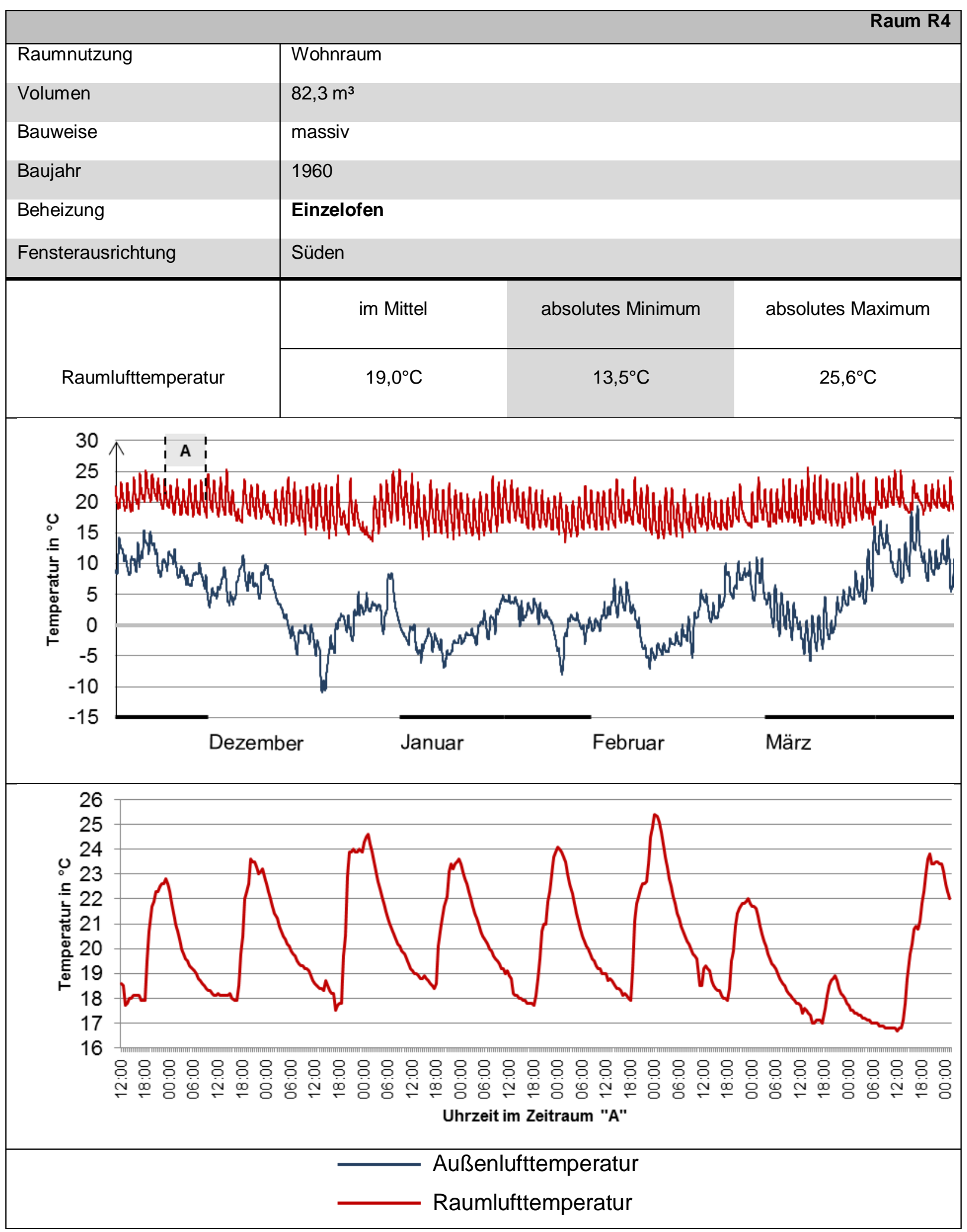

Abbildung 6.4: Außen- und Raumlufttemperatur für den Raum R4 


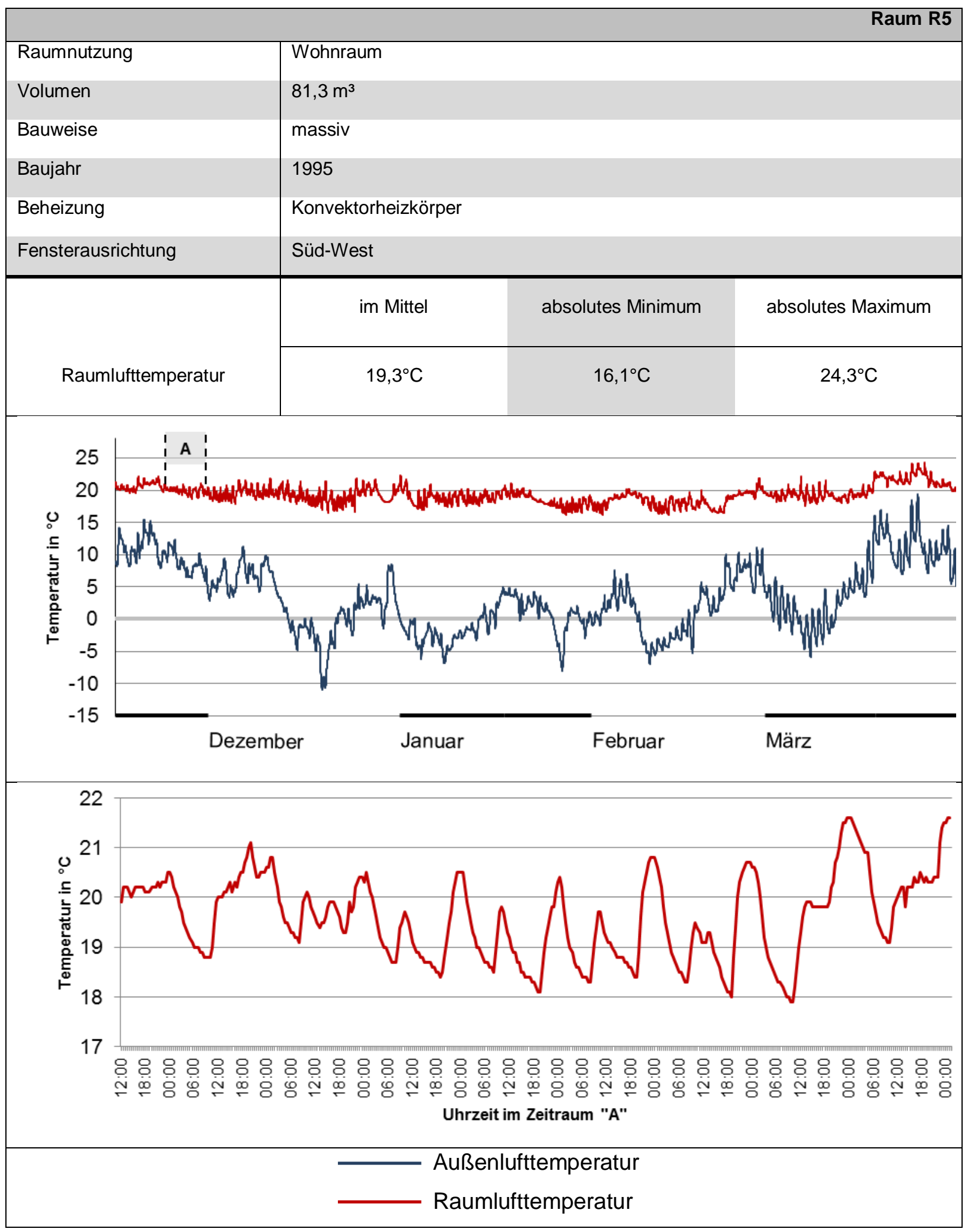

Abbildung 6.5: Außen- und Raumlufttemperatur für den Raum R5 


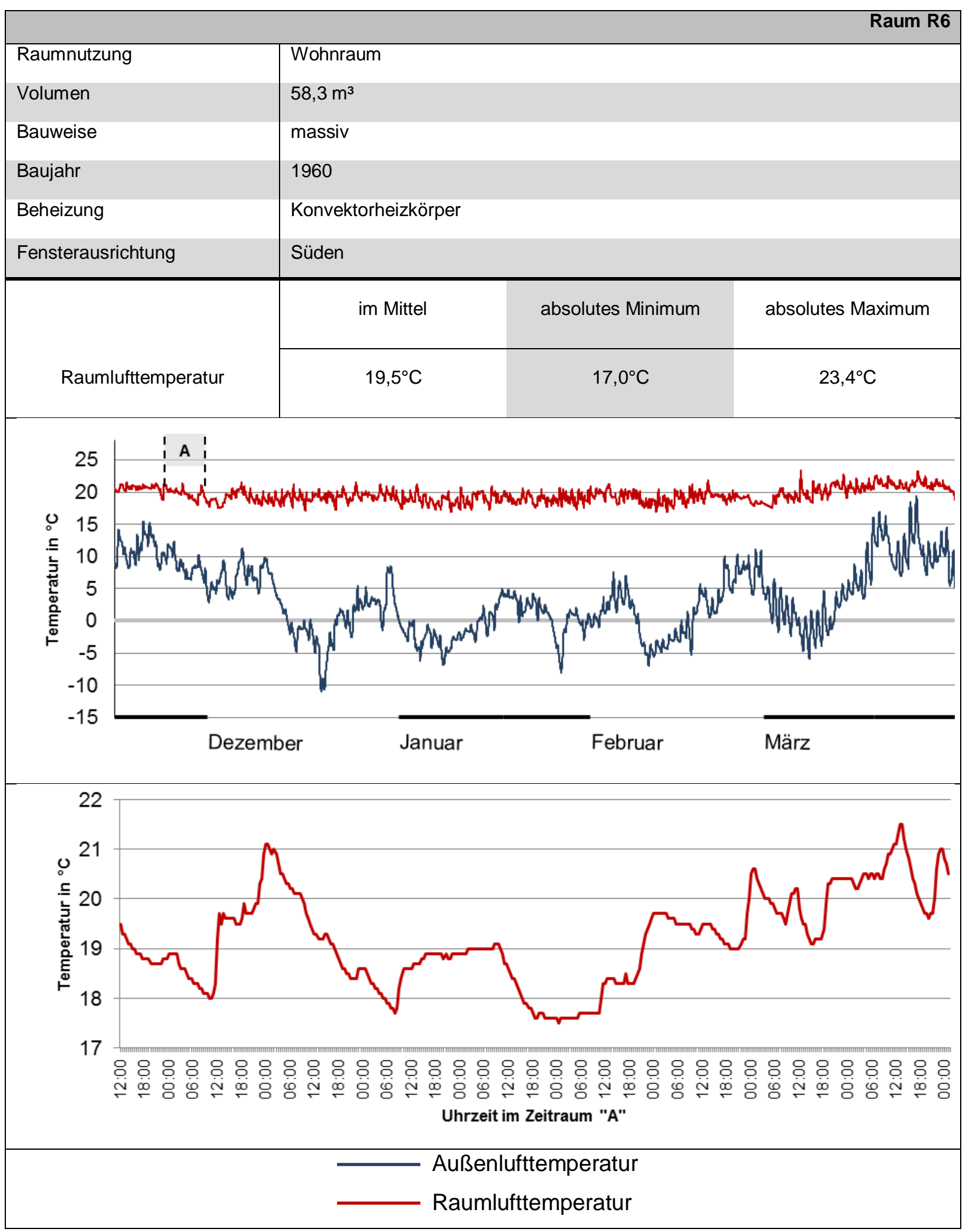

Abbildung 6.6: Außen- und Raumlufttemperatur für den Raum R6 


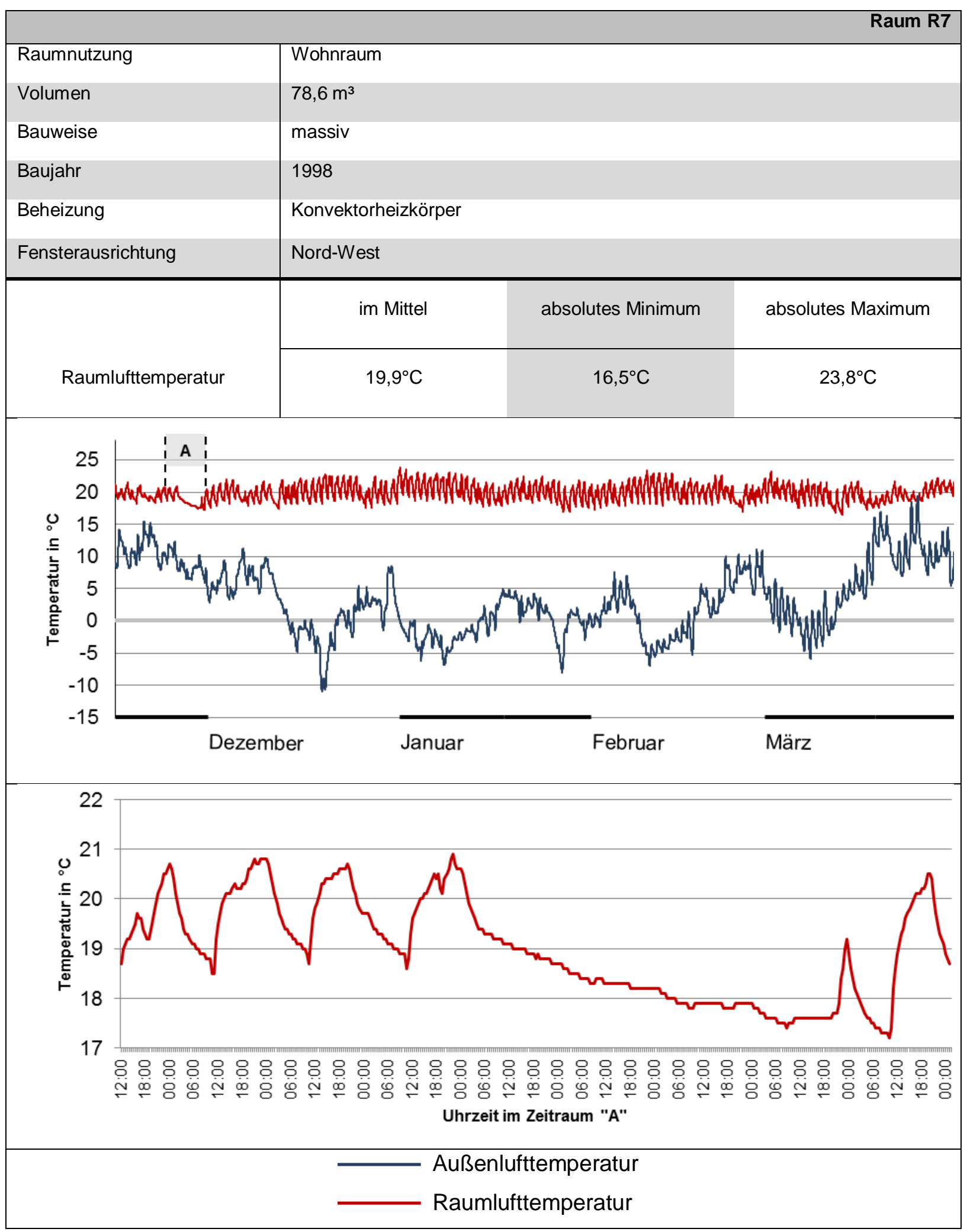

Abbildung 6.7: Außen- und Raumlufttemperatur für den Raum R7 


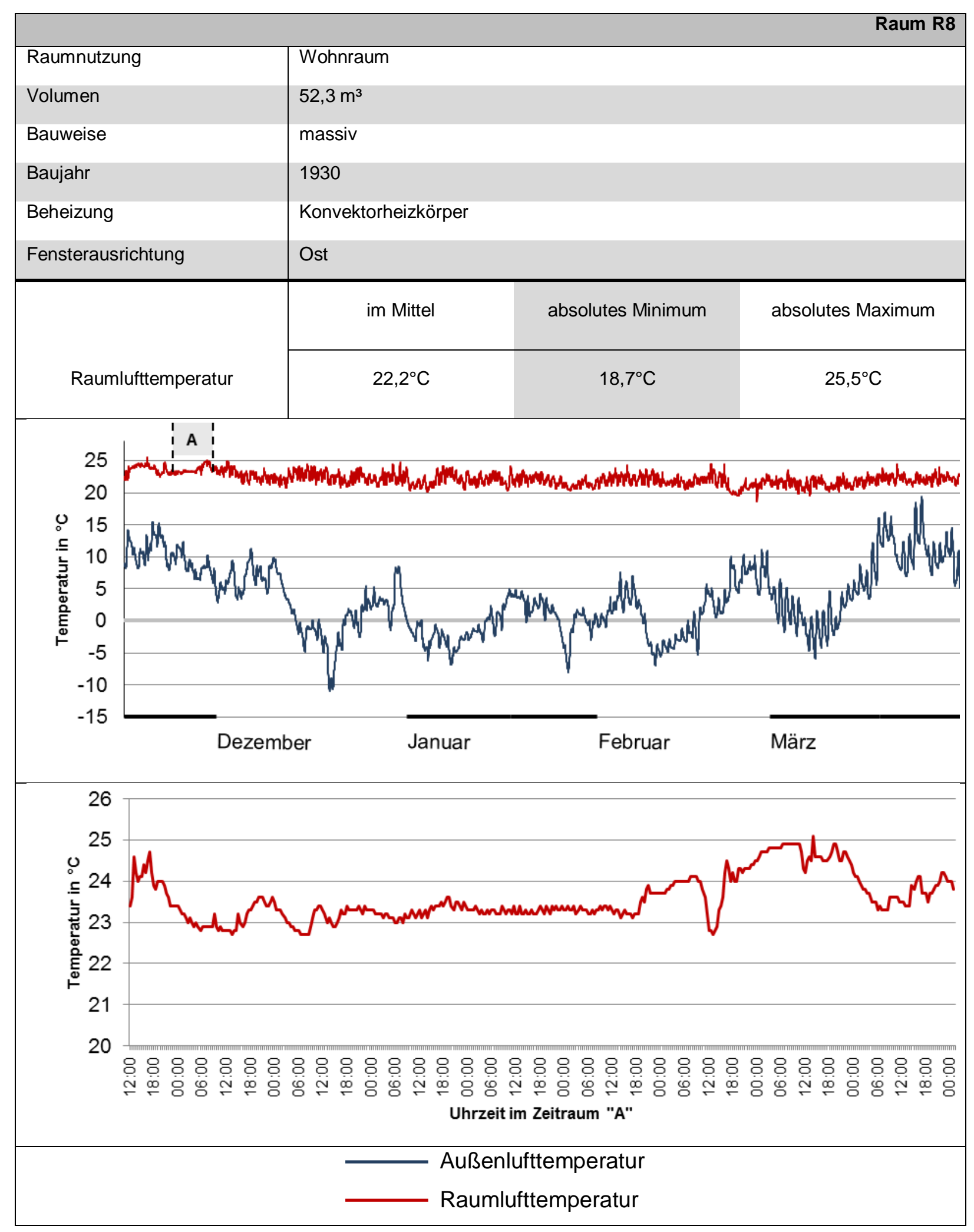

Abbildung 6.8: Außen- und Raumlufttemperatur für den Raum R8 


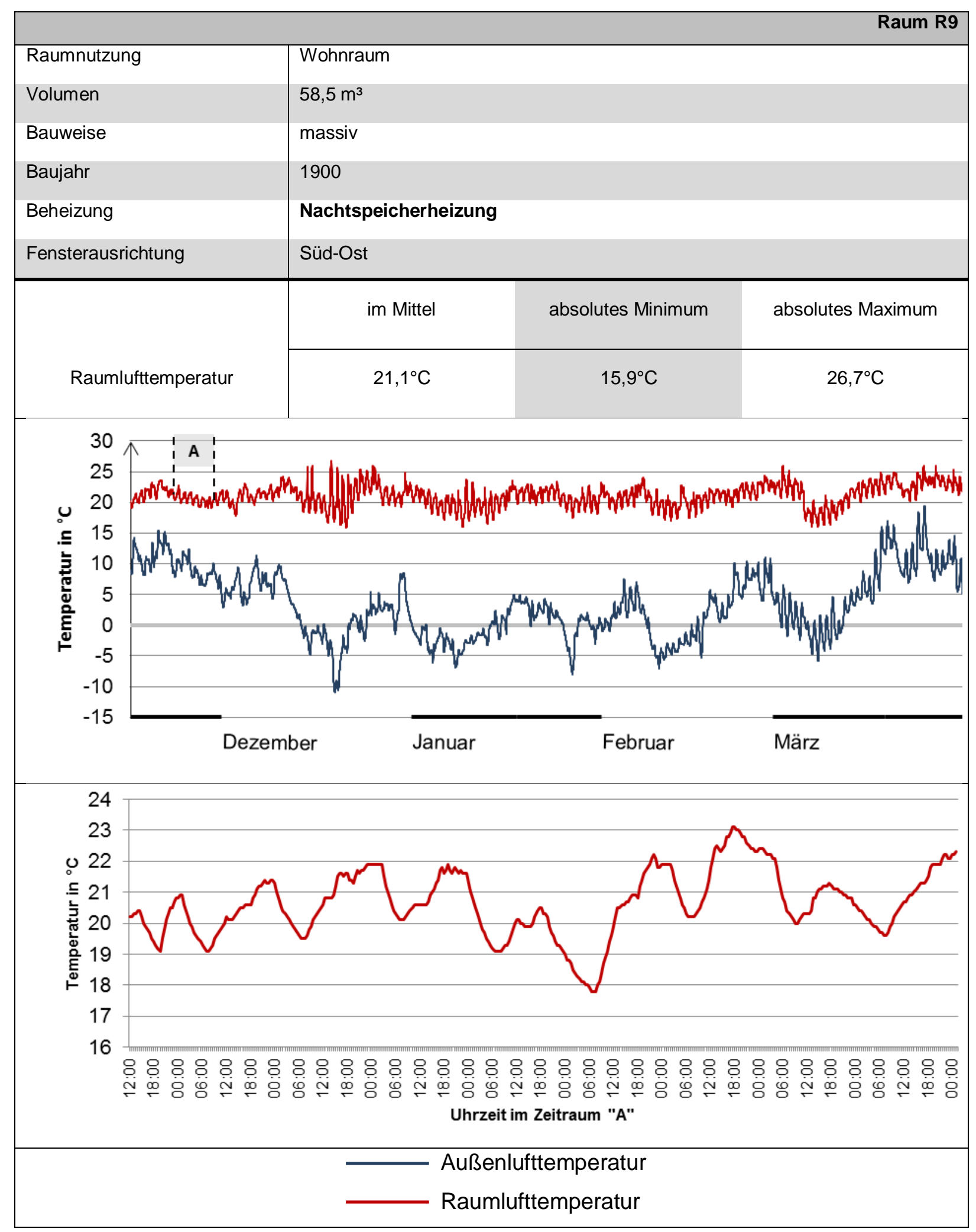

Abbildung 6.9: Außen- und Raumlufttemperatur für den Raum R9 


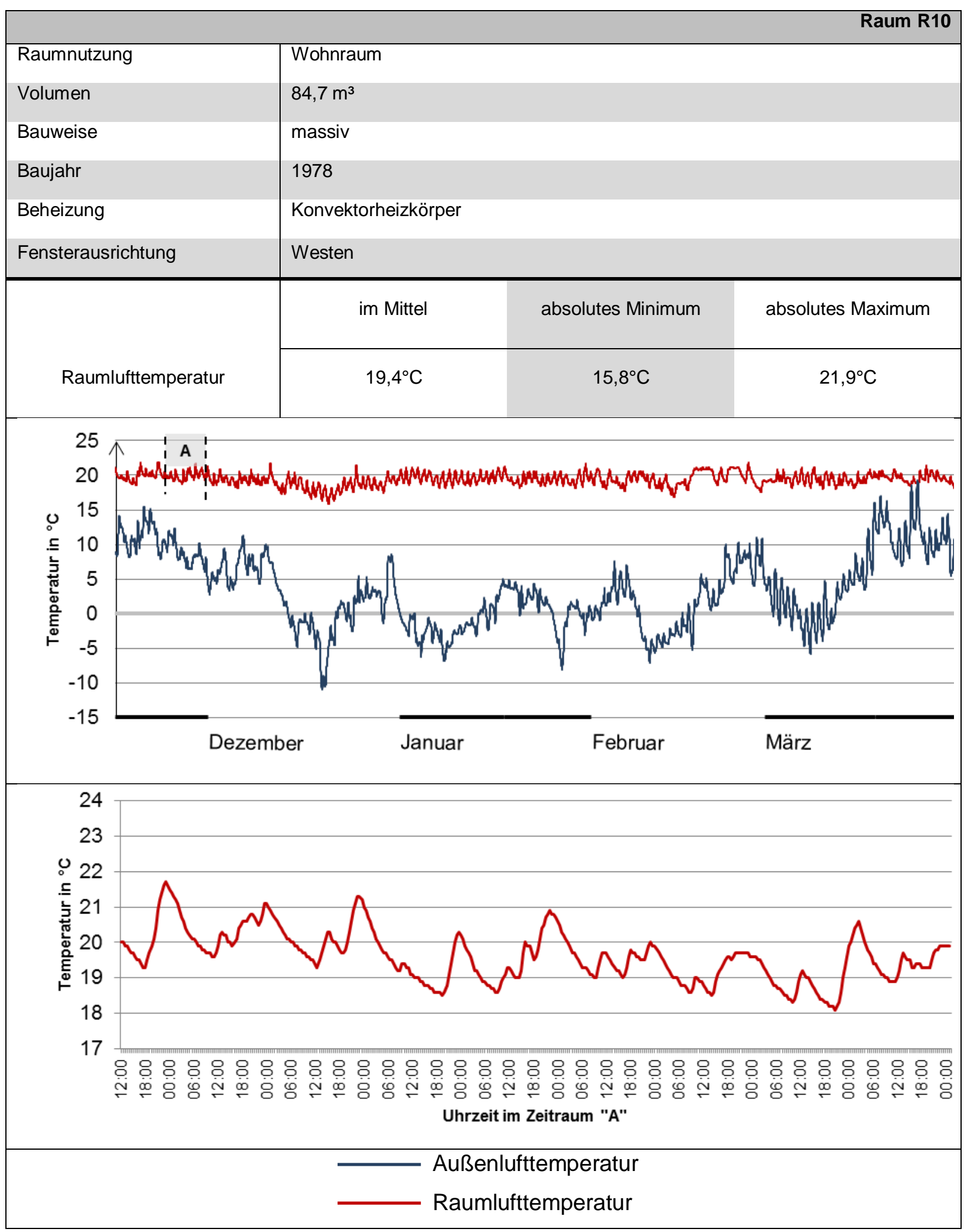

Abbildung 6.10: Außen- und Raumlufttemperatur für den Raum R10 


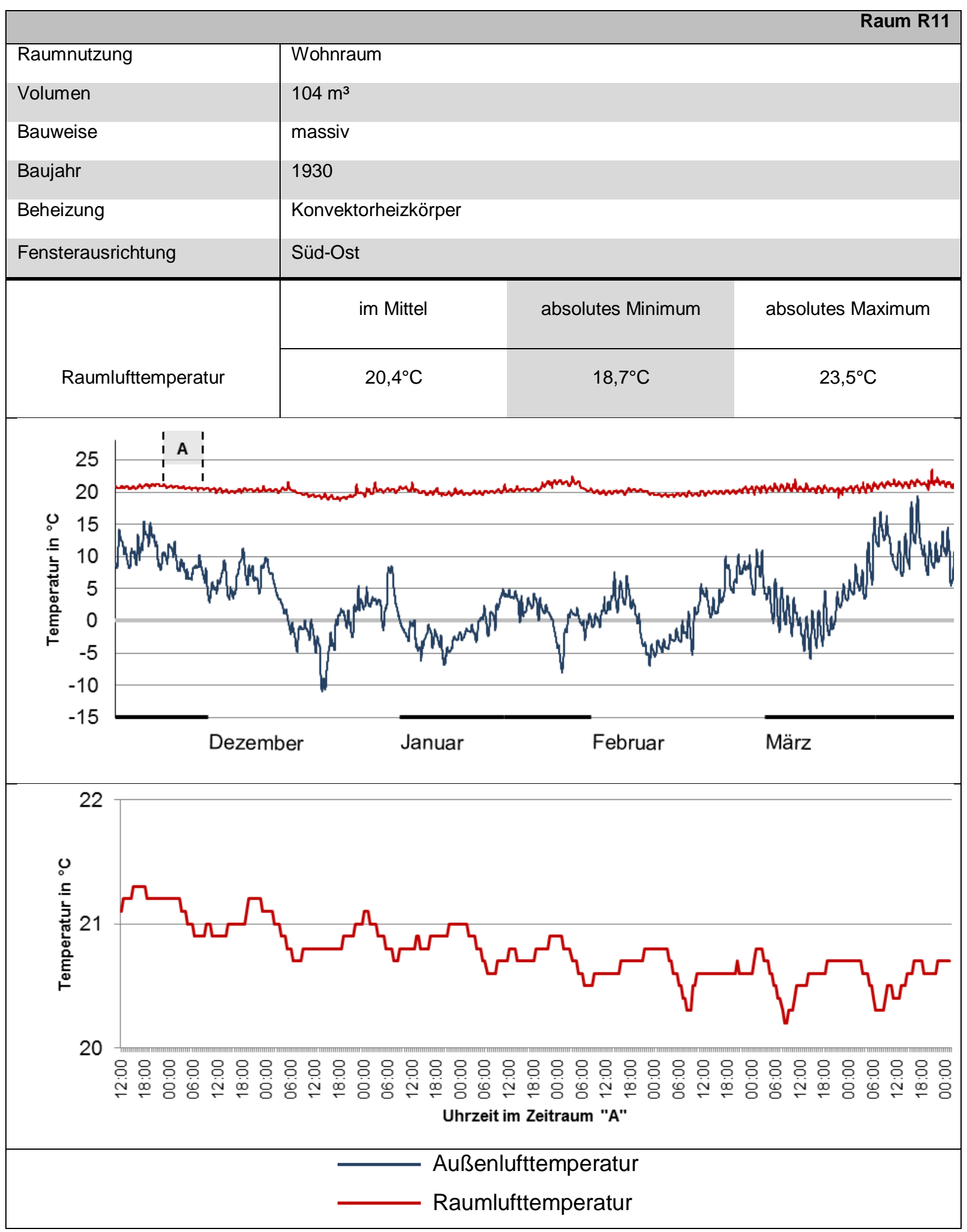

Abbildung 6.11: Außen- und Raumlufttemperatur für den Raum R11 


\subsection{Raumbeheizung in Bürogebäuden}

Die beiden untersuchten Büroräume R12 und R13 zeigen einen Wochenzyklus in der Beheizung. Hier sind die Arbeitszeit und die Nichtnutzungzeiten der Räume zur Nachtzeit sowie am Wochenende erkennbar, vergleiche Abbildung 6.12 - 6.14.

Betrachtet man die vergrößerten Zeitabschnitte in den Abbildungen 6.12 und 6.13, so wird deutlich, dass die Anheizung zu Wochenbeginn wegen des größeren Temperaturabfalls am Wochenende besonders lange dauert. Es zeigt sich, dass die Temperaturen, welche an den anderen Wochentagen vorhanden sind, am ersten Tag nach einem Wochenende nicht erreicht werden. 


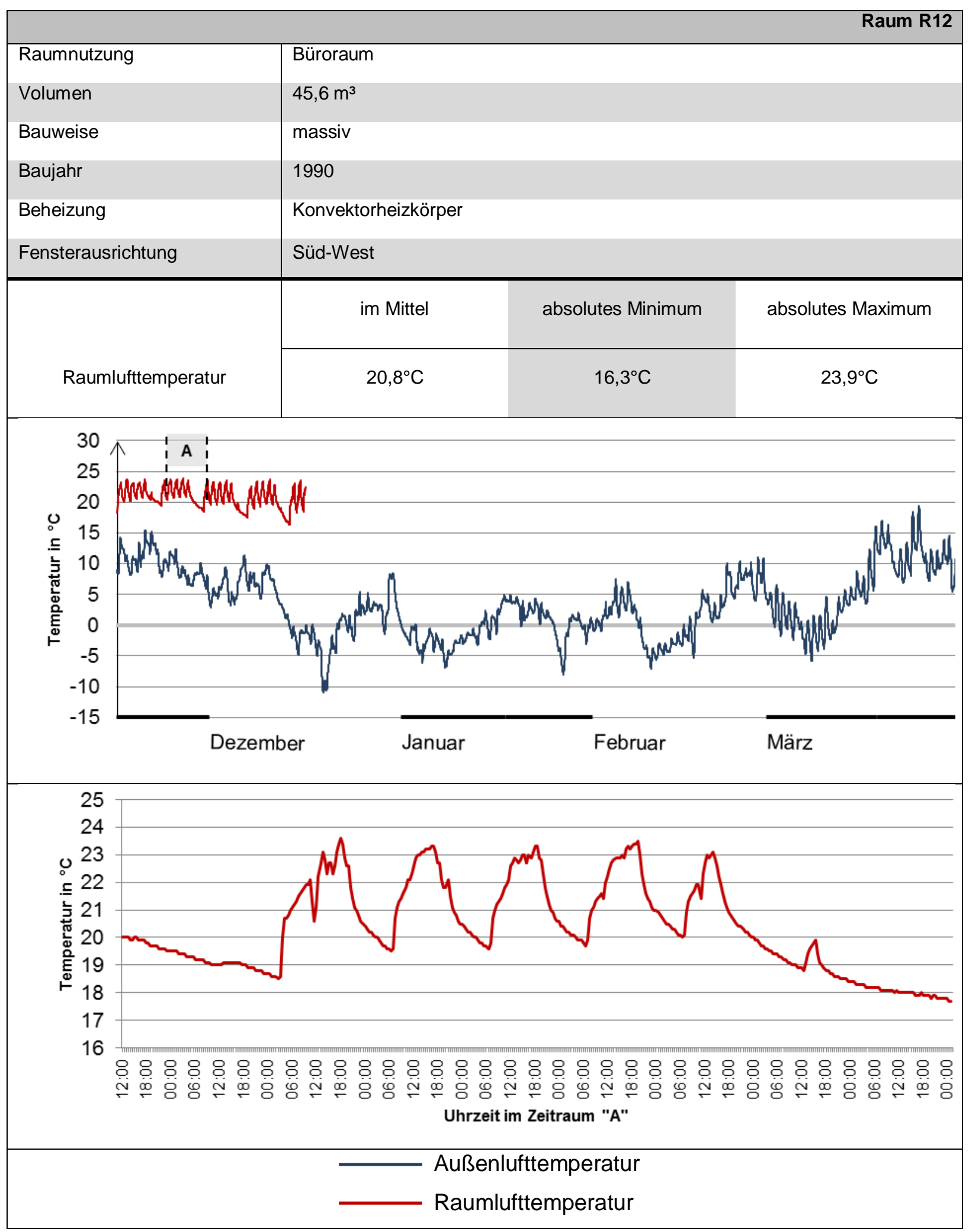

Abbildung 6.12: Außen- und Raumlufttemperatur für den Raum R12 


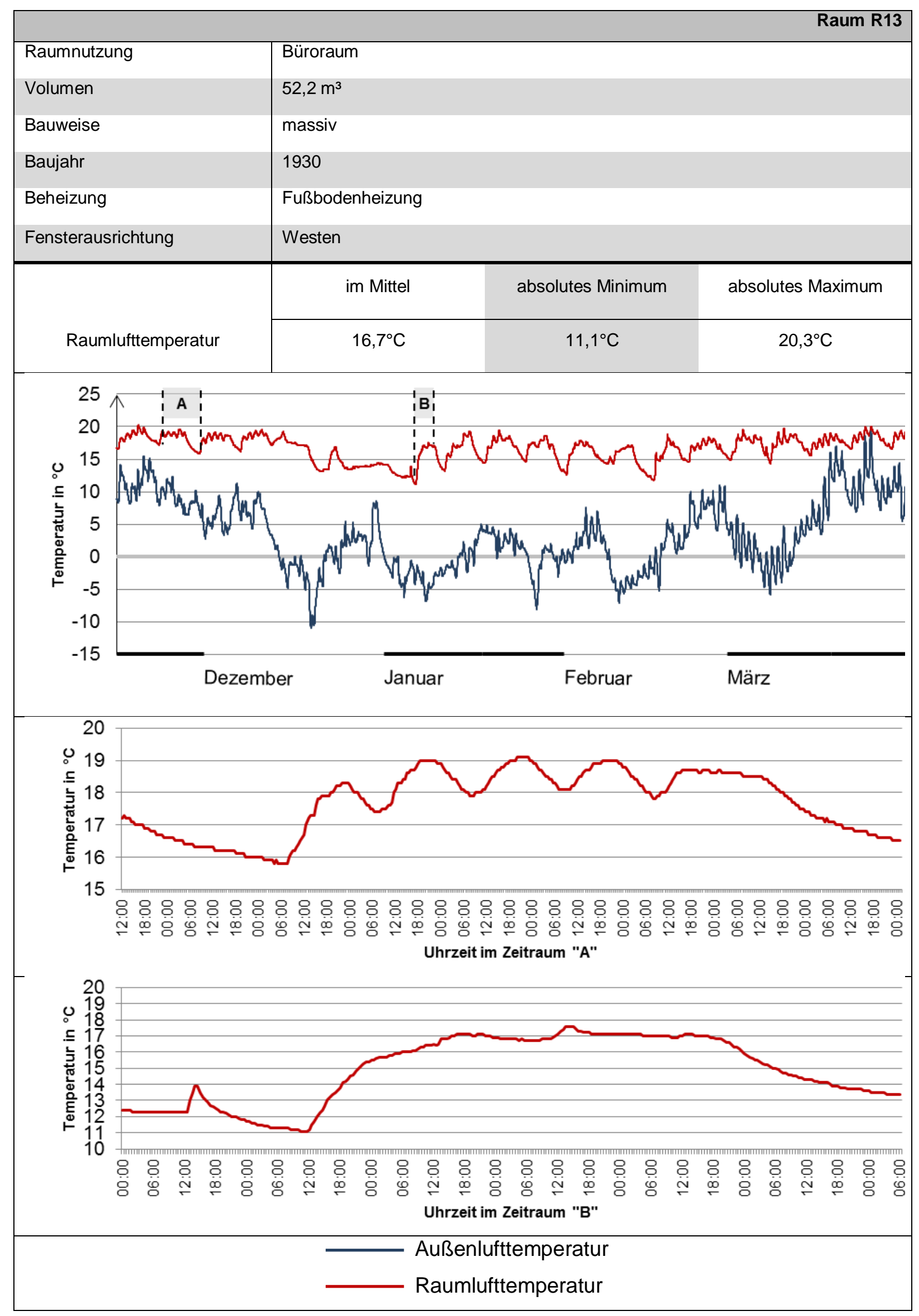

Abbildung 6.13: Außen- und Raumlufttemperatur für den Raum R13 


\subsection{Zusammenfassung der vorgefundenen Verhältnisse}

Bei den Untersuchungen zeigen sich keine direkt erkennbaren Zusammenhänge zwischen den Raumlufttemperaturen und Außenlufttemperaturen. Die aufgezeichneten Raumlufttemperaturen belegen, dass in den untersuchten Fällen kein stationärer Heizbetrieb, mit Ausnahme der Situation im Raum R1, stattgefunden hat.

Betrachtet man die in Tabelle 6.2 zusammengefassten Raumlufttemperaturen, so wird ersichtlich, dass eine Behaglichkeit im Sinne der Anforderungen nach ISO 7730 [33] beziehungsweise den Norm-Innenraumtemperaturen nach DIN EN 12831 [36] zum Großteil der Nutzungszeit nicht erreicht wurde.

Weiterhin zeigen die Aufzeichnungen, dass oftmals eine Nichtbeheizung der Räume gegeben ist. In einigen der untersuchten Objekte sind die Nichtnutzungszeiten auch gleich beziehungsweise größer als die eigentlichen Beheizungszeiten.

Stellt man den Zeitanteil der Raumlufttemperaturen anteilsmäßig dar, sind in den untersuchten Räumen, welche auch bewusst verschiedene Nutzungsverhältnisse erfassen sollten, sehr unterschiedliche thermische Gegebenheiten zu erkennen, vergleiche Tabelle 6.2.

\begin{tabular}{|c|c|c|c|c|c|c|}
\hline \multirow[t]{2}{*}{ Raum } & \multicolumn{3}{|c|}{ Zeitanteil der Raumlufttemperaturen } & \multicolumn{3}{|c|}{ Raumlufttemperatur in ${ }^{\circ} \mathbf{C}$} \\
\hline & $<19^{\circ} \mathrm{C}$ & $>19^{\circ} \mathrm{C}$ & $>20^{\circ} \mathrm{C}$ & Im Mittel & Minimum & Maximum \\
\hline $\mathrm{R} 1$ & $0,6 \%$ & $99,4 \%$ & $50,9 \%$ & 20,1 & 18,6 & 21,9 \\
\hline $\mathrm{R} 2$ & $54,2 \%$ & $45,8 \%$ & $15,2 \%$ & 18,9 & 14,9 & 25,8 \\
\hline R3 & $42,2 \%$ & $57,8 \%$ & $27,2 \%$ & 18,8 & 14,1 & 21,8 \\
\hline R4 & $52,0 \%$ & $48,0 \%$ & $31,4 \%$ & 19,0 & 13,5 & 25,6 \\
\hline R5 & $42,7 \%$ & $57,2 \%$ & $25,7 \%$ & 19,3 & 16,1 & 24,3 \\
\hline R6 & $35,7 \%$ & $64,3 \%$ & $30,4 \%$ & 19,5 & 17,0 & 23,4 \\
\hline R7 & $28,7 \%$ & $71,3 \%$ & $43,9 \%$ & 19,9 & 16,5 & 23,8 \\
\hline R8 & $0,1 \%$ & $99,9 \%$ & $93,6 \%$ & 22,2 & 18,7 & 25,5 \\
\hline R9 & $13,9 \%$ & $86,1 \%$ & $69,2 \%$ & 21,1 & 15,9 & 26,7 \\
\hline R10 & $33,4 \%$ & $66,6 \%$ & $24,1 \%$ & 19,4 & 15,8 & 21,9 \\
\hline R11 & $1,2 \%$ & $98,8 \%$ & $70,7 \%$ & 20,4 & 18,7 & 23,5 \\
\hline $\mathrm{R} 12^{*}$ & $15,1 \%$ & $84,9 \%$ & $69,3 \%$ & 20,8 & 16,3 & 23,9 \\
\hline R13* & $94,3 \%$ & $5,7 \%$ & $0,0 \%$ & 16,7 & 11,1 & 20,3 \\
\hline
\end{tabular}

*) Büroräume, keine Wohnnutzung

Tabelle 6.2: Statistische Betrachtung der gemessenen Raumlufttemperaturen während der Heizperiode 


\section{Messtechnische Untersuchung dünner Dämmschichten an massiven Wandkon- struktionen}

\subsection{Anheizphase einer massiven Wandkonstruktion unter Laborbedingungen}

Im Labor wurde das thermische Verhalten einer massiven Wandkonstruktion untersucht. Hierbei sind kontrollierte und wiederholbare thermische Bedingungen für die Wandkonstruktion hergestellt worden, so dass verschiedene Wandbekleidungen im Vergleich getestet werden konnten.

Der Versuchsaufbau soll dazu dienen, eine günstige Bemessung des Wandbelags zu ermitteln und eine versuchstechnische Überprüfung von gerechneten Werten vornehmen zu können.

Im Wohn- oder Büroraum sind natürlich weitere Einflüsse vorhanden, welche zum Beispiel durch die Raumlüftung und solare Gewinne durch die Verglasung verursacht werden.

Die Messergebnisse aus bewohnten Objekten sind in den Kapiteln 7.2 dargestellt.

Der Versuchsaufbau im Labor besteht aus zwei Klimakammern. Zwischen diesen beiden Klimakammern ist eine massive Versuchswand aus 17,5 cm Kalksandsteinmauerwerk mit der Rohdichte von $1600 \mathrm{~kg} / \mathrm{m}^{3}$ eingebaut, vergleiche Abbildung 7.1. Diese massive Wand ist thermisch zur Bodenplatte getrennt und die Wandenden sind oben und an den Seiten mit Dämmstoff belegt, so dass die Temperatureinflüsse aus der Umgebung für die Wandkonstruktion minimiert sind.

Die Wandoberflächentemperaturen sowie die Temperaturen in der Wand wurden im Mittelfeld der Wandansichtsfläche gemessen, um Einflüsse durch den Wand-Randbereich zu vermeiden.

Die Lufttemperatur ist mit einem Mantelthermoelement aus blankem Edelstahl gemessen worden, welches durch eine zusätzliche Schutzhülle aus $0,1 \mathrm{~mm}$ dicker Aluminiumfolie vor thermischer Strahlung geschützt wurde, vergleiche EN ISO 7726:2001 [41].

Die Oberflächentemperatur wurde mit einem Kontakt-Thermoelement ermittelt, das fest mit der Wandoberfläche verbunden (verklebt) war. Hierdurch wurde sichergestellt, dass sich keine Luftschicht zwischen dem Kontaktthermoelement und der Wandoberfläche befand oder bilden konnte. Gemessen und aufgezeichnet wurden die Raumluft- und Wandoberflächentemperaturen mit Thermoelementen des Typs T (Kupfer/Konstantan) und einem Datenlogger. Verwendet wurde hier ein 8-Kanal-Datenlogger der Firma RedLab, welcher über eine USB-Schnittstelle die Daten an den Rechner übermittelte. 
Die Temperaturkurven für die jeweilige Messstelle sind durch die Herstellersoftware der Datenlogger abgeglichen worden und die Thermoelemente waren für einen Bereich von $5^{\circ} \mathrm{C}$ bis $24^{\circ} \mathrm{C}$ kalibriert. Die Positionierung der Thermoelemente ist in Abbildung 7.2 dargestellt.
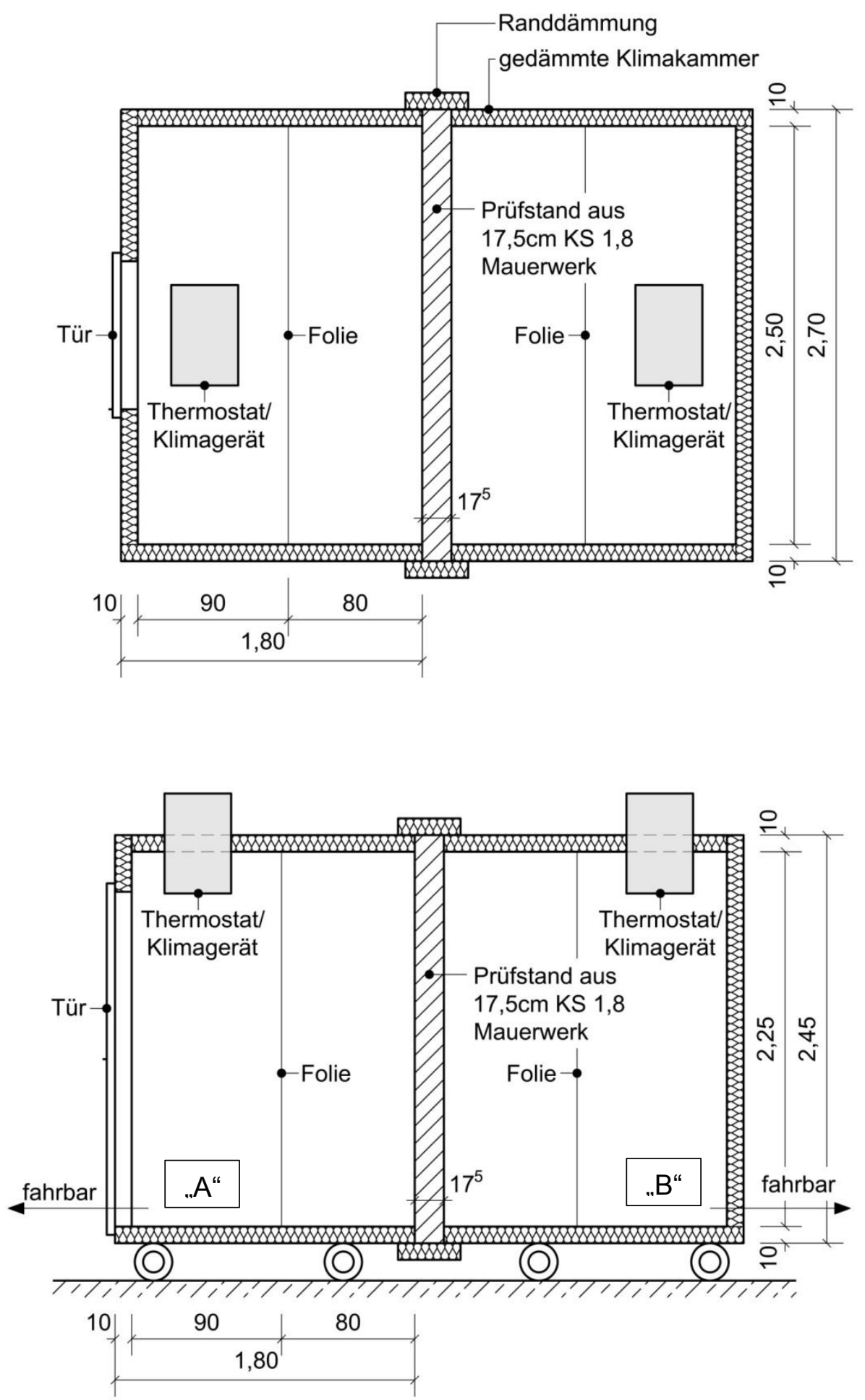

Abbildung 7.1: Grundriss- und Schnittzeichnung durch den Labor-Prüfstand 


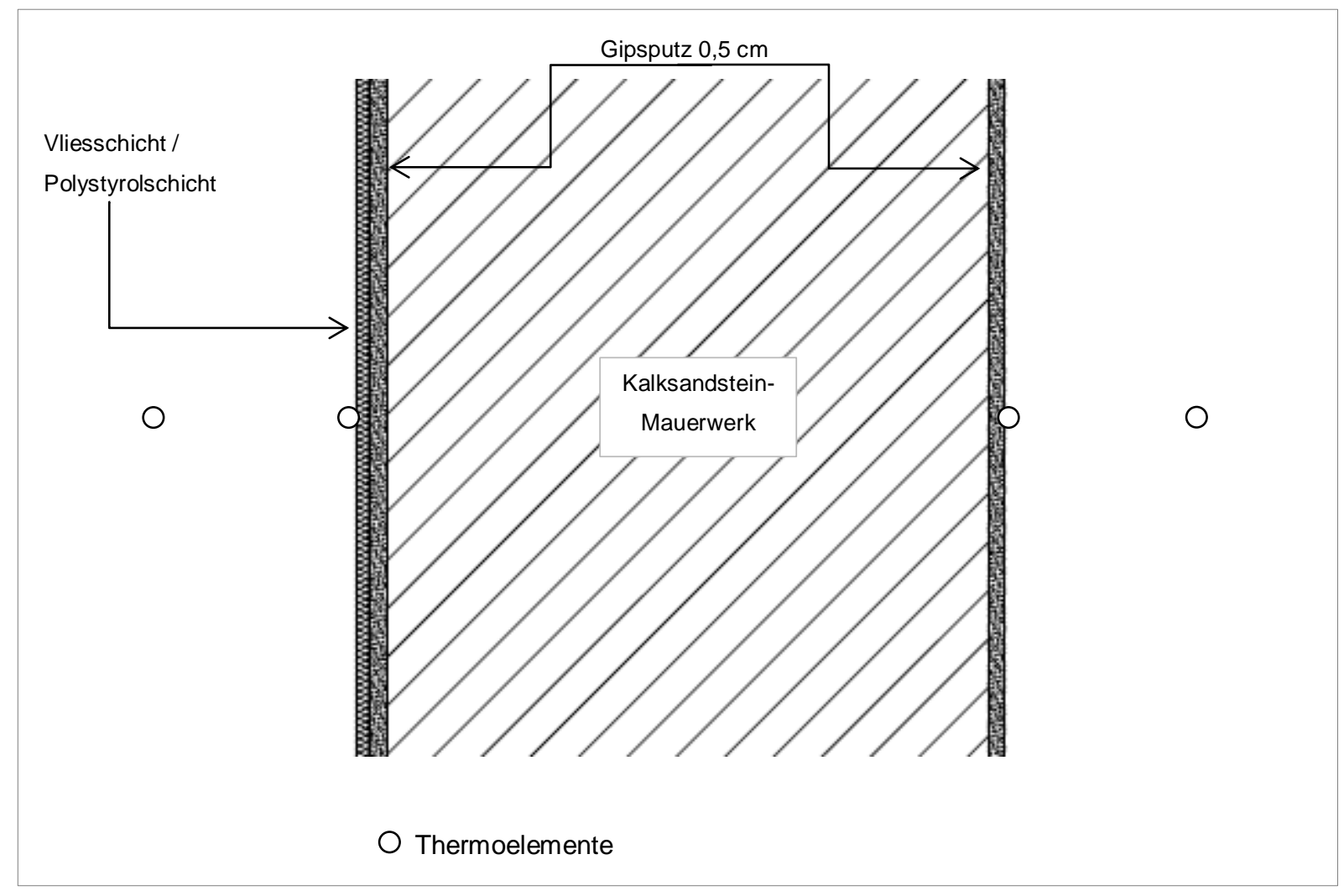

Abbildung 7.2: Schnittdarstellung der massiven Veruchswand inklusive der Positionierung der Thermoelemente

\begin{tabular}{|l|l|l|l|}
\hline Wandaufbau & Schichtdicke in $\mathbf{~}$ & Rohdichte $\left[\frac{\mathrm{kg}}{\mathrm{m}^{3}}\right]$ & Wärmeleitfähigkeit \\
\hline Gipsputz & 0,003 & 700 & $0,25^{2}$ \\
\hline KS-Mauerwerk & 0,175 & $1600^{3}$ & $0,79^{11}$ \\
\hline Gipsputz & 0,003 & 700 & $0,25^{2}$ \\
\hline Wandbelag & \multicolumn{2}{|c|}{ variabel } \\
\hline
\end{tabular}

1) nach DIN 4108-4, Tabellenwert

2) nach Herstellerangaben

3) gemessene mittlere Rohdichte (von mehreren Steinen)

Tabelle 7.1: thermische Eigenschaften der Veruchswand 
Die Beheizungszyklen sind mit verschiedenen Wandbeschichtungen - ohne und bis zu $4 \mathrm{~mm}$ Filz und $30 \mathrm{~mm}$ Polystyrol auf einer Prüfwandseite - bei unterschiedlichen thermischen Bedingungen gemessen worden, vergleiche Tabelle 7.2.

Die Wandbeschichtungen wurden direkt auf den oben beschriebenen Wandaufbau vollflächig verklebt, so dass keine Luftspalten zwischen der Beschichtung und dem Wandaufbau auftreten konnten.

\begin{tabular}{|c|c|c|c|c|}
\hline & \multicolumn{3}{|c|}{ Wandbeschichtung auf der Prüfwand in der Kammer A } \\
\hline Versuch & $\begin{array}{c}\text { Schichtstärke in } \\
\mathbf{m m}\end{array}$ & $\begin{array}{c}\text { Material } \\
{[-]}\end{array}$ & $\begin{array}{c}\text { Rohdichte } \\
\mathbf{k g}\end{array}$ & $\begin{array}{c}\mathbf{\lambda} \text { Wärmeleitfähigkeit } \\
\mathbf{W}\left(\mathbf{m}^{\star} \mathbf{K}\right)\end{array}$ \\
\hline $\mathbf{\mathbf { m } ^ { \mathbf { 3 } }}$ & - & - & - & - \\
\hline $\mathbf{1}$ & 1,2 & Vliesschicht & 250 & 0,04 \\
\hline $\mathbf{2}$ & 2,4 & Vliesschicht & 250 & 0,04 \\
\hline $\mathbf{3}$ & 3,6 & Vliesschicht & 250 & 0,04 \\
\hline
\end{tabular}

Tabelle 7.2: Untersuchte Wandbeschichtung bei den Versuchen im Prüfstand in der Kammer A 


\subsubsection{Anheizphase im Labor-Prüfstand für eine einmalige Anheizung}

Im Labor-Prüfstand (Hot-Box) ist eine Temperaturanhebung auf die operative Temperatur von $12^{\circ} \mathrm{C}$ auf $21^{\circ} \mathrm{C}$ durchgeführt worden, vergleiche Abbildung 7.3. Die Ergebnisse zeigen die Entwicklung der Oberflächentemperaturen in einer Anheizphase mit unterschiedlichen Wandbeschichtungen. In der Tabelle 7.3 sind die Zeiten bis zum Erreichen der operativen Temperatur von $19,0^{\circ} \mathrm{C}, 19,5^{\circ} \mathrm{C}$ und $20^{\circ} \mathrm{C}$ mit den unterschiedlichen Wandoberflächenmaterialien dargestellt.

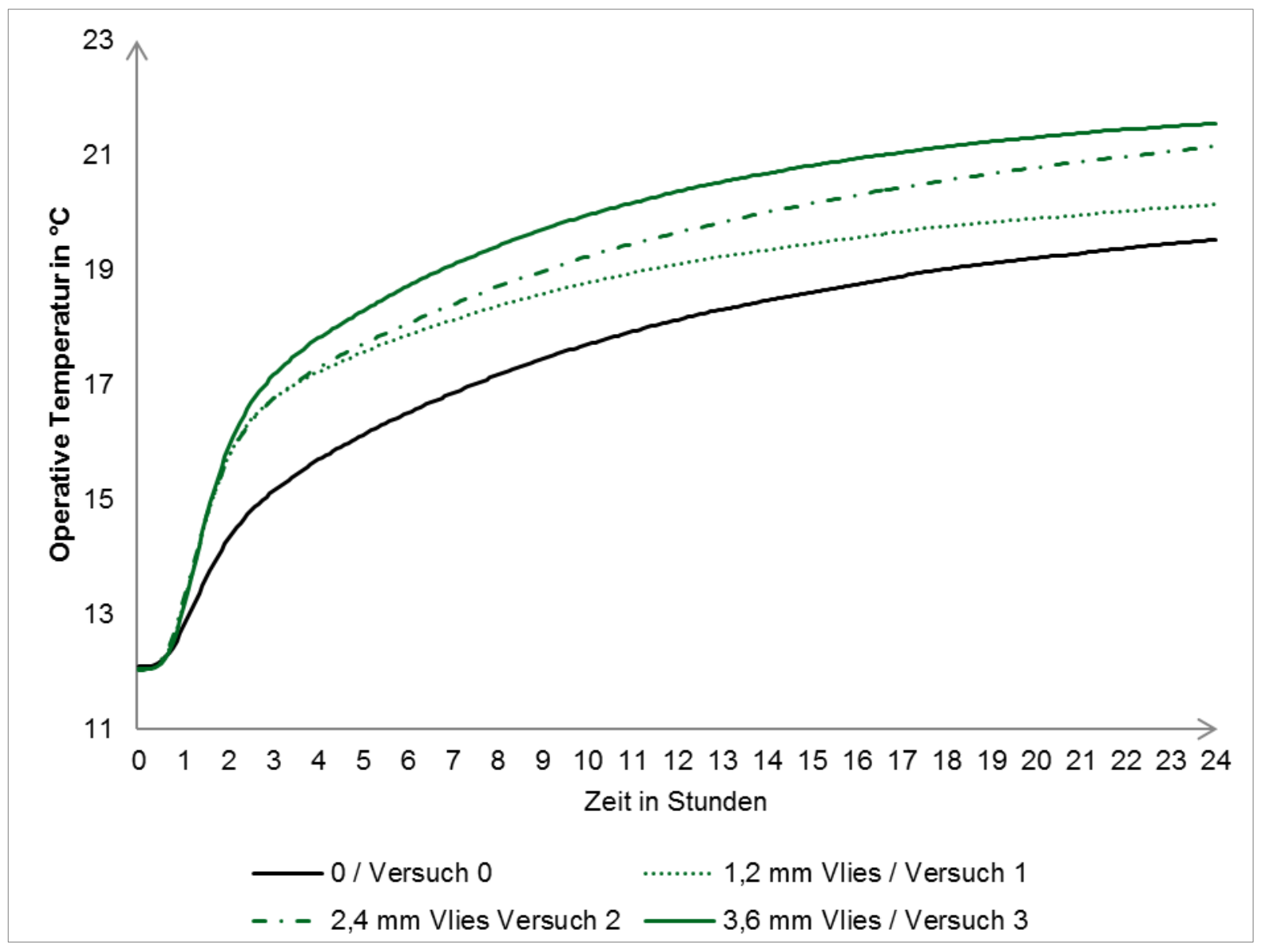

Abbildung 7.3: Zeitliche Oberflächentemperaturentwicklungen auf der Prüfwand (in Kammer A) bei verschiedenen Beschichtungen und bei einem einmaligen Anheizvorgang

\begin{tabular}{|l|c|c|c|}
\hline \multirow{2}{*}{ Versuch } & \multicolumn{3}{|c|}{ Zeitspanne bis zum Erreichen operative Temperatur von } \\
\cline { 2 - 4 } & $\mathbf{1 9 , 0 ^ { \circ } \mathbf { C }}$ & $\mathbf{1 9 , 5 ^ { \circ } \mathbf { C }}$ & $\mathbf{2 0 , 0 ^ { \circ } \mathbf { C }}$ \\
\hline $0 /$ ohne Belag & $17,9 \mathrm{~h}$ & $23,8 \mathrm{~h}$ & $21,7 \mathrm{~h}$ \\
\hline $1 / 1,2 \mathrm{~mm}$ Vliesschicht & $11,4 \mathrm{~h}$ & $15,5 \mathrm{~h}$ & $14,0 \mathrm{~h}$ \\
\hline $2 / 2,4 \mathrm{~mm}$ Vliesschicht & $9,1 \mathrm{~h}$ & $11,3 \mathrm{~h}$ & $10,3 \mathrm{~h}$ \\
\hline $3 / 3,6 \mathrm{~mm}$ Vliesschicht & $6,7 \mathrm{~h}$ & $8,3 \mathrm{~h}$ & $2,6 \mathrm{~h}$ \\
\hline $4 / 30 \mathrm{~mm}$ Polystyrol & $1,9 \mathrm{~h}$ & $2,2 \mathrm{~h}$ & \\
\hline
\end{tabular}

Tabelle 7.3: Dauer bis zum Erreichen der jeweiligen operativen Temperatur von $19,0^{\circ} \mathrm{C}, 19,5^{\circ} \mathrm{C}$ und $20^{\circ} \mathrm{C}$ 


\subsubsection{Anheizphase im Labor-Prüfstand bei einer intermittierenden Beheizung}

Im Labor-Prüfstand (Hot-Box) ist eine intermittierende Beheizung über Raumluft-Temperaturveränderungen zwischen $21^{\circ} \mathrm{C}$ auf $17^{\circ} \mathrm{C}$ in mehreren Zyklen durchgeführt worden, bis ein eingeschwungener Zustand vorlag. In Abbildung 7.4 ist der eingeschwungene Zustand dargestellt. Aus Tabelle 7.4 sind die Zeiten bis zum Erreichen der operativen Temperatur von $19,0^{\circ} \mathrm{C}, 19,5^{\circ} \mathrm{C}$ und $20^{\circ} \mathrm{C}$ unter dem Einfluss verschiedener Wandbeschichtungen zu entnehmen.

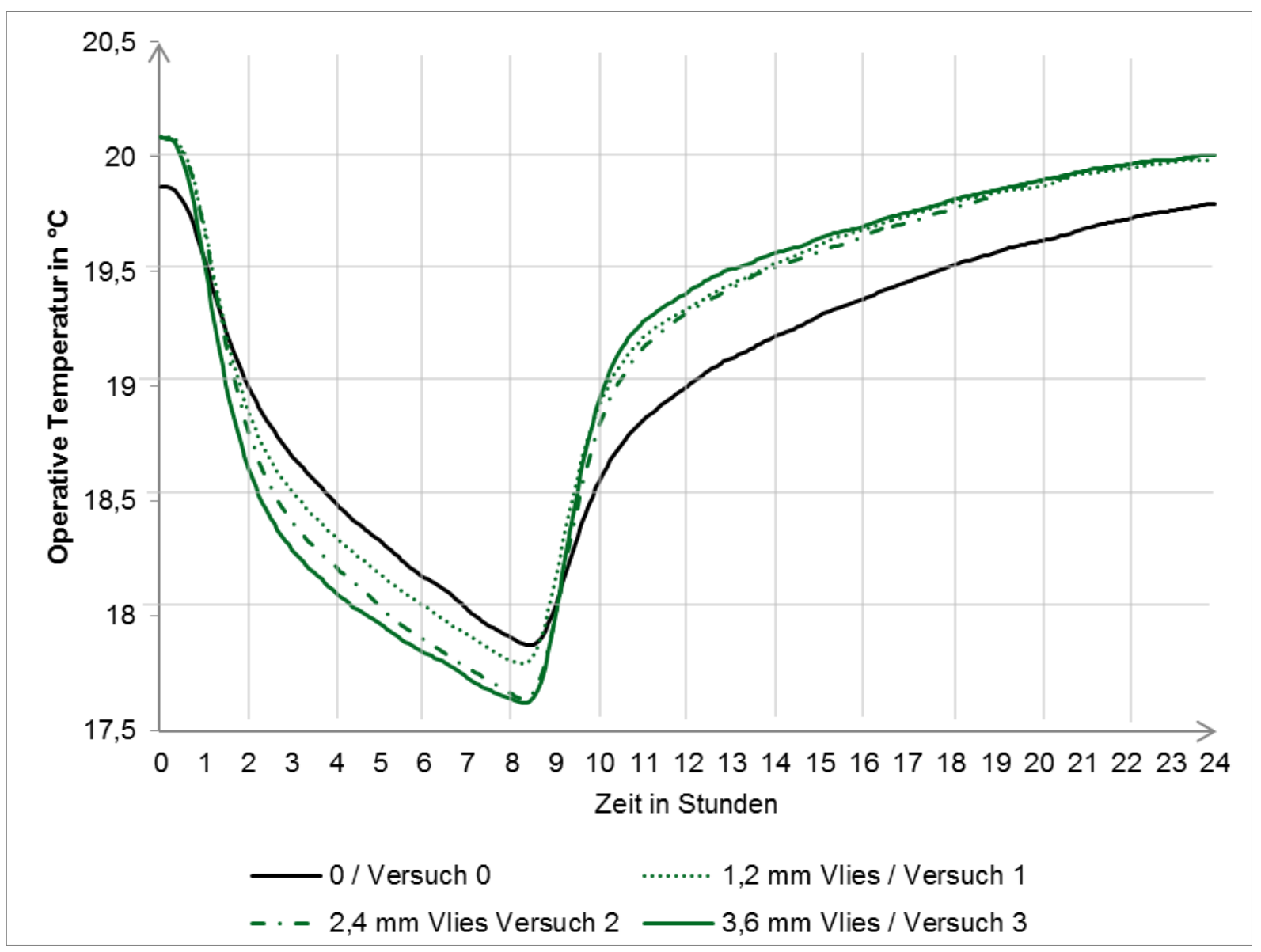

Abbildung 7.4: Zeitliche Oberflächentemperaturentwicklungen auf der Prüfwand (in Kammer „A“) bei verschiedenen Beschichtungen und einer intermittierenden Beheizung

\begin{tabular}{|l|c|c|c|}
\hline \multirow{2}{*}{ Wandbeschichtungen } & \multicolumn{3}{|c|}{ Zeitspanne bis zum Erreichen operative Temperatur von } \\
\cline { 2 - 4 } & $\mathbf{1 9 , 0 ^ { \circ } \mathbf { C }}$ & $\mathbf{1 9 , 5 ^ { \circ } \mathbf { C }}$ & $\mathbf{2 0 , 0}^{\circ} \mathbf{C}$ \\
\hline 0 / ohne Belag & $3,6 \mathrm{~h}$ & $9,3 \mathrm{~h}$ & wird nicht erreicht \\
\hline $1 / 1,2 \mathrm{~mm}$ Vliesschicht & $1,8 \mathrm{~h}$ & $5,3 \mathrm{~h}$ & $15,6 \mathrm{~h}$ \\
\hline $2 / 2,4 \mathrm{~mm}$ Vliesschicht & $2,0 \mathrm{~h}$ & $5,3 \mathrm{~h}, 2 \mathrm{~h}$ \\
\hline $3 / 3,6 \mathrm{~mm}$ Vliesschicht & $1,6 \mathrm{~h}$ & $4,4 \mathrm{~h}$ & $15,1 \mathrm{~h}$ \\
\hline $4 / 30 \mathrm{~mm}$ Polystyrol & $1,4 \mathrm{~h}$ & $1,9 \mathrm{~h}$ & $9,6 \mathrm{~h}$ \\
\hline
\end{tabular}

Tabelle 7.4: Dauer bis zum Erreichen der jeweiligen operativen Temperatur von $19,0^{\circ} \mathrm{C}, 19,5^{\circ} \mathrm{C}$ und $20^{\circ} \mathrm{C}$ 


\subsection{Anheizphase in einem Wohngebäude}

In einem Wohngebäude sind zwei Räume mit gleichen geometrischen und konstruktiven Bedingungen einschließlich der Beheizung, Fenstergröße und Fensterausrichtung untersucht worden. Die betrachteten Räume sind vorwiegend - bis auf die Deckenkonstruktion - in Massivbauweise erstellt. Einer dieser Räume wurde mit einer $4 \mathrm{~mm}$ dicken Vliesschicht an den Innen- und Außenwänden versehen, vergleiche Abbildung 7.5.

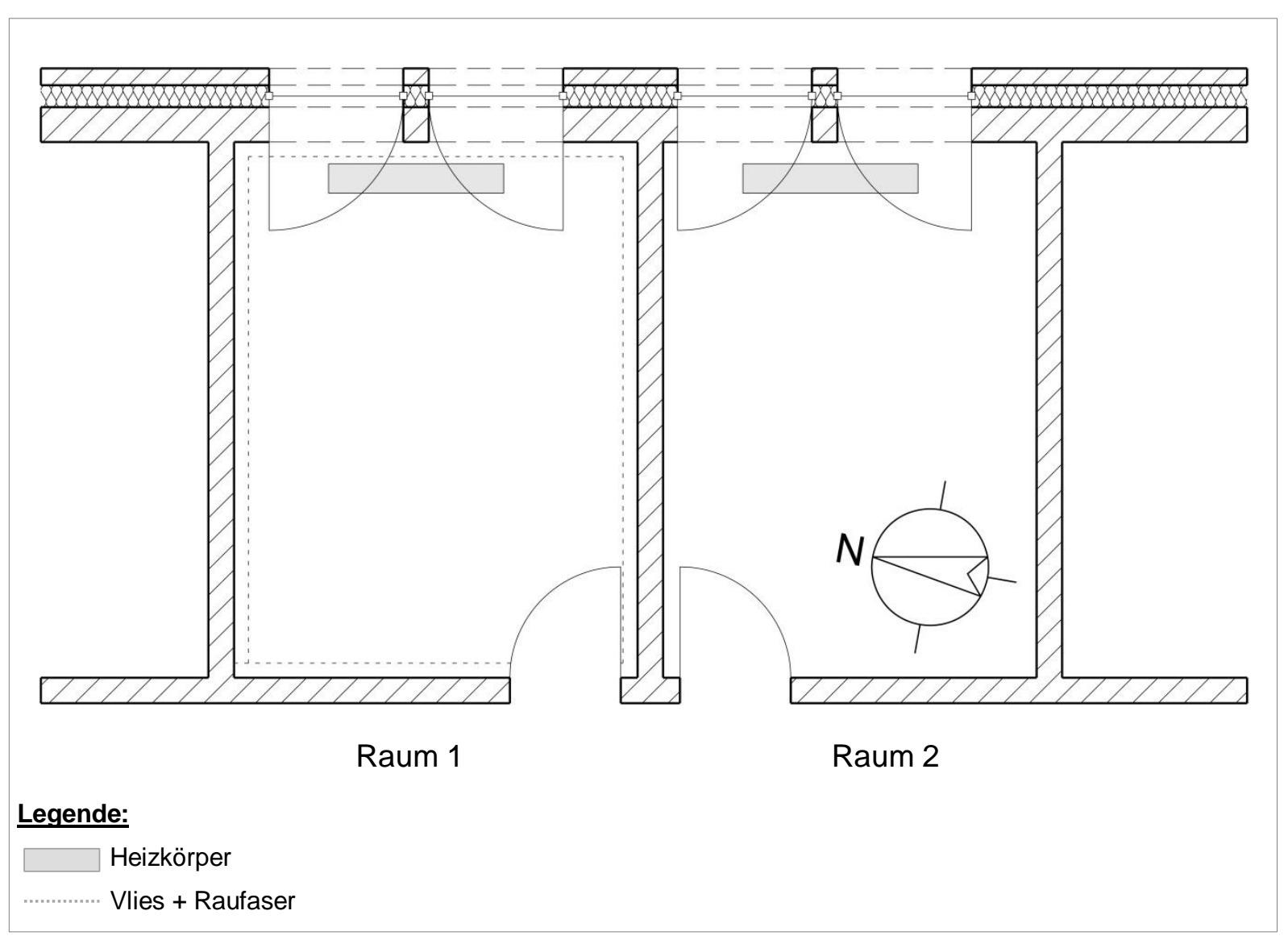

Abbildung 7.5: Darstellung der beiden Versuchsräume in einem bewohnten Objekt 


\begin{tabular}{|l|c|}
\hline Bauteil & Material \\
\hline Außenwand & $12,5 \mathrm{~cm}$ Kalksandstein-Mauerwerk \\
& $\sim 9 \mathrm{~cm}$ Schalenabstand gefüllt mit Perlite \\
$10,5 \mathrm{~cm}$ Verblendmauerwerk
\end{tabular}

Tabelle 7.5: Konstruktionsdaten der beiden Versuchsräume

Nach einer Nachtabsenkung der beiden Räume auf eine Ausgangsraumlufttemperatur von $17^{\circ} \mathrm{C}$ zeigt sich, dass der Raum mit der Vliesschicht an den Wänden gegenüber dem Raum ohne Vliesschicht an den Wänden eine deutlich kürzere Raumlufttemperaturanheizphase besitzt, vergleiche Abbildung 7.6. 


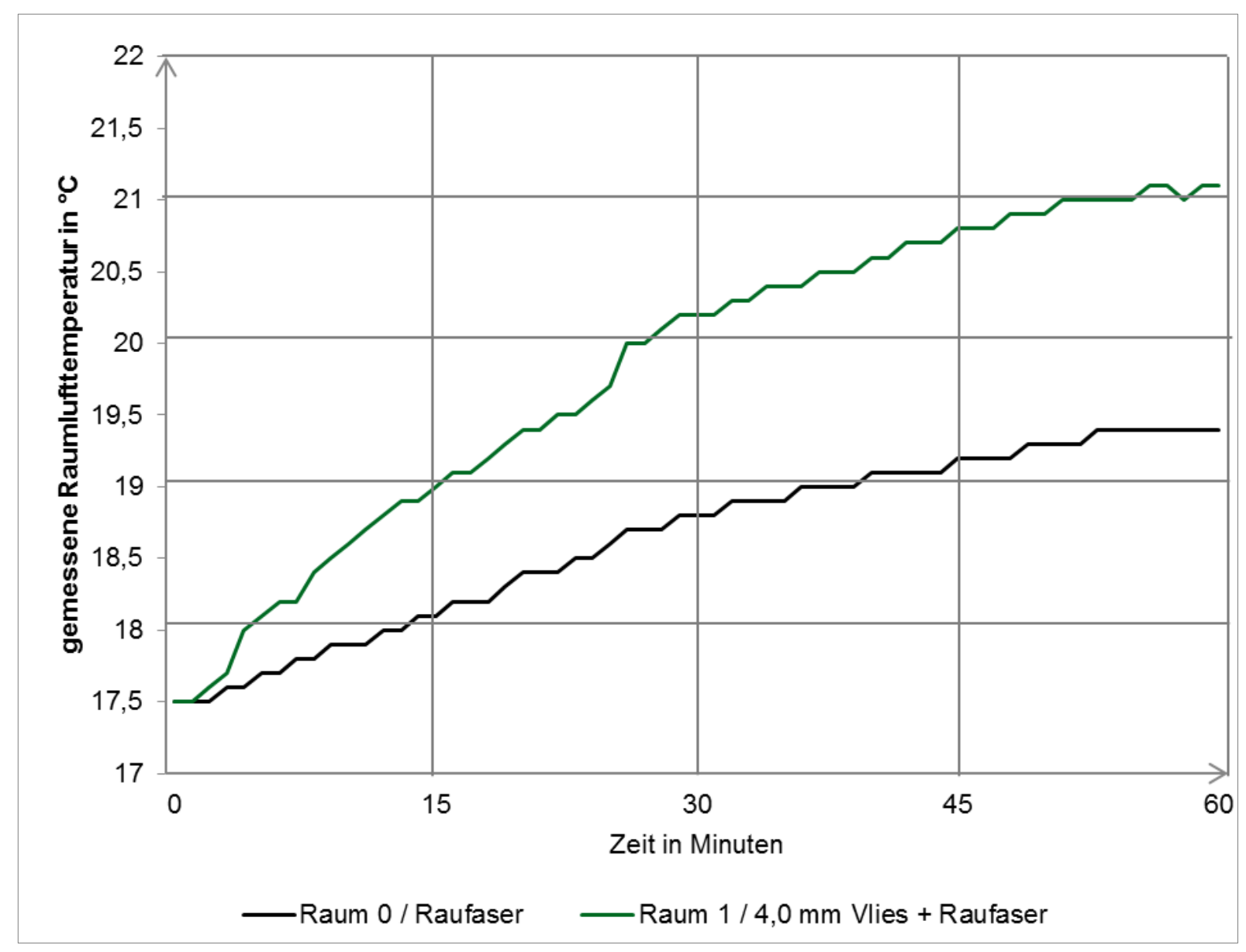

Abbildung 7.6: Zeitliche Raumlufttemperaturentwicklung während einer Anheizphase in zwei gleichen Räumen ohne und mit einer Wandbeschichtung aus $4,0 \mathrm{~mm}$ Vliesschicht

In Tabelle 7.6 sind die Zeiten bis zur Erzielung der Raumlufttemperatur von $19,0^{\circ} \mathrm{C}, 19,5^{\circ} \mathrm{C}$ und $20^{\circ} \mathrm{C}$ dargestellt.

Wie bei den Laborversuchen zeigt sich auch in einem realen Raum mit einer entsprechenden Vliesschicht an den Wänden eine erheblich kürzere Anheizzeit gegenüber der Situation in einem Raum ohne Vliesschicht. Für die hier untersuchten Raumsituationen ergibt sich mit einer Vliesbeschichtung mehr als eine Halbierung der Anheizphase.

\begin{tabular}{|l|c|c|c|}
\hline \multirow{2}{*}{ Wandoberfläche } & \multicolumn{3}{|c|}{ Zeitspanne bis zum Erreichen der jeweiligen Raumlufttemperatur } \\
\cline { 2 - 4 } & $\mathbf{1 9 , 0 ^ { \circ } \mathbf { C }}$ & $\mathbf{1 9 , 5 ^ { \circ } \mathbf { C }}$ & $\mathbf{2 0 , 0}^{\circ} \mathbf{C}$ \\
\hline 0 / ohne Wandbeschichtung & $36 \mathrm{~min}$ & $61 \mathrm{~min}$ & $>60 \mathrm{~min}$ \\
\hline $1 /$ mit $4,0 \mathrm{~mm}$ Vliesschicht & $15 \mathrm{~min}$ & $22 \mathrm{~min}$ & $26 \mathrm{~min}$ \\
\hline
\end{tabular}

Tabelle 7.6: Dauer bis zum Erreichen der jeweiligen Raumlufttemperatur von $19,0^{\circ} \mathrm{C}, 19,5^{\circ} \mathrm{C}$ und $20^{\circ} \mathrm{C}$ 
Das Temperaturfeld in der Innenwandkonstruktion des hier untersuchten Objekts wurde mit Hilfe der Software „HeatTransmission" berechnet und ist in Abbildung 7.7 dargestellt. Der berechnete Temperaturverlauf über die Anheizphase zeigt einen deutlichen Unterschied zwischen der Wandoberfläche mit der dünnen Dämmschicht von 4,0 mm, (hier links) und der Wandoberfläche ohne Dämmschicht (hier rechts).

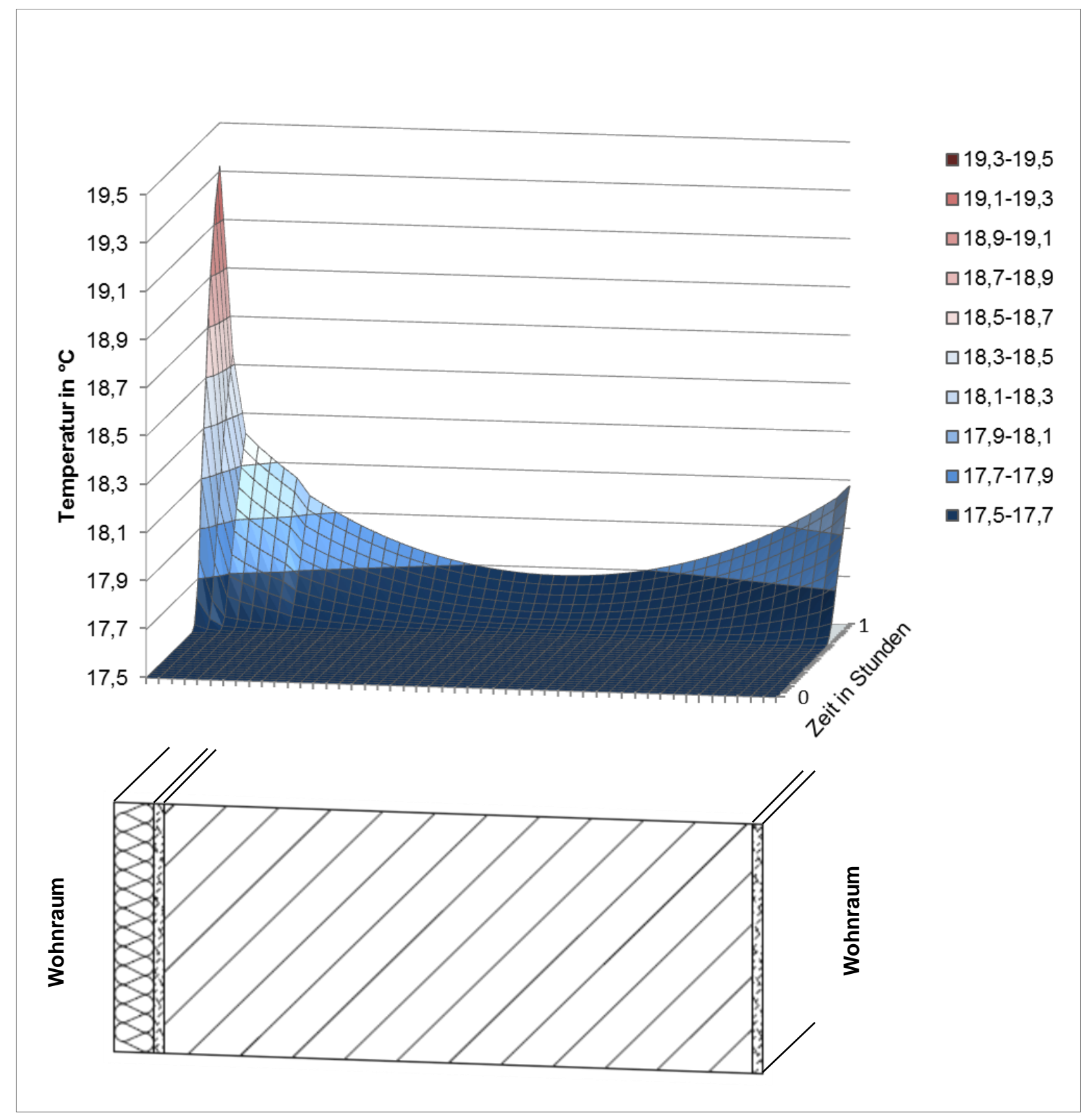

Abbildung 7.7: Gerechneter Temperaturverlauf über den Innenwandquerschnitt und in der zeitlichen Entwicklung für das untersuchte Objekt bei einer Vliesschicht von $4,0 \mathrm{~mm}$ 


\subsection{Einfluss von solarer Strahlung auf eine Innenwand}

Es wurde ein $11,5 \mathrm{~cm}$ dickes Kalksandstein-Mauerwerk in einem unbeheizten Raum mit einem Abstand von vier Metern hinter einem Fensterband errichtet. Der Versuch zeigt, wie sich die Speicherfähigkeit aufgrund einer dünnen wärmedämmenden Vliesschicht gegenüber einer unbeschichteten Wand verändert.

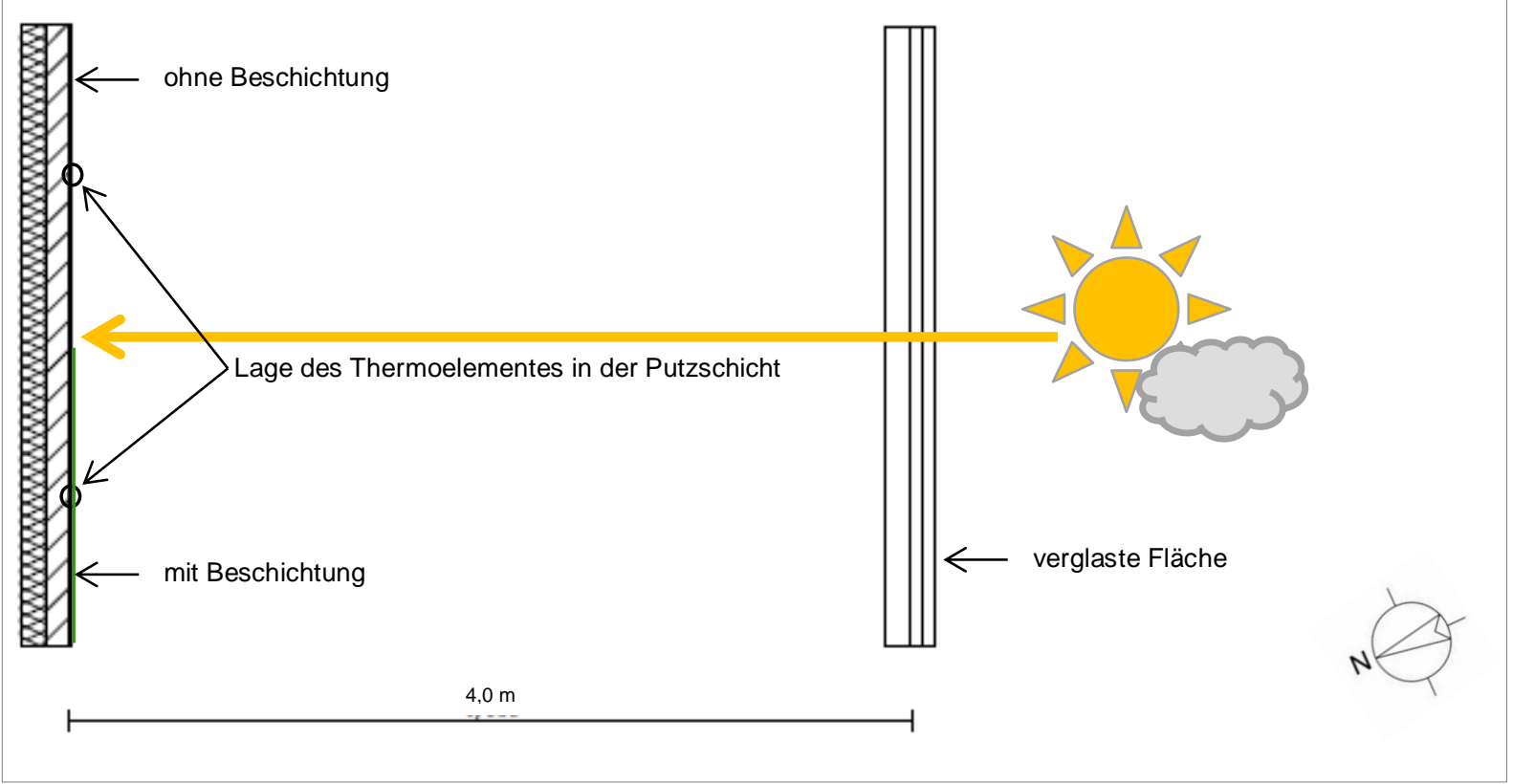

Abbildung 7.8: Schematische Darstellung der Wandkonstruktion im Grundriss

Damit der Temperaturverlauf nicht durch andere Einflüsse gestört wurde, ist eine Wärmedämmschicht mit $80 \mathrm{~mm}$ Dicke auf der Rückseite der Veruchswand aufgebracht worden, vergleiche Abbildung 7.8 und 7.9. 


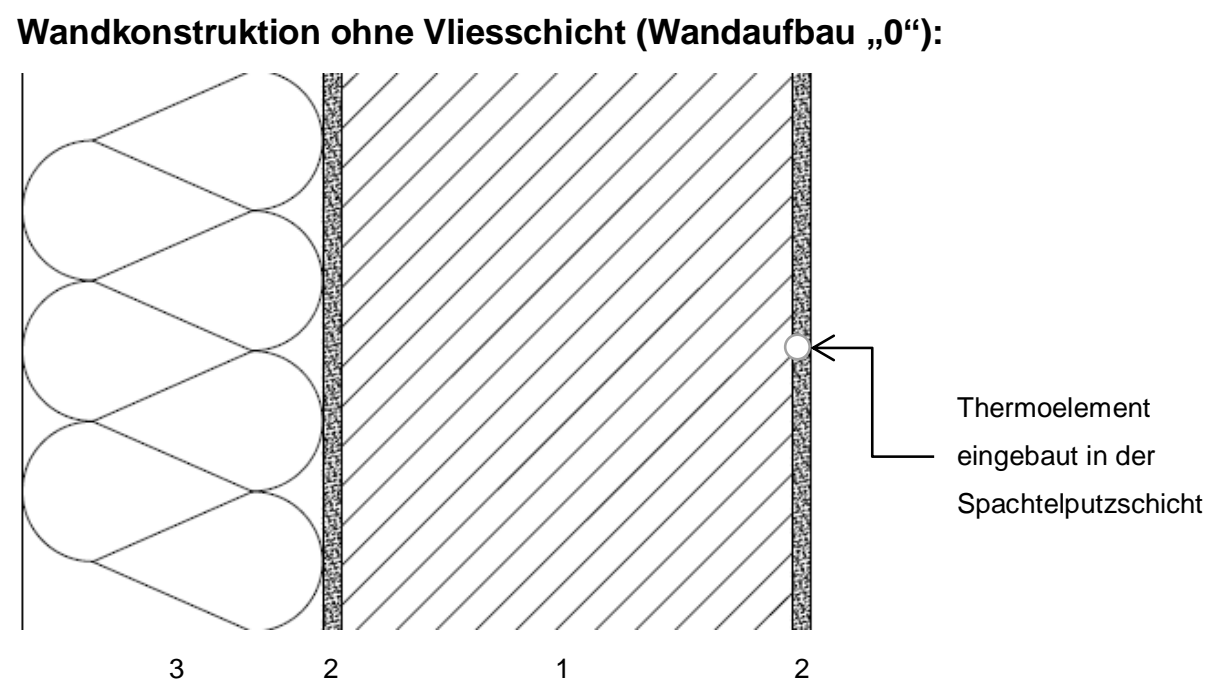

Wandkonstruktion mit Vliesschicht (Wandaufbau „1“):

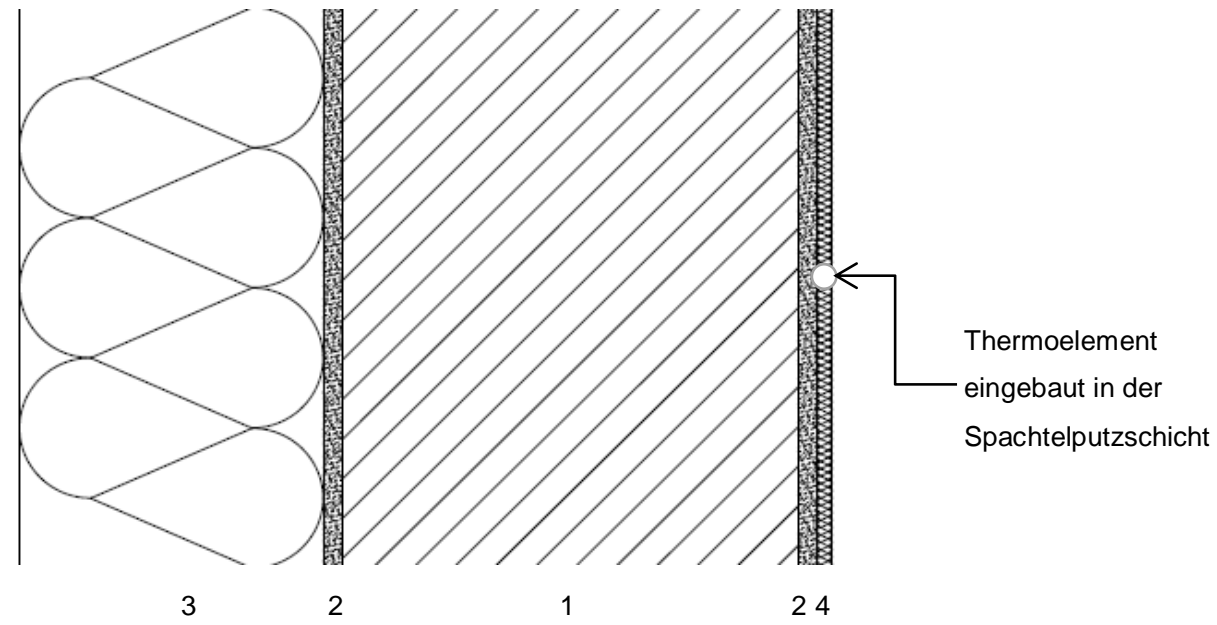

Bauteilschichten:

1) $115 \mathrm{~mm}$ Kalksandstein-Mauerwerk

2) $5 \mathrm{~mm}$ Putzschicht

3) $80 \mathrm{~mm}$ Wärmedämmung, Mineralwolle

4) $4 \mathrm{~mm}$ Vliesschicht

Abbildung 7.9: Wandkonstruktionen ohne und mit Vliesschicht 
Die Messungen an der beschriebenen Versuchswand wurden in der Herbstzeit in einem nicht beheizten Raum durchgeführt.

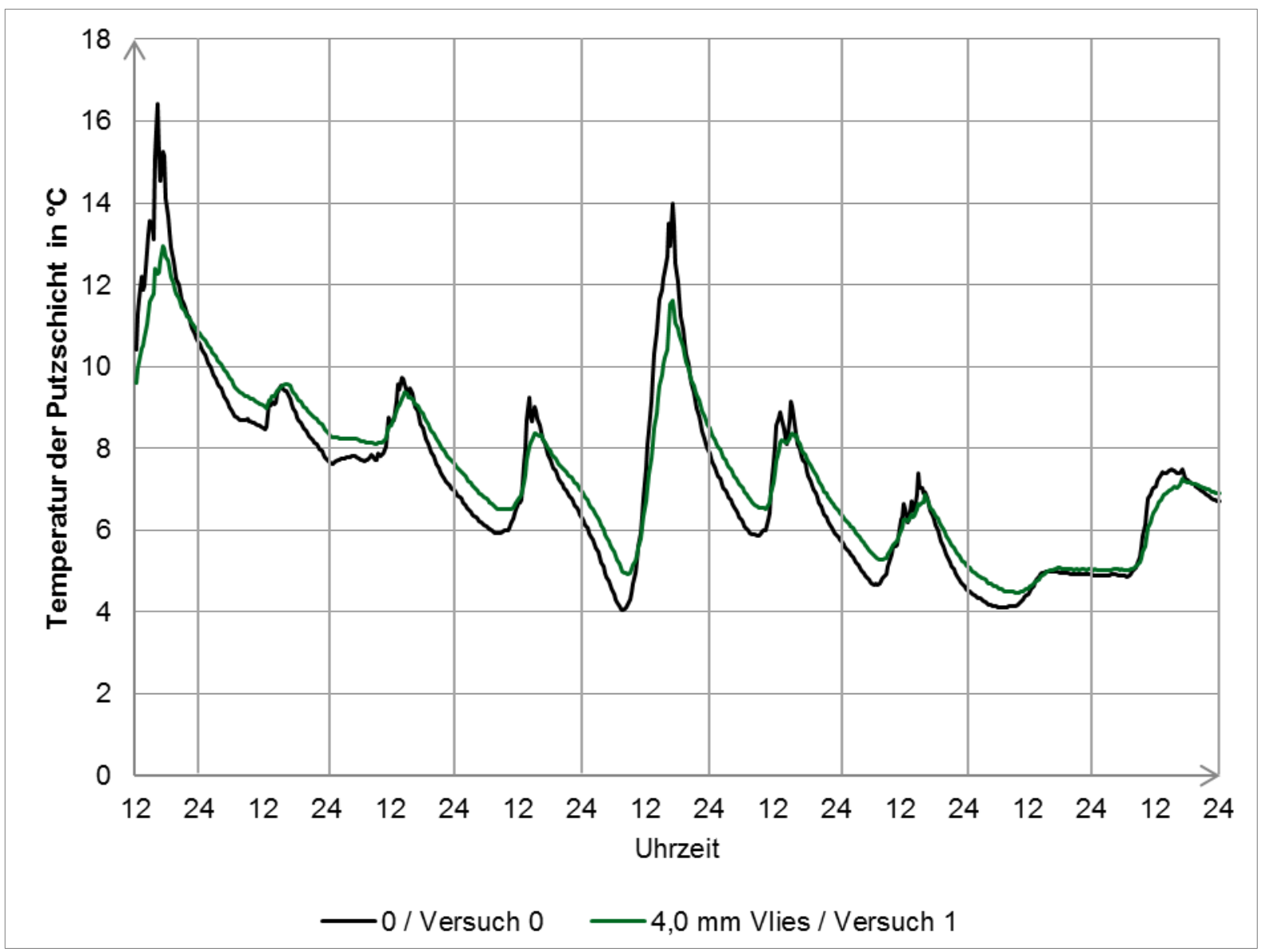

Abbildung 7.10: Einfluss durch solare Strahlung auf eine Massivwand ohne und mit einer dünnen Vliesschicht

Abbildung 7.10 zeigt den thermischen Einfluss, welcher durch eine dünne Vliesschicht (4 $\mathrm{mm}$ ) bei einer solaren Einstrahlung gegenüber einer normalen Wandsituation entsteht.

Bei der Ausführung mit einer Vliesschicht ist gegenüber der normalen Wandsituation eine Temperaturbedämpfung zu erkennen. Hierdurch sind zwar die Temperaturspitzenwerte durch solare Einstrahlung mit einer Vliesbeschichtung geringer, jedoch die mittlere Temperatur - durch eine effektiverer Wärmespeicherung - für eine solare Energienutzung günstiger aus. 


\subsection{Lüftungseinfluss auf die operative Temperatur}

Messtechnisch wurde die Raumauskühlung während einer Fensterlüftung untersucht. Der Versuch ist in zwei gleichen Versuchsräumen durchgeführt wurden. Die beiden Räume besitzen folgende identische geometrische und konstruktive Randbedingungen:

$\begin{array}{ll}\text { Grundfläche: } & \begin{array}{l}22 \mathrm{~m}^{2} \\ \text { Raumhöhe: }\end{array} \\ \begin{array}{l}\text { Fensteröffnung: } \\ \text { Türöffnung: }\end{array} & \begin{array}{l}\text { keine vorhanden } \\ 1,0 \mathrm{~m} \times 2,0 \mathrm{~m}\end{array} \\ \text { Wände: } & 17,5 \mathrm{~cm} \text { Kalksandsteinmauerwerk mit Gipsputzschicht } \\ \text { Decke: } & 20 \mathrm{~cm} \text { Normalbetondecke } \\ \text { Boden: } & 14 \mathrm{~cm} \text { Normalbetondecke ohne Estrich } \\ & \text { Ölradiator mit 2000 W } \\ \text { Beheizung: } & \text { ohne Wärmedämmschichten } \\ \text { Dämmung der Wände: } & 10 \mathrm{~cm} \text { Mineralfaserdämmung } \\ \text { Decke und Boden: } & \end{array}$

Die Wände im Versuchsraum 1 wurden mit einer $4 \mathrm{~mm}$ starken Vliesschicht beklebt. Beide Räume sind mit einer Raumlufttemperatur von $21^{\circ} \mathrm{C}$ über einen Zeitraum von sieben Tagen beheizt worden. Anschließend wurde die Beheizung der beiden Räume jeweils für einen Zeitraum von vier Stunden unterbrochen. Nach der Unterbrechung erfolgte eine 15-minütige Lüftung über die geöffnete Türe in einen kühlen Hallenraum. Die einströmende Luft hatte während der Lüftung eine Temperatur von $2^{\circ} \mathrm{C}$. Nach der Lüftungsphase wurden die Türen wieder verschlossen und die Beheizung fortgesetzt.

In Abbildung 7.11 sind die operativen Temperaturen während der einzelnen TemperaturPhasen dargestellt. Es ist zu erkennen, dass Versuchsraum 1 mit einer $4 \mathrm{~mm}$ dicken Vliesschicht auf den Wänden deutlich schneller nach der Belüftungsphase anheizbar war. Auffällig ist, dass die operativen Temperaturen zu Beginn der Beheizung im Versuchsraum 1 höher waren als im Versuchsraum 0. Der Grund hierfür ist im Wandbeschichtungsunterschied zu sehen. Der Energieabfluss ist ohne Vliesschicht größer, so dass die Oberflächentemperaturen und die Raumlufttemperaturen in Versuchsraum 0 niedriger blieben. 


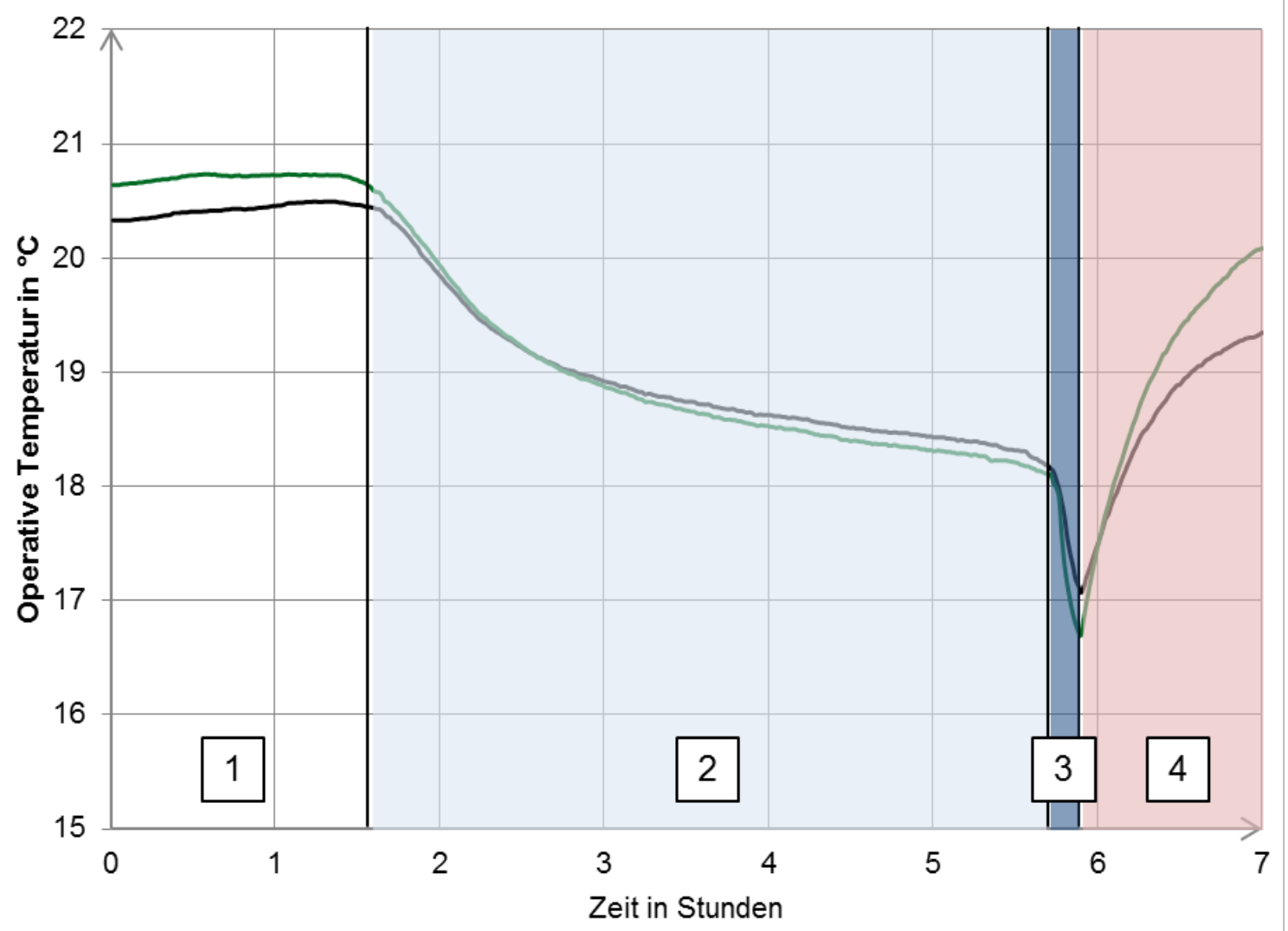

—Versuchsraum $0 \quad$ Versuchsraum $1 \mathrm{mit} 4 \mathrm{~mm}$ Vlies auf den Wänden

1 Beheizungsphase

2 Heizungsunterbrechung für 4 Stunden

3 15-minütigen Lüftung bei $2^{\circ} \mathrm{C}$ Außentemperatur

$4 \quad$ Wiederanheizung nach der Lüftung

Abbildung 7.11: Operative Temperaturen während unterschiedlicher thermischer Einwirkungen auf zwei gleichartige Räume mit und ohne Vliesbeschichtung an den Wänden 


\subsection{Energieeinsparung bei einem Wohngebäude}

Das mögliche Energieeinsparpotenzial durch Anbringung einer Vliesschicht an den raumseitigen Wandoberflächen wurde in einem bewohnten Objekt überprüft. Hierzu wurde eine 4,0 mm dicke Vliesschicht an den Innen- und Außenwänden in Räumen mit Nachtspeicherheizungen eingebaut. In diesem Objekt, welches um 1900 erbaut wurde, sind sonst keine weiteren Veränderungen vorgenommen worden. Der Wärmeschutz entspricht bis auf die Verbesserung der Fenster im Jahr 1975 den Bedingungen aus dem Jahr 1900.

\section{Objektdaten:}

$\begin{array}{ll}\text { Haustyp: } & \text { Bauernhof, freistehend } \\ \text { Baujahr: } & \sim 1900 \\ \text { Modernisierung: } & \sim 1975 \\ \text { Fenster: } & \text { Isolierverglasung (ab 1975) } \\ \text { Außenwände: } & \text { Ziegelmauerwerk } \\ \text { Wohnfläche: } & 114 \mathrm{~m}^{2} \\ \text { Beheizte Wohnfläche: } & 33 \mathrm{~m}^{2} \\ \text { Art der Beheizung: } & \text { Nachtspeicherheizung } \\ \text { Wohnsituation: } & 2-\text { Personenhaushalt, kontinuierliche Beheizung ohne } \\ & \text { echte Nachtunterbrechung - wegen der Nachtspeicherheizung }\end{array}$

Raumseitig wurde an den Innen- und Außenwänden am 11.12.2008 eine dünne Dämmschicht (4,0 mm Vliesschicht) angebracht. Die Jahresenergieverbräuche sind in Tabelle 7.8 dargestellt. Die Monatsmitteltemperaturen sind für die Heizperiode angegeben, um die klimatischen Bedingungen mit den Verbrauchsdaten vergleichen zu können. Die Monatsmitteltemperaturen waren in den Heizperioden 2008 - 2010 deutlich niedriger als in den Heizperioden 2006 - 2008. Dennoch ist hier eine hohe Energieeinsparung gegenüber den Heizperioden 2006 - 2008 ab dem 11.12.2008 erzielt worden, vergleiche Tabelle 7.8.

Neben der Energieeinsparung hat sich die thermische Behaglichkeit wesentlich verbessert. Die Oberflächentemperaturen sowie die Anheizbarkeit sind deutlich günstiger geworden. 
Grundriss vom beheizten Teil des Wohngebäudes:

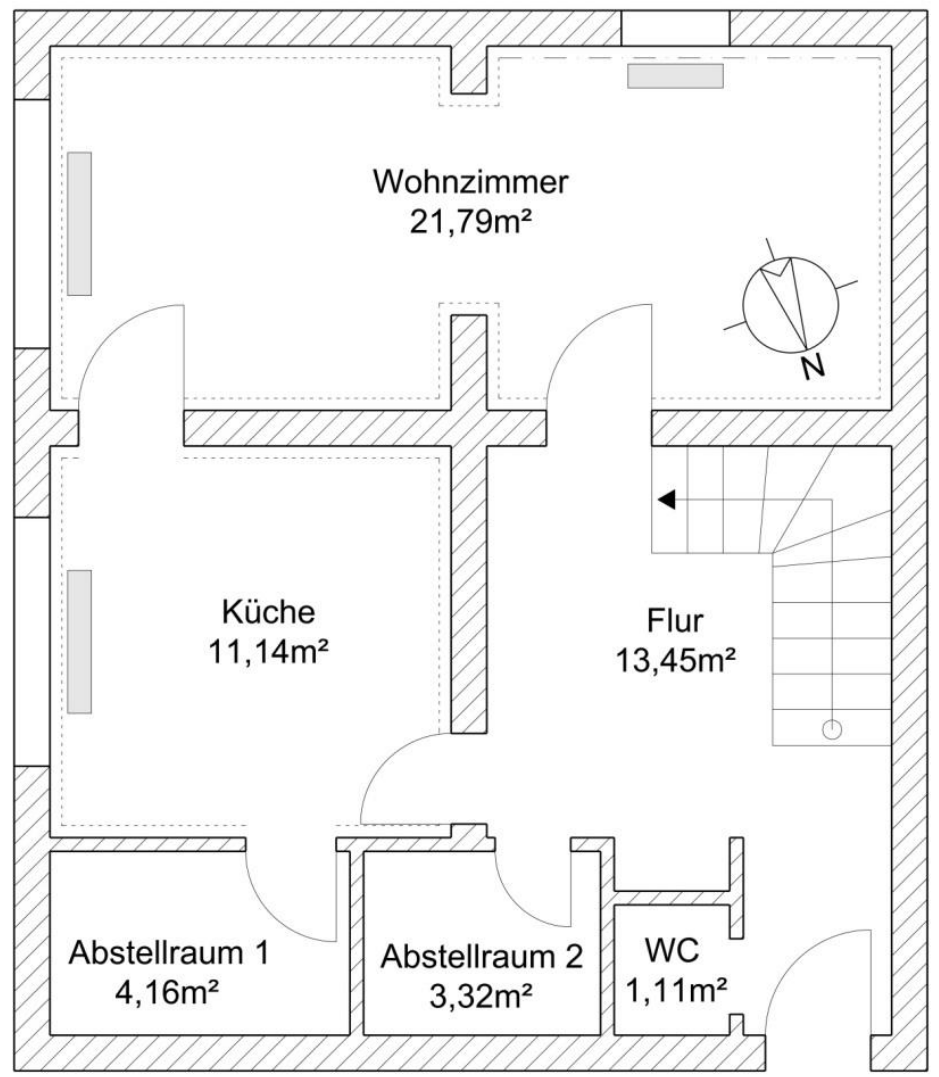

Legende:

Nachtspeicher

4,0 mm Vlies + Raufaser

- - Holzverkleidung

Abbildung 7.12: Erdgeschoss-Grundriss des untersuchten Wohngebäudes aus dem Baujahr 1900 


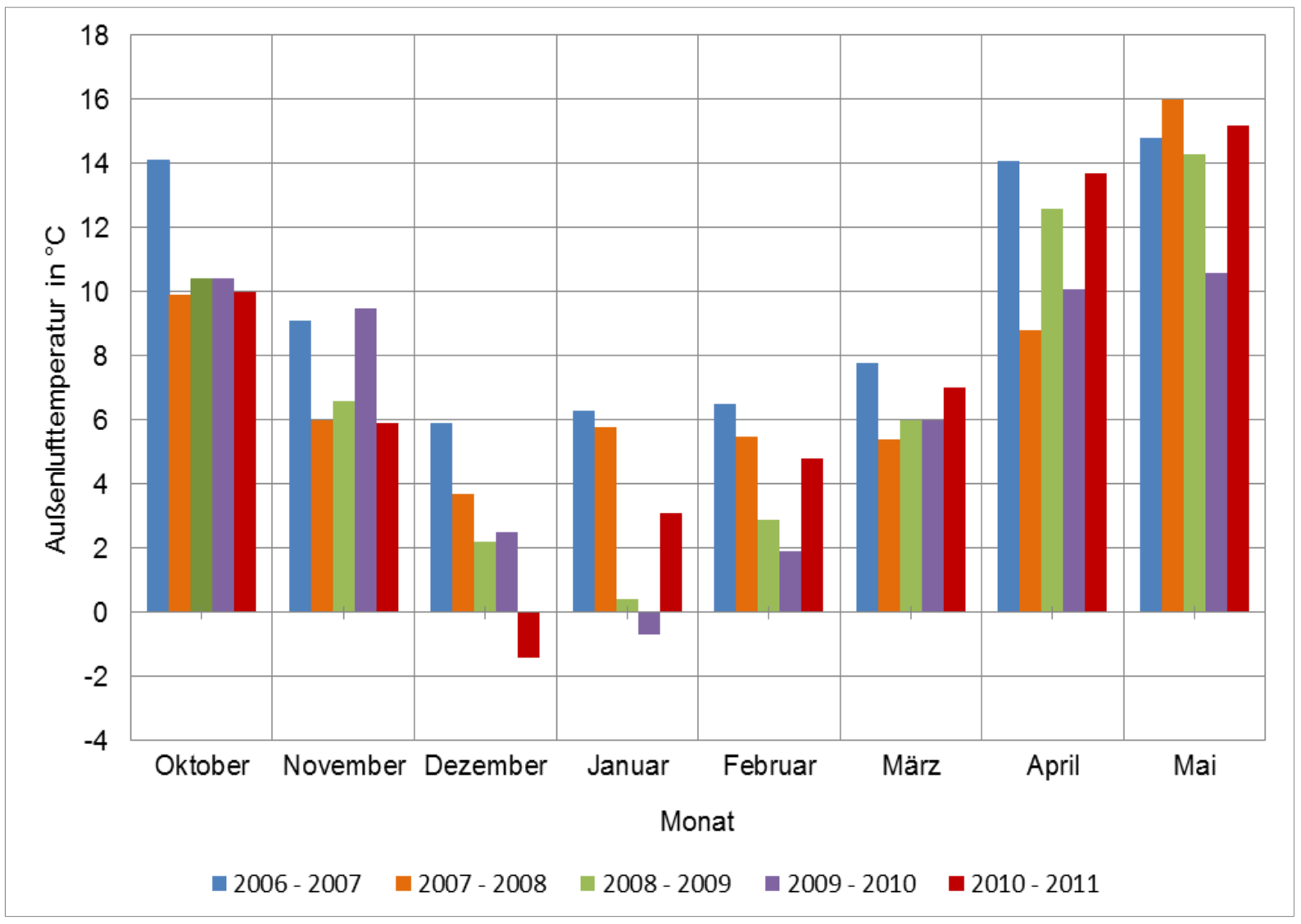

Abbildung 7.13: Außenlufttemperaturen in der Heizperiode für den Standort Aachen (Quelle DWD)

Die abgelesenen Energieverbräuche auf dem Nachtstromzähler sind in Tabelle 7.7 dargestellt. Es ist deutlich zu erkennen, dass eine Energieeinsparung von über 1400 kWh (> 15\%) für das hier untersuchte Objekt vorliegt. Diese Energieeinsparung ist als erstaunlich hoch anzusehen, insbesondere unter dem Aspekt des geringen Aufwandes.

Aufgrund der spezifischen Gegebenheiten des untersuchten Objekts ist eine Verallgemeinerung der Einsparung auf andere Objekte nicht möglich.

\begin{tabular}{|c|c|c|c|c|c|}
\hline Heizperiode: & $2006-2007$ & $2007-2008$ & $2008-2009$ & $2009-2010$ & $2010-2011$ \\
\hline $\begin{array}{c}\text { Jahresverbrauch in } \\
\text { kWh/a }\end{array}$ & 9260 & 9593 & $8230^{*}$ & 7621 & 7732 \\
\hline
\end{tabular}

*) der Jahresverbrauchswert ist für die ganze Heizperiode angegeben, wenngleich die Beschichtung erst innerhalb der Heizperiode, das heißt ab dem 11.12.2008, vorhanden war.

Tabelle 7.7: Energieverbräuche für ein Pilotobjekt 
Für das hier untersuchte Objekt kann von einer kurzen Amortisierung ausgegangen werden. Die Raumlufttemperatur im Versuchsobjekt wurde in der Zeit vom 16.11 .2009 bis zum 31.03.2010 aufgezeichnet, vergleiche Raum R9 in Kapitel 6.1. Abbildung 7.14 zeigt die gemessenen Raumlufttemperaturen. Es sind erstaunlich große Temperaturschwankungen festzustellen, welche durch die schlechte Regelung der Nachtspeicheröfen zu erklären sind.

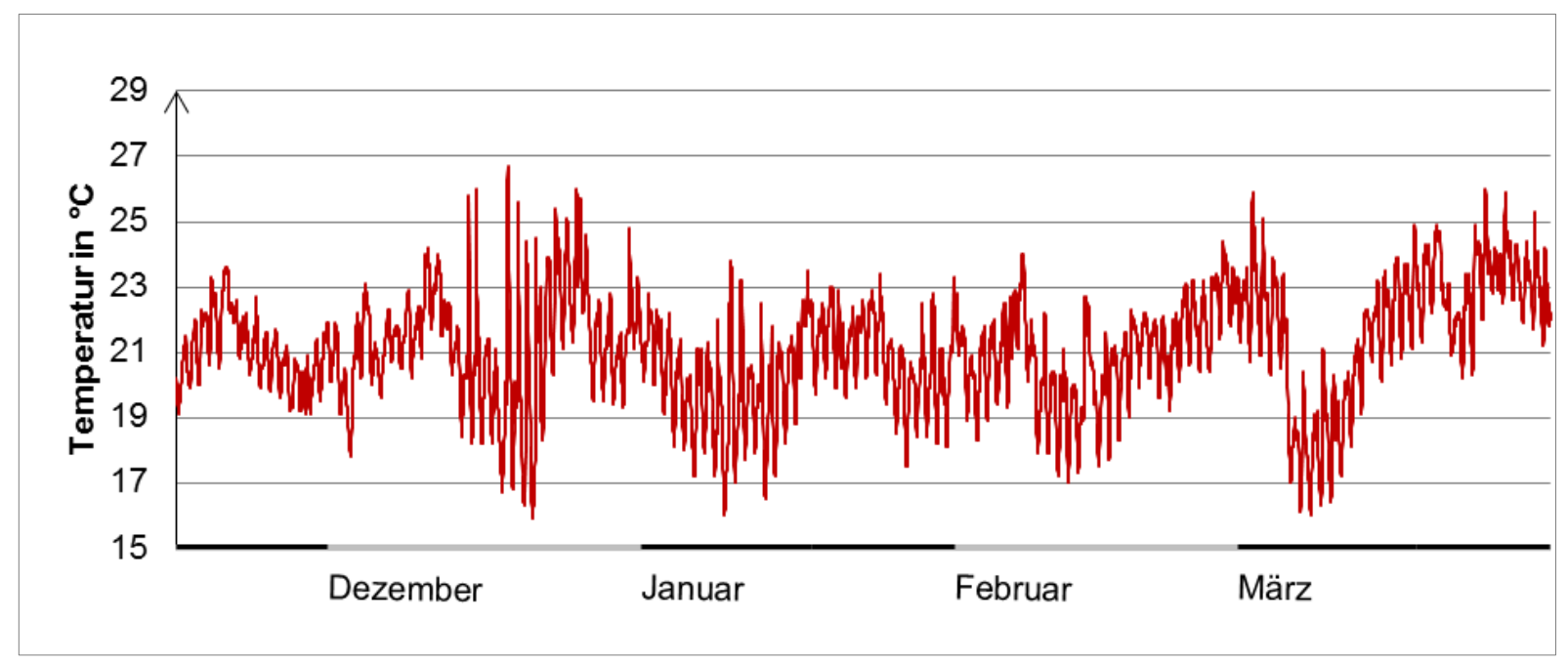

Abbildung 7.14: Raumlufttemperatur im Pilotobjekt nach dem Einbau

Ein besser regelbares Heizungssystem könnte hier einen ausgeglicheneren Temperaturverlauf herstellen, wodurch weitere Energieeinsparung denkbar wären. 


\subsection{1. $\quad$ Amortisationsbetrachtung für das Pilotobjekt}

In dem in Abschnitt 7.4 beschriebenen Pilotobjekt wurde nur eine Innenraumrenovierung durchgeführt, bei welcher eine Vliesschicht unter der Raufaserdeckschicht an den Wänden eingebaut wurde. Die Kosten für die Renovierung sind in Tabelle 7.14 dargestellt.

\begin{tabular}{|l|c|c|}
\hline Maßnahmen & Materialkosten & Lohnkosten \\
\hline Alte Tapete entfernen & & $4,0 € / \mathrm{m}^{2}$ \\
\hline Untergrund vorbereiten & & $4,0 € / \mathrm{m}^{2}$ \\
\hline Vliesschicht tapezieren & $6,0 € / \mathrm{m}^{2}$ & $4,0 € / \mathrm{m}^{2}$ \\
\hline Rauhfaser tapezieren & $0,5 € / \mathrm{m}^{2}$ & $2,5 € / \mathrm{m}^{2}$ \\
\hline $2 \times$ Anstreichen & $1,0 € / \mathrm{m}^{2}$ & $4,0 € / \mathrm{m}^{2}$ \\
\hline Gesamtkosten & $\mathbf{7 , 5} € / \mathrm{m}^{2}$ & $\mathbf{1 8 , 5} \in / \mathrm{m}^{2}$ \\
\hline Gesamtkosten ohne Vliesschicht & $1,5 € / \mathrm{m}^{2}$ & $14,5 € / \mathrm{m}^{2}$ \\
\hline Mehrkosten durch Vliesschicht & $\mathbf{6 , 0} € / \mathrm{m}^{2}$ & $\mathbf{4 , 0} € / \mathrm{m}^{2}$ \\
\hline
\end{tabular}

Tabelle 7.15: Material- und Lohnkosten für die Renovierungsarbeiten

Die Amortisationsberechnung wurde ohne Zinsgewinne und Zinsen für einen Kredit durchgeführt und die Kosten für die Kilowattstunde elektrischen Strom werden konstant mit 15 Cent pro Kilowattstunde angenommen. Der Strompreis ist aus der Stromabrechnung des Versorgers entnommen worden (hier liegt noch ein sehr günstiger Strompreis vor).

Zinsen sind nicht berücksichtigt, da die Inflationsrate (Verteuerungsrate) dem Zinssatz entgegensteht, so dass bei einer rechnerischen Betrachtung wie folgt gerechnet wurde:

reale Habenszinsen $=$ Habenszinsen - Inflationsrate - Bankgebüren

Leider zeigt die Berechnung unter dieser Annahme, dass das Geld im Regelfall bei der Bank zu Ungunsten des Kunden angelegt ist. Der Sollzinssatz wurde nicht angesetzt, weil davon auszugehen ist, dass für die errechneten Maßnahmen in der Regel kein Kredit notwendig ist.

Im betrachteten Amortisierungszeitraum werden für Renovierungsaufwände wie Neuanstriche und andere Kosten hinzukommen. Da diese Renovierungsarbeiten durch die Vliesschicht nicht beeinflusst werden, sind diese Kosten jedoch unabhängig von der energieeinsparenden Maßnahme zu sehen und werden daher nicht miteinkalkuliert.

Bei den gegebenen Randbedingungen ist eine Amortisationszeit von fünf Jahren für die Mehrkosten und von 13 Jahren für die komplette Renovierungsmaßnahme, ohne Abzug der sowieso üblichen Renovierungskosten, zu erwarten, vergleiche Abbildung 7.16. 


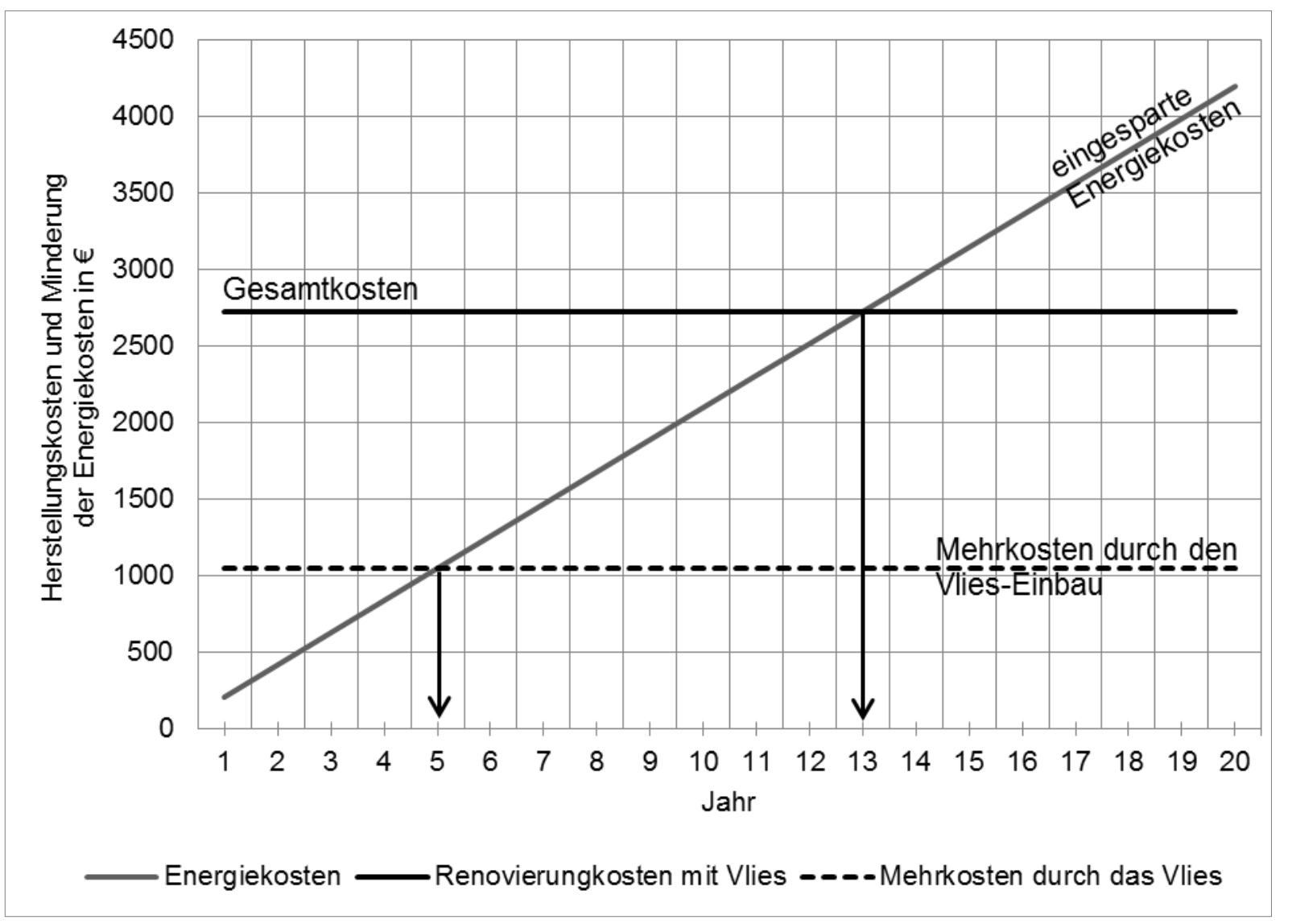

Abbildung 7.16: Amortisationszeiten für das hier untersuchte Pilotobjekt

Unter der Annahme, dass die Energieeinheitskosten steigen, ist eine schnellere Amortisation zu erwarten. Es ist hier die ungünstigste Amortisationssituation dargestellt, um eine gute Planungs- und Finanzierungssicherheit zu gewährleisten.

Der Vorteil der besseren Behaglichkeit lässt sich kostenmäßig nicht darstellen.

Die gemessenen Raumtemperaturen haben gezeigt, dass die sehr ungenaue Temperaturregelung aufgrund der Nachtspeicheröfen oft zu einer unnötigen Überhitzung der Räume führt.

Für das betrachtete Objekt sollte in einem nächsten Schritt die Heizungssteuerung beziehungsweise das Heizungssystem verbessert oder ausgetauscht werden. Durch ein neues Heizungssystem ist zu erwarten, dass weitere Energie eingespart werden kann und somit die Investitionskosten für ein neues Heizungssystem in einem angemessenen Zeitraum amortisierbar sind. 


\subsection{Ergebnisdiskussion}

Die Ergebnisse aus den Laborversuchen zeigen, dass sehr dünne Dämmschichten ohne zusätzliche Verkleidungen (Gipskarton oder dergleichen) an der Bauteiloberfläche das thermische Verhalten, das heißt die Oberflächentemperatur sowie die Raumlufttemperatur, günstig beeinflussen.

Durch dünne Dämmschichten auf den Wandoberflächen im Massivbau können schnellere Raumanheizphasen realisiert werden. Dies führt beim üblichen intermittierenden Heizbetrieb zu beachtlichen Energieeinsparungen, vergleiche Kapitel 7.5.

Dünne, raumseitig aufgebrachte Dämmschichten von bis zu $4 \mathrm{~mm}$ an den Innen- und AuBenwänden verkürzen die Anheizphase erheblich und dennoch ist der Wärmespeicher in Form einer massiven Wand sehr gut wirksam und hinsichtlich der Energieeinsparung und Behaglichkeit sehr effizient wirksam. Es wird deutlich, dass auch bei einer Heizungsnachtabschaltung die massiven Bauteile (Wände) nicht so stark auskühlen, wie dies ohne eine dünne Dämmschicht der Fall ist. Stattdessen bleibt die Raumluft durch die gespeicherte Wärme in den Bauteilen auch noch einige Zeit nach der Heizungsabschaltung in einem günstigen operativen Temperaturniveau.

Die Ergebnisse aus dem bewohnten Pilotobjekt zeigen, dass über den Zeitraum von 2008 bis 2011 mit der Vliesbeschichtung nennenswerte Energieeinsparungen erzielt werden konnten. Durch den relativ minimalen baulichen Eingriff ist zudem eine rasche Amortisation der Zusatzkosten möglich.

Der Einbau der beschriebenen dünnen Dämmschicht bietet sich insbesondere für den Gebäudebestand an, wo Lösungen zur Reduzierung der Heizenergie gesucht werden, welche mit kleinem baulichen Aufwand und mit geringen Kosten erreicht werden können. 


\section{Rechnerische Untersuchung}

\subsection{Vorgehensweise}

Die rechnerischen Untersuchungen ermöglichen mit wenig Aufwand den thermischen Einfluss der dünnen Dämmschicht auf ein Wandsystem zu überprüfen. Hierdurch können verschiedene Wandsysteme sowie unterschiedliche Temperaturrandbedingungen analysiert werden.

In Kapitel 8.2 werden die Ergebnisse aus dem Laborprüfstand mit der Berechnung validiert. Für die Berechnungen wurde die Software „HeatTransmission“ der Firma SmartCAE verwendet, welche mit der Mathematica-Umgebung läuft.

Die Software „HeatTransmission“ erlaubt es, komfortabel den instationären Wärmetransport durch mehrschichtige ebene Konstruktionen mit unterschiedlichen und auch sehr dünnen Schichten zu berechnen.

Die oben genannte Software ist sehr flexibel, so dass Temperatur-Zeitverläufe auch über große Zeiträume leicht zu berechnen sind. Herr Stefan Braun von der Firma SmartCAE ergänzte für diese Arbeit den Funktionsumfang der Software, wodurch nun beispielsweise periodische Temperaturverläufe automatisch generiert werden können.

\subsection{Validierung der Berechnungen mit der Messergebnissen}

Die rechnerischen Ermittlungen wurden unter den Randbedingungen, welche sich im LaborPrüfstand ergaben, verglichen, um zu sehen, ob das verwendete numerische Rechenmodell zu gleichen oder ähnlichen Ergebnissen führt.

Für die Berechnung sind folgende Randbedingungen an den Wandoberflächen angenommen worden:

$\begin{array}{ll}\text { Wärmeübergang durch Konvektion: } & 3 \frac{\mathrm{W}}{\mathrm{m} \cdot \mathrm{K}} \\ \text { Emissionsgrad: } & 0,93\end{array}$

Die Raumlufttemperaturen wurden für die Berechnungen aus dem jeweiligen Versuch übernommen.

Es zeigt sich, dass die gerechneten Bauteiloberflächentemperaturen mit den gemessenen Temperaturen recht gut übereinstimmen, vergleiche Abbildungen 8.1 - 8.2. 


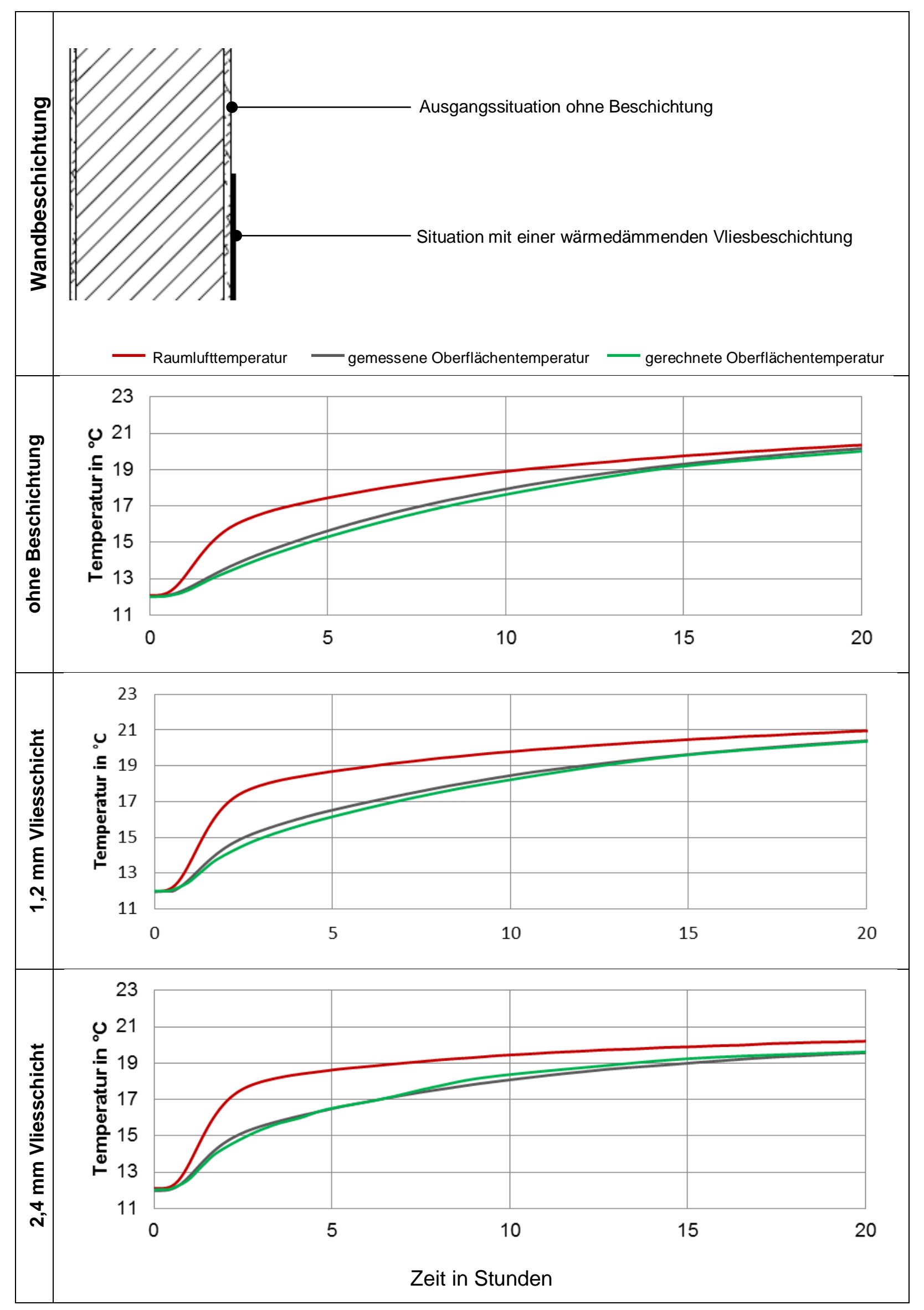

Abbildung 8.1: Vergleich der gemessenen und gerechneten Wandoberflächentemperaturen 


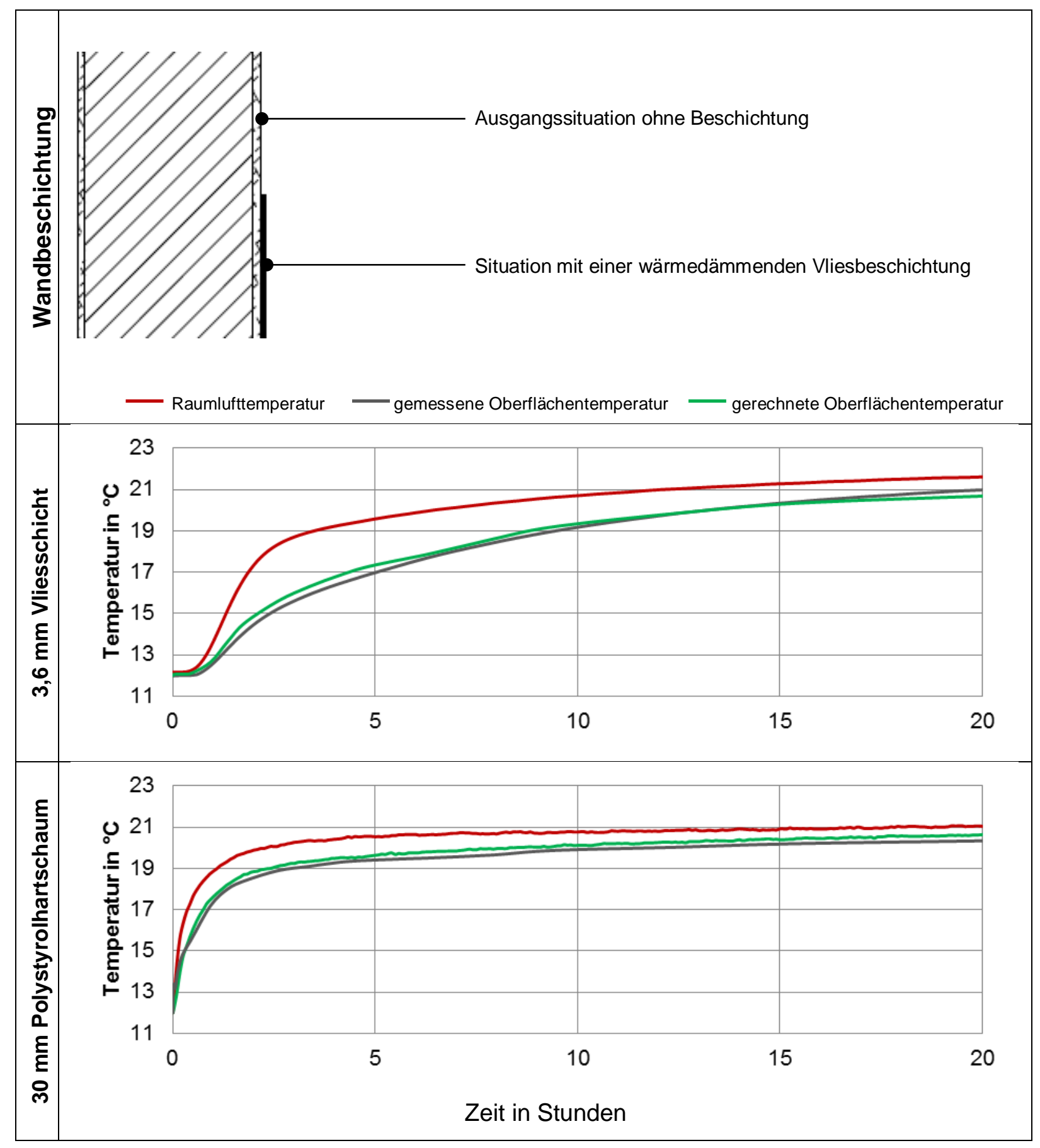

Abbildung 8.2: Vergleich der gemessenen und gerechneten Wandoberflächentemperaturen 


\subsection{Thermisches Verhalten von Innenwandkonstruktionen}

Für fünf typische Innenwandkonstruktionen ohne und mit einer Vliesschicht sind die Wandoberflächentemperaturen berechnet und analysiert worden.

Dabei wurden leichte Trennwände, als auch mittelschwere und schwere Mauerwerks- beziehungsweise Betonwände untersucht.

Folgende Innenwandkonstruktionen wurden jeweils mit einer einseitigen Vliesbeschichtung rechnerisch untersucht:

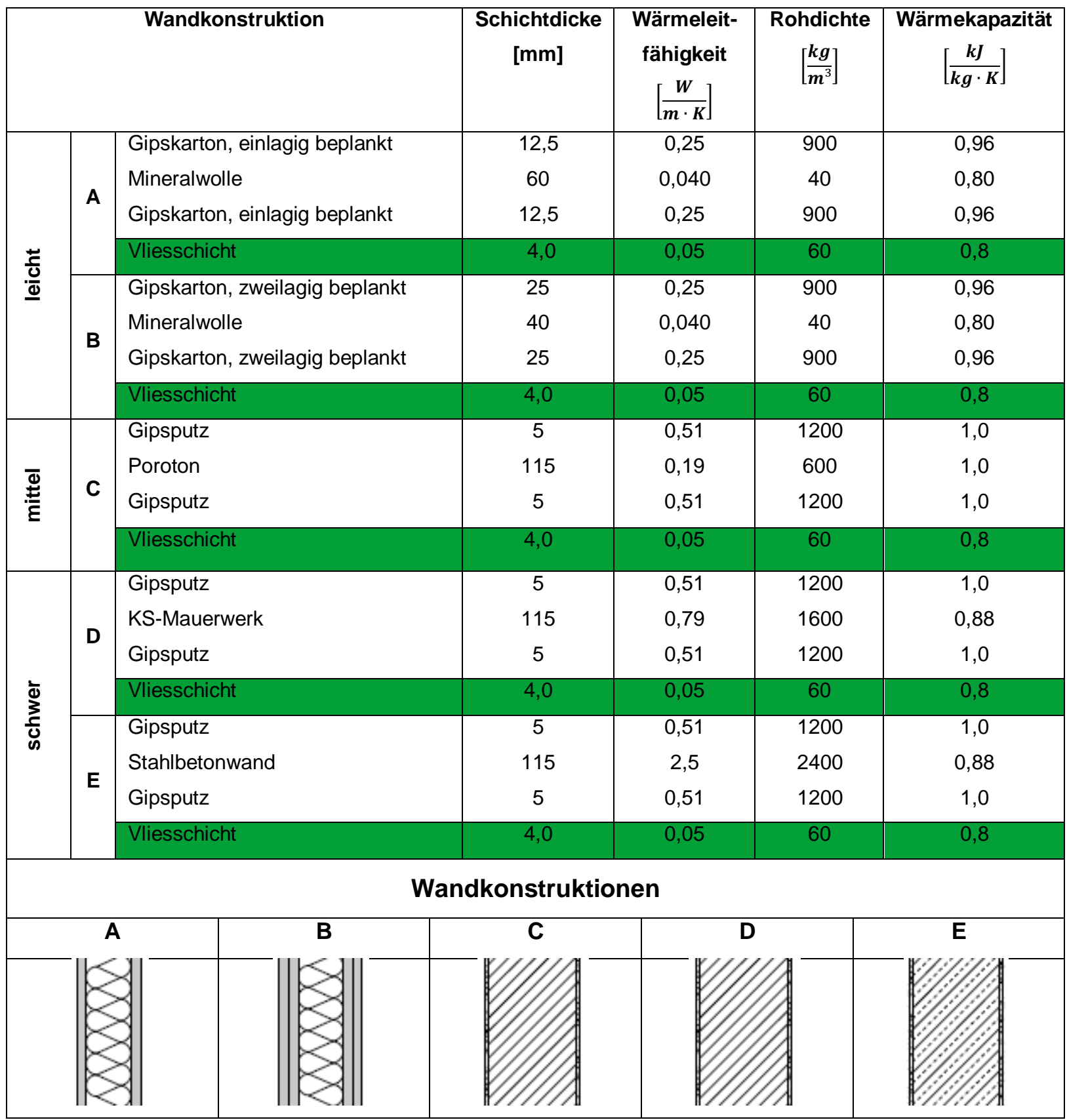

Tabelle 8.1: Wandkonstruktionen, an welchen thermische Berechnungen vorgenommen wurden 
Die Berechnungen wurden bei folgenden Randbedingungen an den Wandoberfläche durchgeführt:

Wärmeübergang durch Konvektion: $\quad 3 \frac{\mathrm{W}}{\mathrm{m} \cdot \mathrm{K}}$

Emissionsgrad: $\quad 0,93$

\subsubsection{Innenwandkonstruktionen bei instationärem Heizbetrieb}

Die beschriebenen Wandkonstruktionen nach Tabelle 8.1 sind bei einem intermittierenden Heizbetrieb mit der Software „HeatTransmission“ berechnet worden.

Für die Berechnung wird der Raumlufttemperaturabfall und die Wiederaufheizzeit in Anlehnung an DIN EN 12831:2003 (D) angenommen. Der untersuchte Raumlufttemperaturabfall wird mit 2 Kelvin, 3 Kelvin und 4 Kelvin festgelegt, um verschiedene thermische Verhältnisse, abhängig vom Außenklima und dem Wärmedämmniveau eines Raumes, zu simulieren.

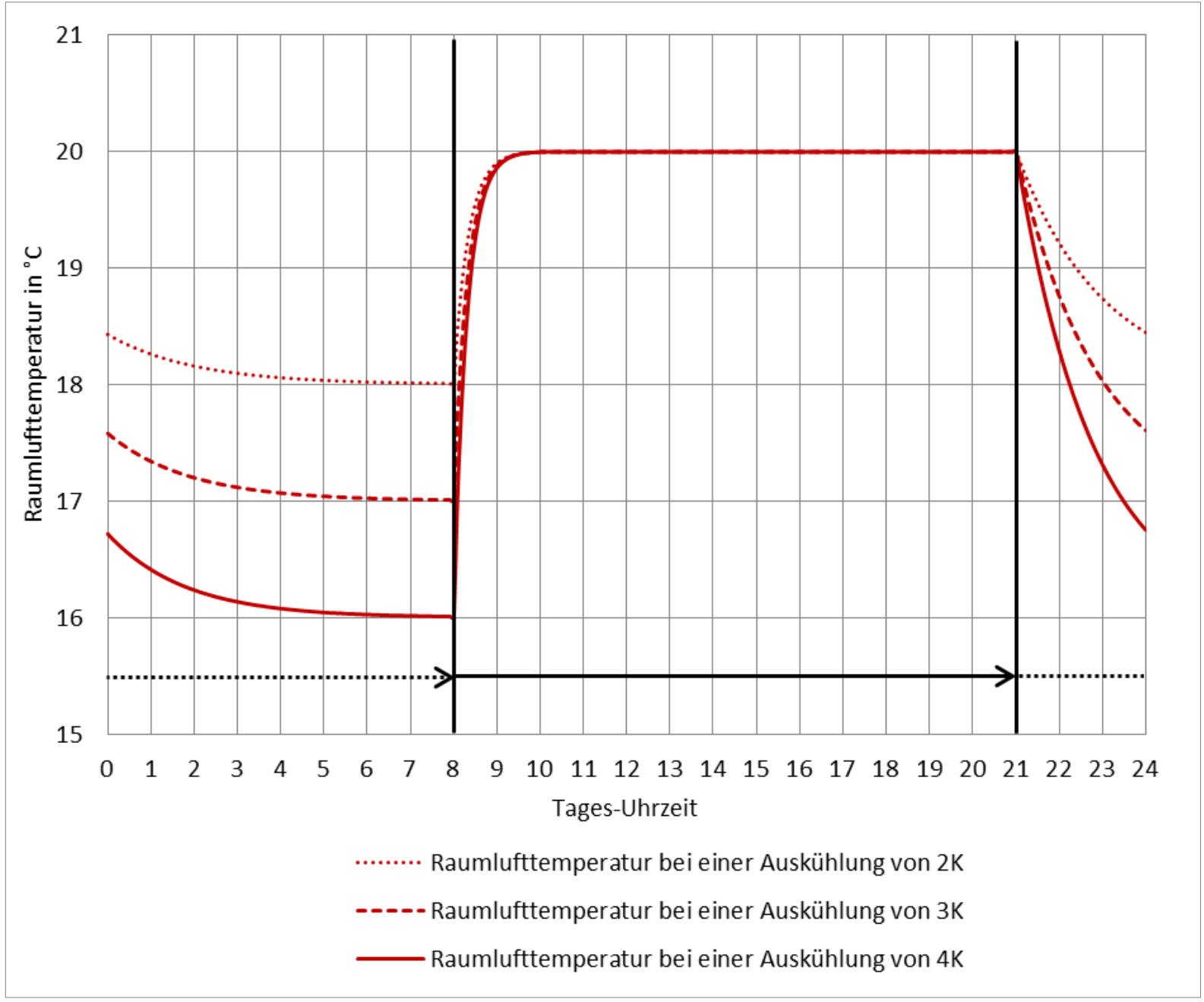

Abbildung 8.3: Angenommene Raumlufttemperaturverläufe bei einer Raumlufttemperatur-Nachtabsenkung von $2 \mathrm{~K}$, $3 \mathrm{~K}$ und $4 \mathrm{~K}$ 
Bei den Berechnungen wurde für sämtliche berechnete Oberflächentemperaturen derselbe Temperaturverlauf der Raumluft zu Grunde gelegt. In der Praxis steht die Raumlufttemperatur in einer gewissen Abhängigkeit der Oberflächentemperatur, so dass bei einer massiven Bauweise die Raumlufttemperatur nicht so schnell abfällt und der Raumlufttemperaturanstieg bei einer Leichtkonstruktion vergleichsweise schneller erfolgt.

Zum Vergleich der Konstruktionen wurde für die Berechnungen eine „erzwungene Raumlufttemperatur“, unabhängig von den Speichermassen, zu Grunde gelegt.

In der folgenden Abbildung 8.2 sind die Temperaturentwicklungen an den Wandoberflächen, bei den verschiedenen Wandkonstruktionen A bis $\mathrm{E}$ nach Tabelle 8.1 dargestellt. 


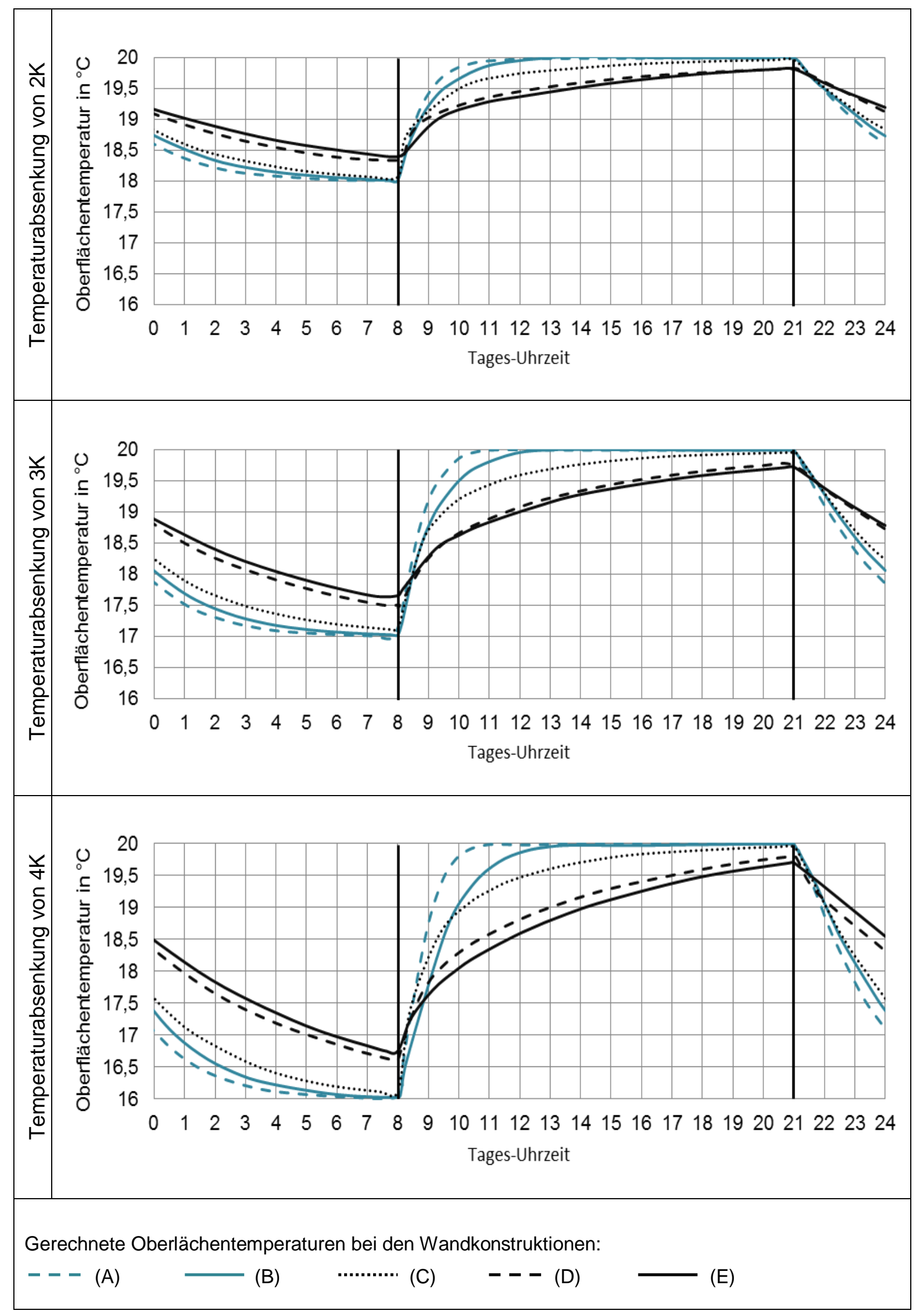

Abbildung 8.2: Wandoberflächentemperaturverläufe für die Wandkonstruktionen nach Tabelle 8.1 
Um die Wandkonstruktionen mit und ohne Vliesschicht vergleichen zu können, werden für die Wandkonstruktionen aus Tabelle 8.1 die Oberflächentemperaturen auf der Vliesschicht und der unbeschichteten, normalen Wand berechnet.

Für die Kalkulation werden die Wandkonstruktionen mit unterschiedlichen Raumlufttemperaturverläufen analysiert, das heißt bei Raumluft-Temperaturabfällen von 2 Kelvin, 3 Kelvin und 4 Kelvin in der Nachtzeit, vergleiche Abbildung 8.2.

Die Raumluftemperatur wurde als „erzwungene Temperaturentwicklung“ zu Grunde gelegt. Der Einfluss der Speichermassen auf die Raumluft wurde daher nicht berücksichtigt. In der Praxis ist dieser Einfluss vorhanden, wie dies aus den Kapiteln 7.2 und 7.4, welche Messergebnisse aus Objekten und einem Prüfstand im Labor zeigen, hervorgeht.

In den Abbildungen 8.3 bis 8.8 werden die Oberflächentemperaturen sowie die Temperaturdifferenzen zwischen der Situation ohne und mit einer Vliesschicht dargestellt. Hierdurch ist für die jeweilige Wandkonstruktion eine einfache Gegenüberstellung der thermischen Auswirkungen möglich. 
A) Leichte Wandkonstruktion, einfach beplankt

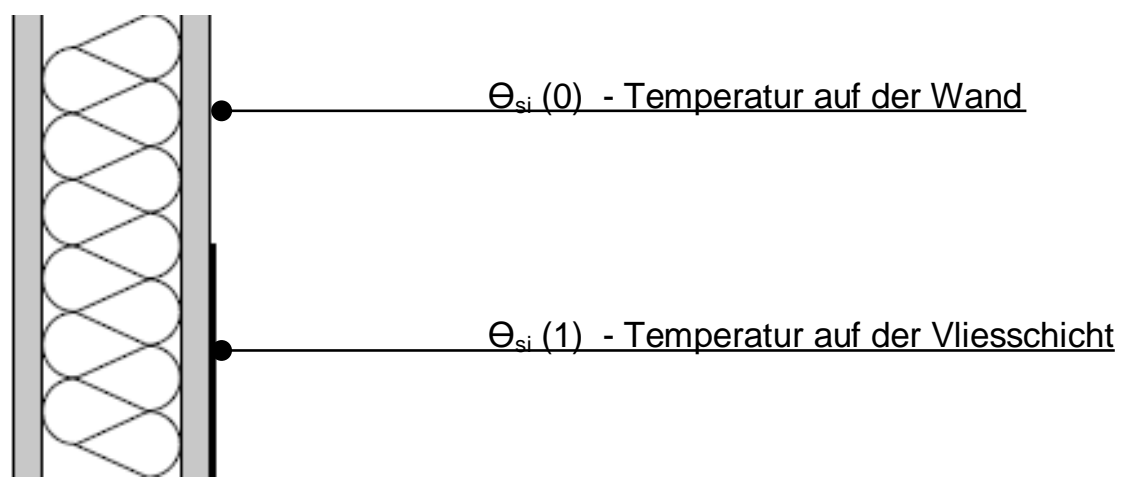

Bauteilschichten: Gipskarton, Mineralwolle, Gipskarton, (Vliesschicht)

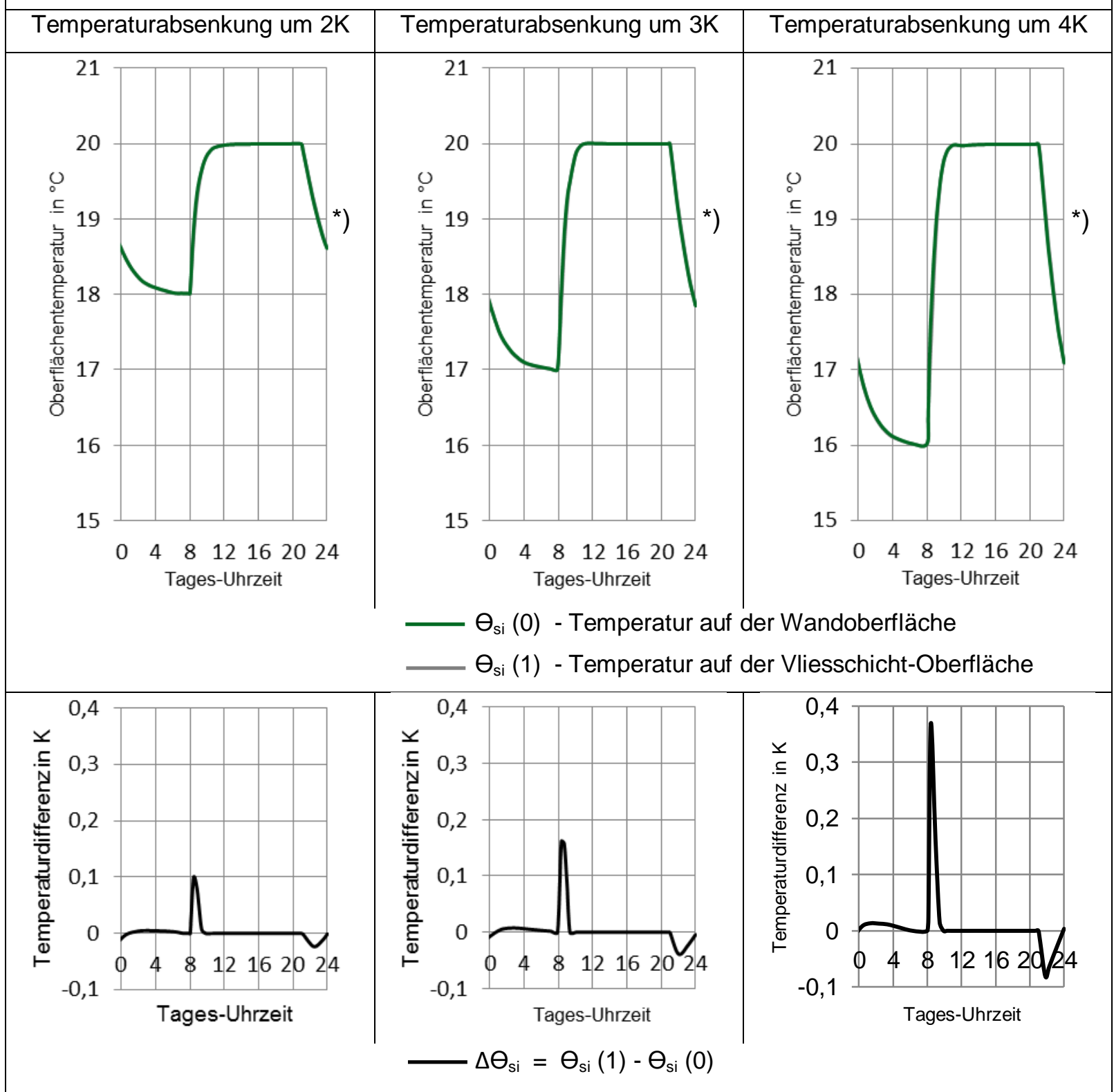

*) Der Unterschied der Temperaturkurven kann aufgrund der Diagrammauflösung hier nicht dargestellt werden 
B) Leichte Wandkonstruktion, zweifach beplankt

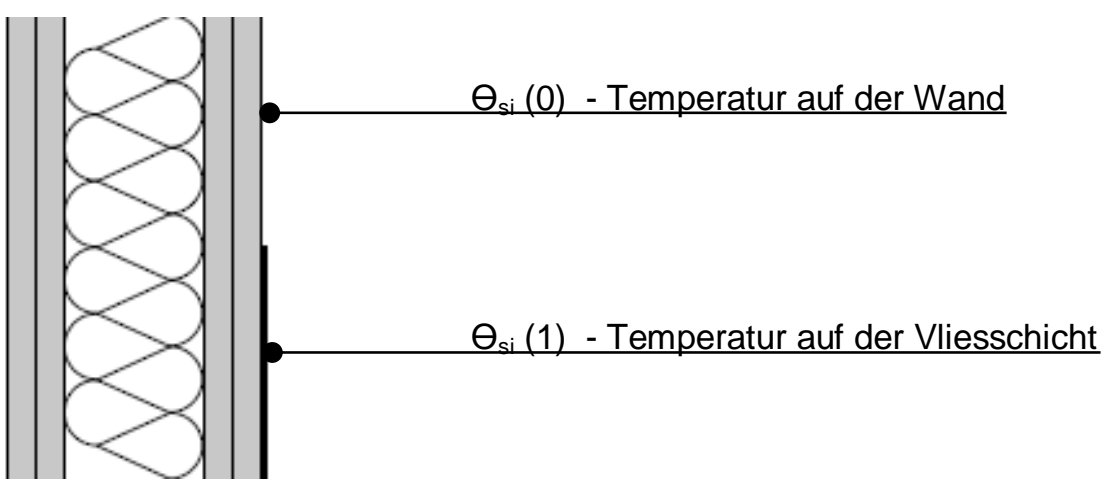

Bauteilschichten: 2 x Gipskarton, Mineralwolle, 2 x Gipskarton, (Vliesschicht)

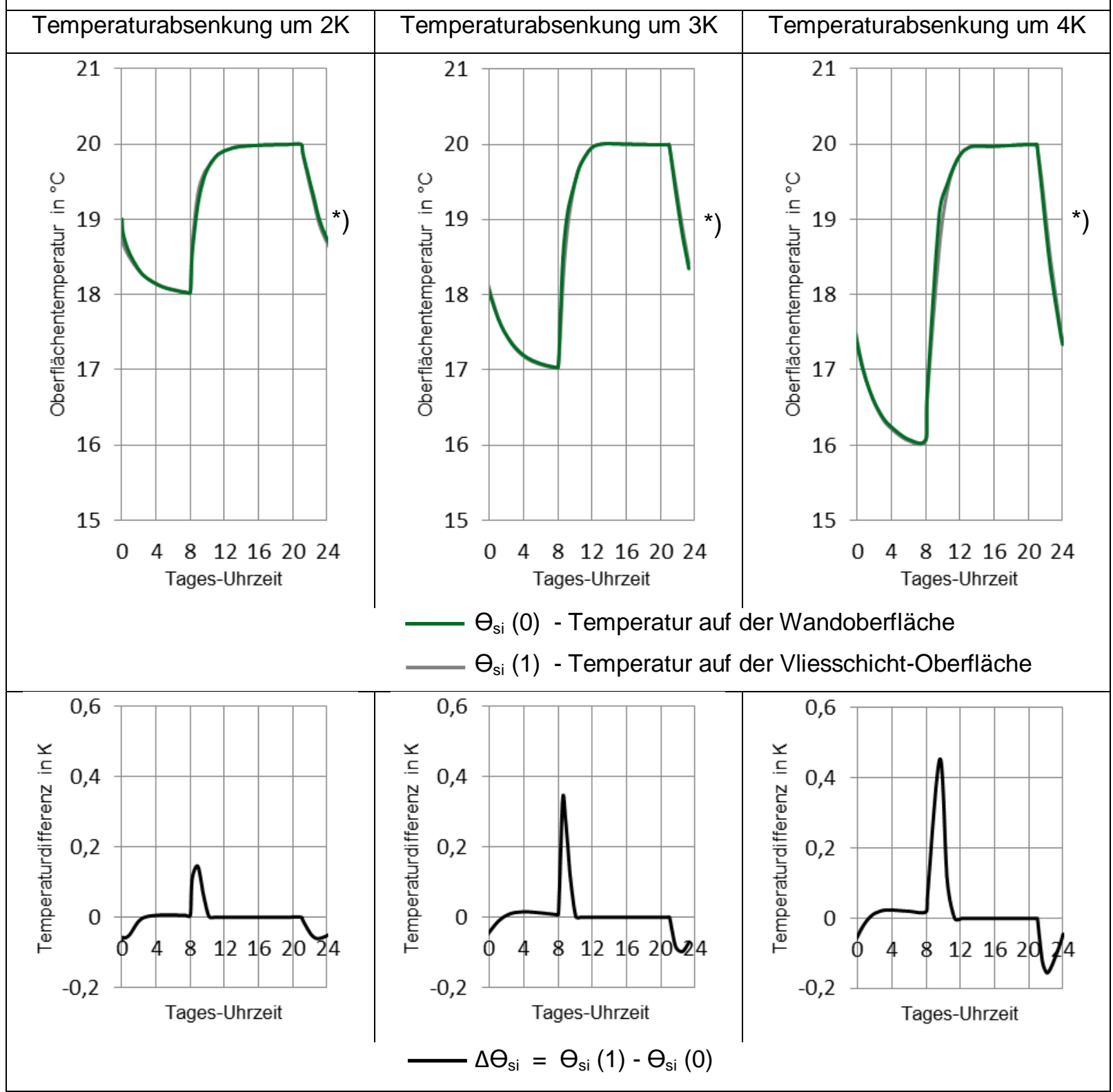

*) Der Unterschied der Temperaturkurven kann aufgrund der Diagrammauflösung hier nicht dargestellt werden 
C) Mittelschwere Wandkonstruktion

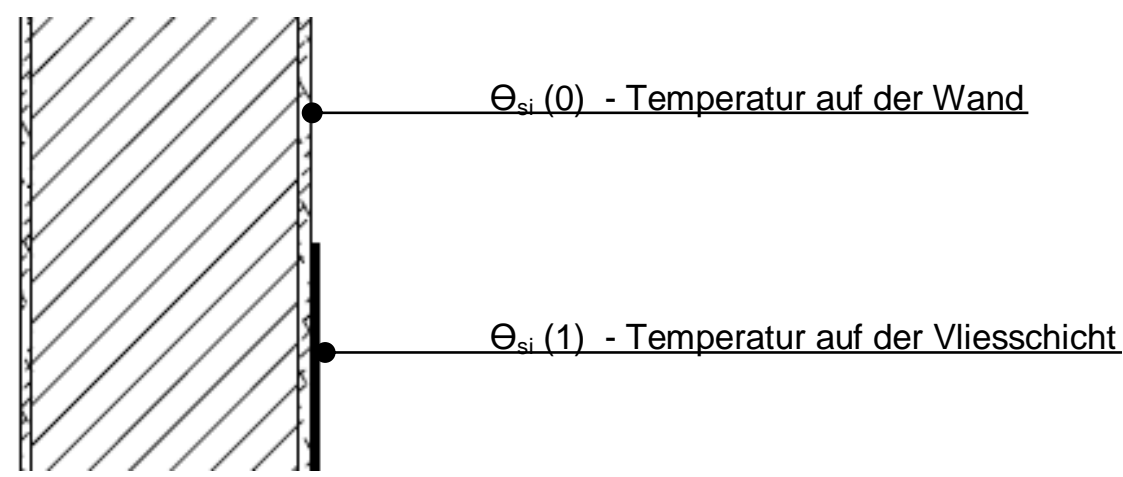

Bauteilschichten: Gipsputz, Poroton, Gipsputz, (Vliesschicht)

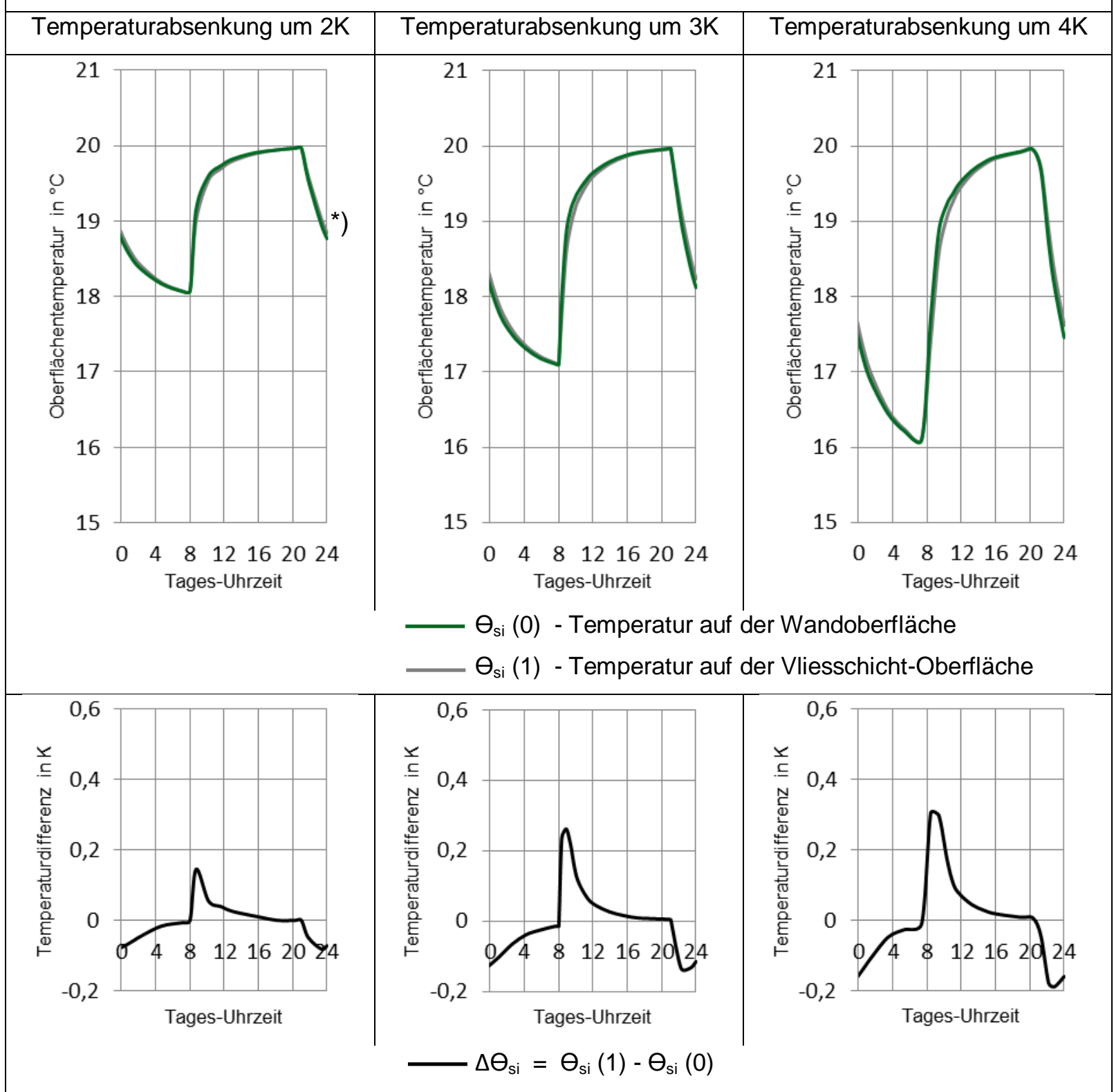

*) Der Unterschied der Temperaturkurven kann aufgrund der Diagrammauflösung hier nicht dargestellt werden 


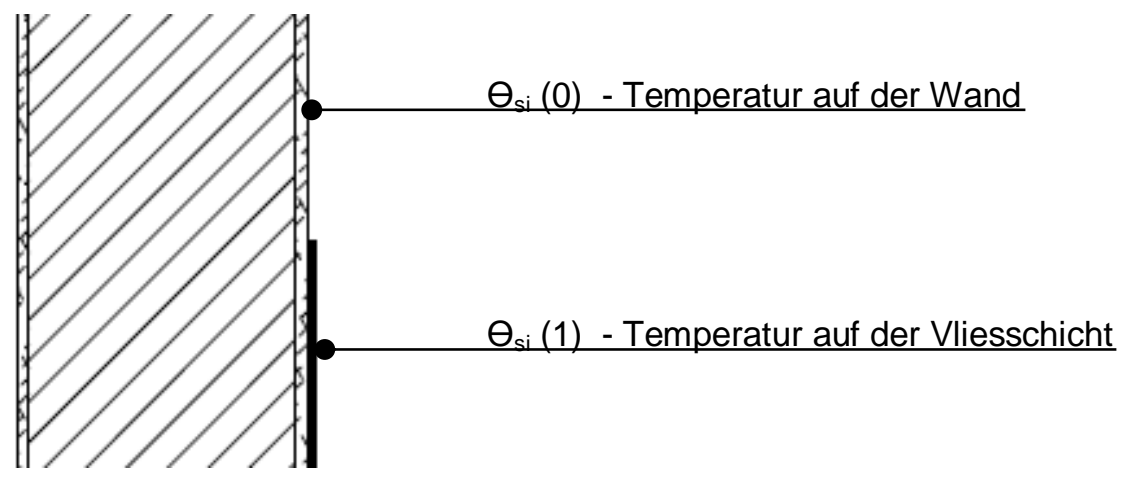

Bauteilschichten: Gipsputz, Kalksandstein, Gipsputz, (Vliesschicht)

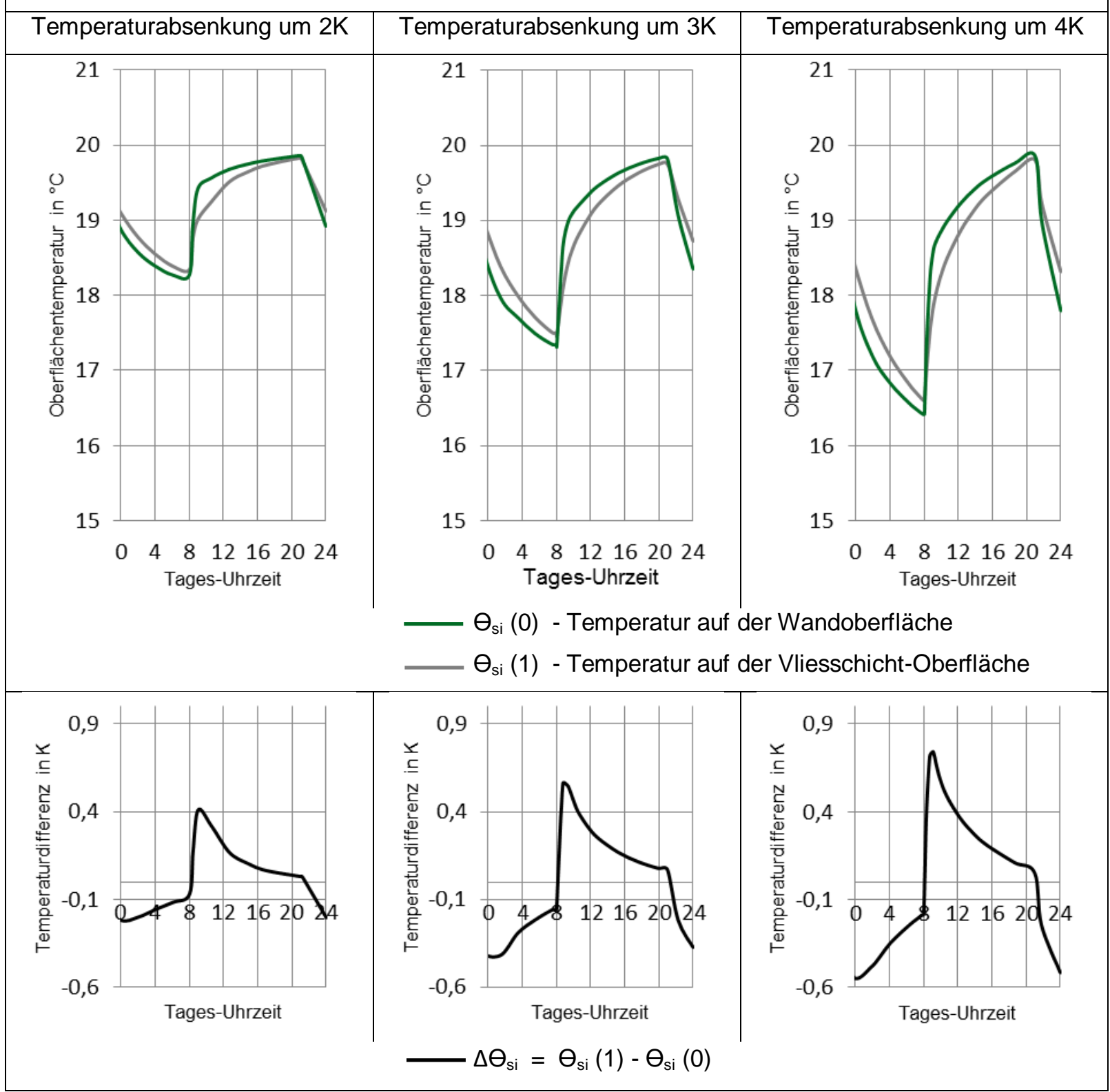

Abbildung 8.6: Wandoberflächentemperaturverläufe für Wandkonstruktion $D$ nach Tabelle 8.1 


\section{E) Schwere Wandkonstruktion}

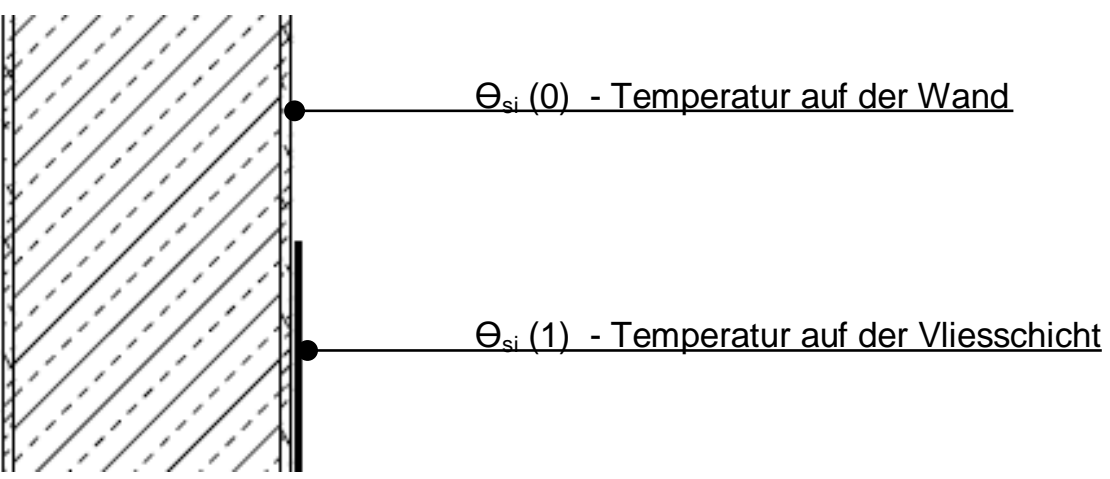

Bauteilschichten: Gipsputz, Stahlbeton, Gipsputz, (Vliesschicht)

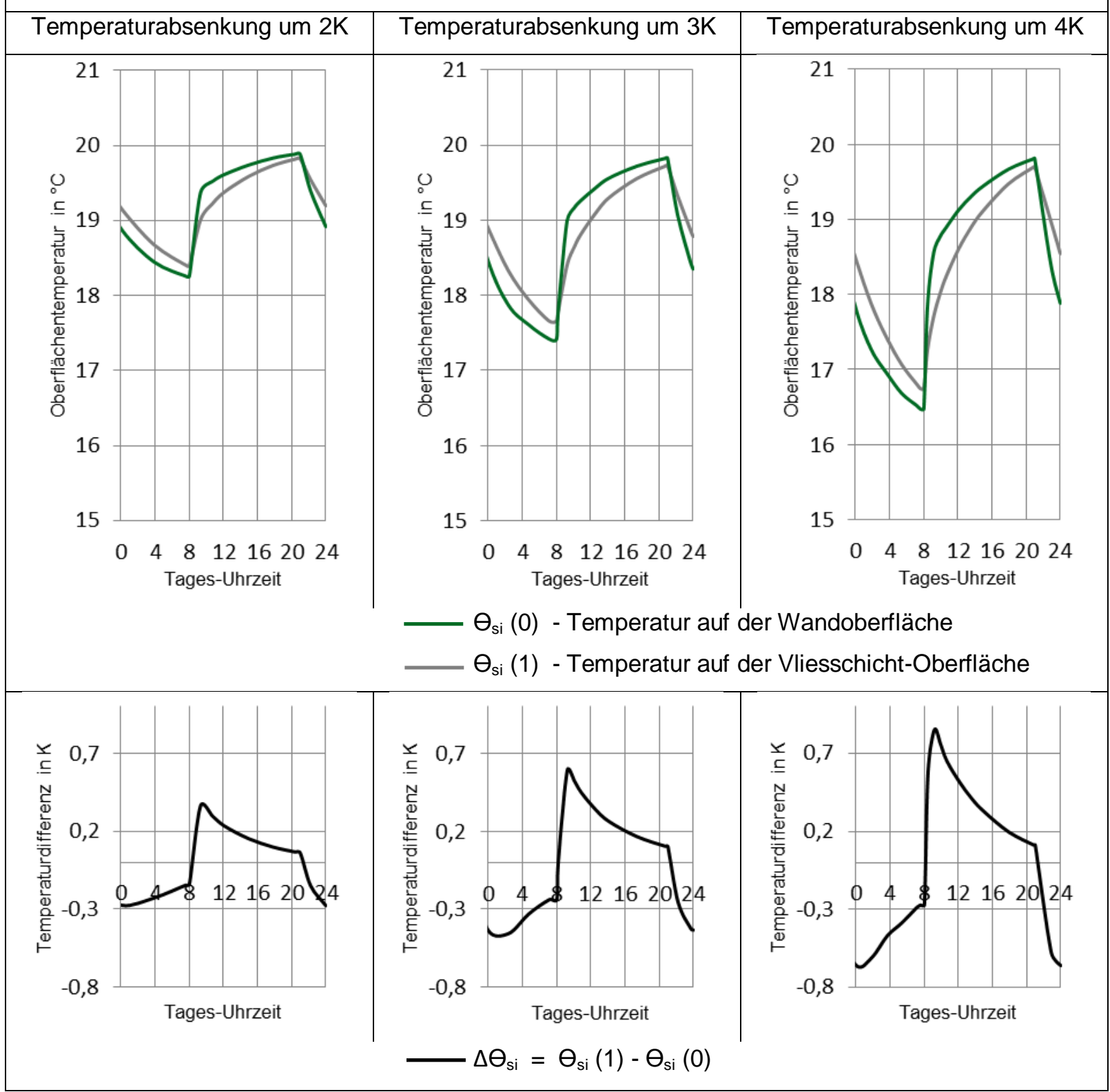

Abbildung 8.7: Wandoberflächentemperaturverläufe für Wandkonstruktion E nach Tabelle 8.1 


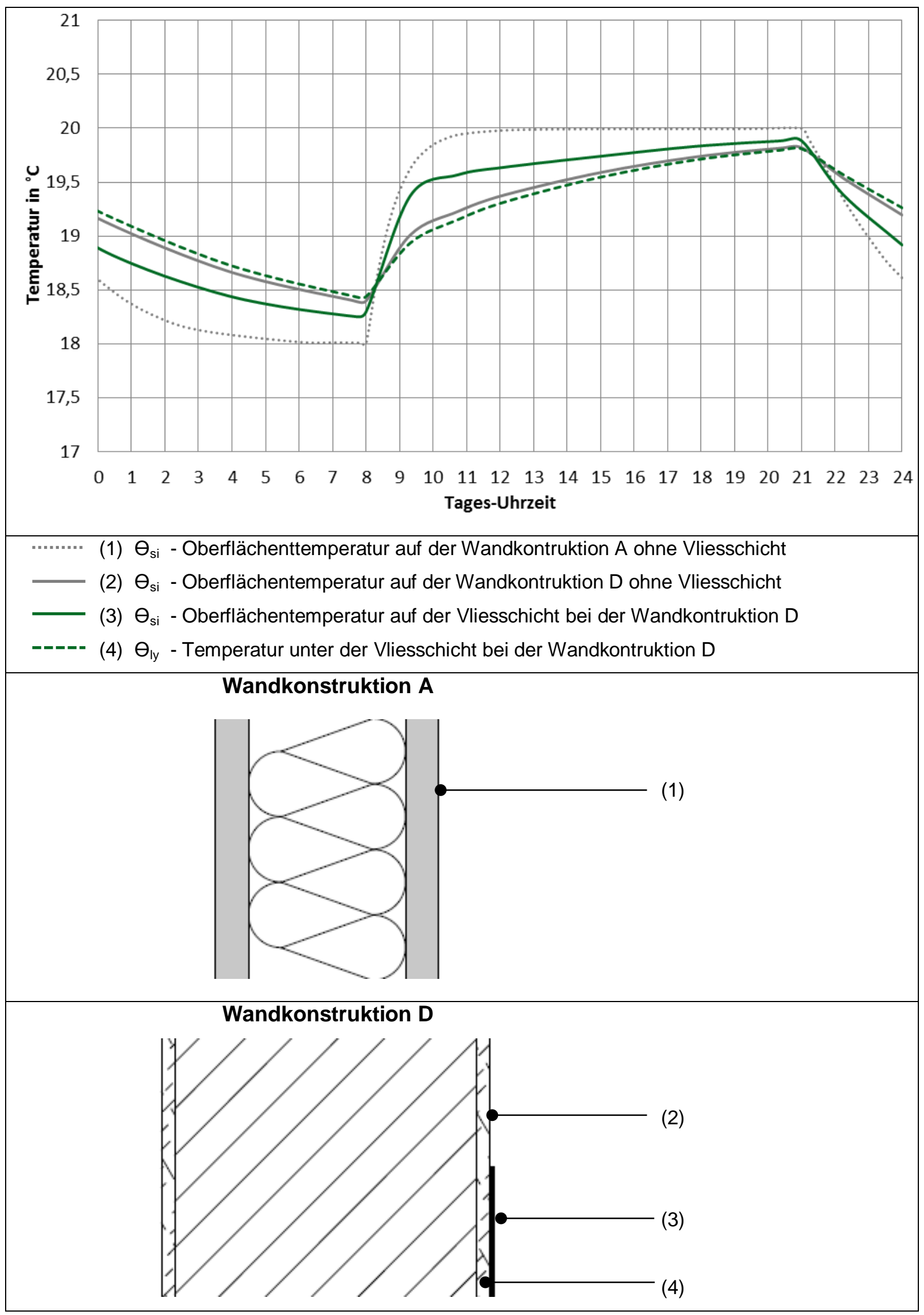

Abbildung 8.8: Wandoberflächentemperaturverlaufsvergleich für die Wandkonstruktionen A und D nach Tabelle 8.1 
Die Vergleiche der unterschiedlichen Wandkonstruktionen und der unterschiedlichen Raumlufttemperaturabsenkungen zeigen den Vorteil einer Vliesschicht bei einer intermittierenden Beheizung.

In der Praxis bedeutet dies, dass Gebäude oder auch nur einzelne Räume, welche seltener beheizt werden oder die aufgrund eines geringen Wärmeschutzes einer starken Auskühlung in der Nacht unterliegen, von den Vliesschichten in starkem Maße profitieren. Wände in massiver Bauweise, so wie diese im Gebäudebestand in der Regel vorzufinden sind, erzielen mit einer Vliesschicht sehr günstige thermische Verhältnisse bei einer intermittierenden Beheizung. Somit werden durch die Vliesschicht eine Verbesserung der Behaglichkeit in der Beheizungszeit sowie beachtliche Energieeinsparungen möglich. 


\subsection{Thermisches Verhalten von Außenwandkonstruktionen}

Für vier typische Außenwandkonstruktionen wird im Folgenden der Einfluss durch eine Vliesschicht berechnet, vergleiche Tabelle 8.10. Es wurden Außenwandkonstruktionen mit und ohne Wärmedämmung betrachtet. Hierdurch lassen sich unterschiedliche Dämmniveaus und der Einfluss der Vliessicht auf die Wand vergleichen.

Das Außenklima wurde für die Berechnungen konstant mit $0^{\circ} \mathrm{C}$ angenommen, wobei das Innenklima intermittierend mit einer Raumlufttemperatur-Nachtabsenkung von 2 Kelvin angenommen wurde, vergleiche Abbildung 8.9. In der Praxis sind Temperaturschwankungen beim Außenklima sowie solare Einstrahlungen durch die Verglasung vorhanden. Hierdurch wird die thermische Wirkung der Vliesschicht nochmals deutlich begünstigt, vergleiche die Versuchsergebnisse aus Kapitel 7.3.

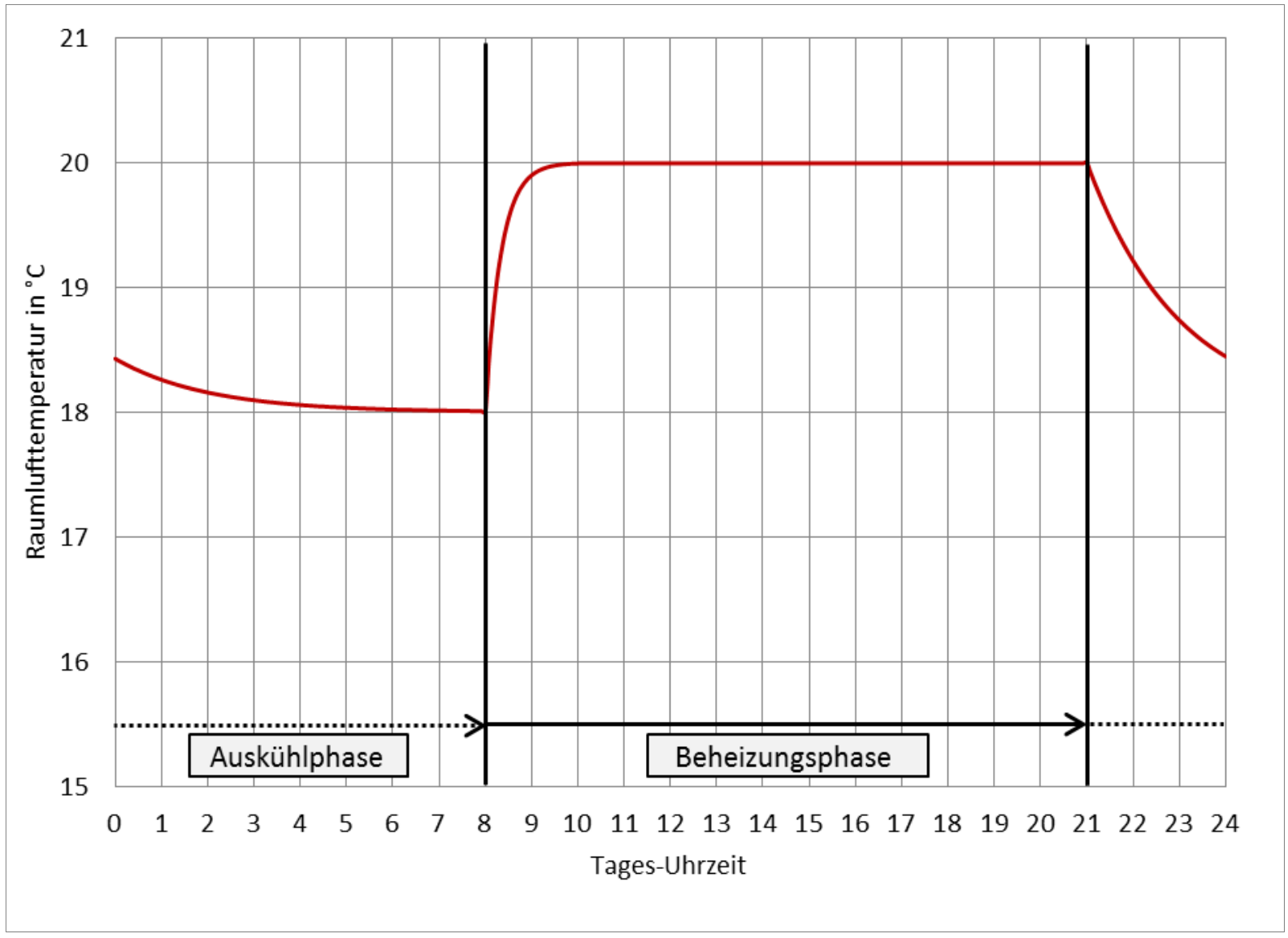

——Raumlufttemperatur bei einer Auskühlung von $2 \mathrm{~K}$

Abbildung 8.9: Angenommener Raumlufttemperaturverlauf bei einer Raumlufttemperatur-Nachtabsenkung von 2 Kelvin 
Folgende Wandkonstruktionen wurden betrachtet:

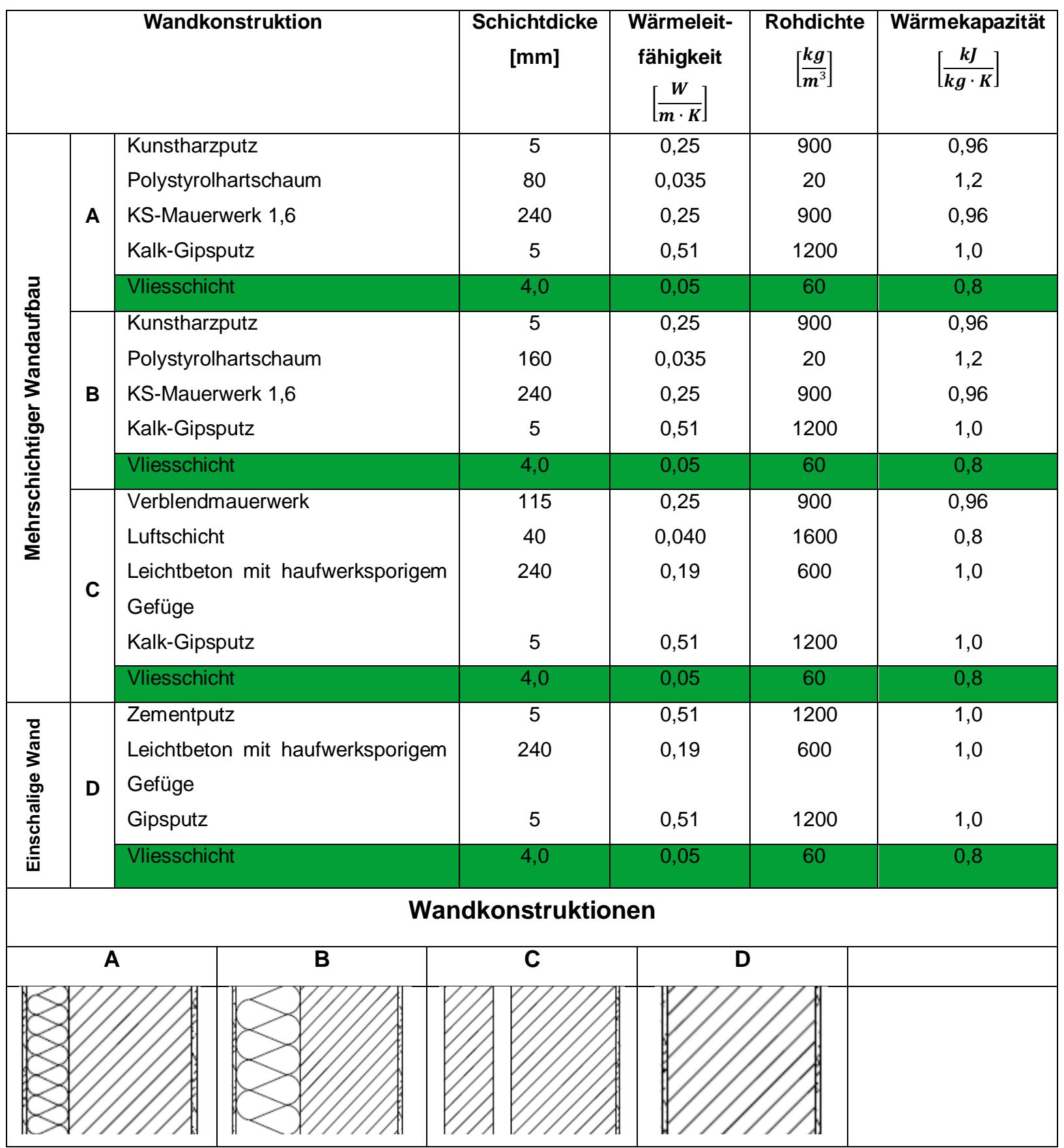

Abbildung 8.10: Außenwandkonstruktionen, an welchen thermische Berechnungen vorgenommen wurden 
A) Kalksandstein-Mauerwerk mit 80 mm Außenwanddämmung

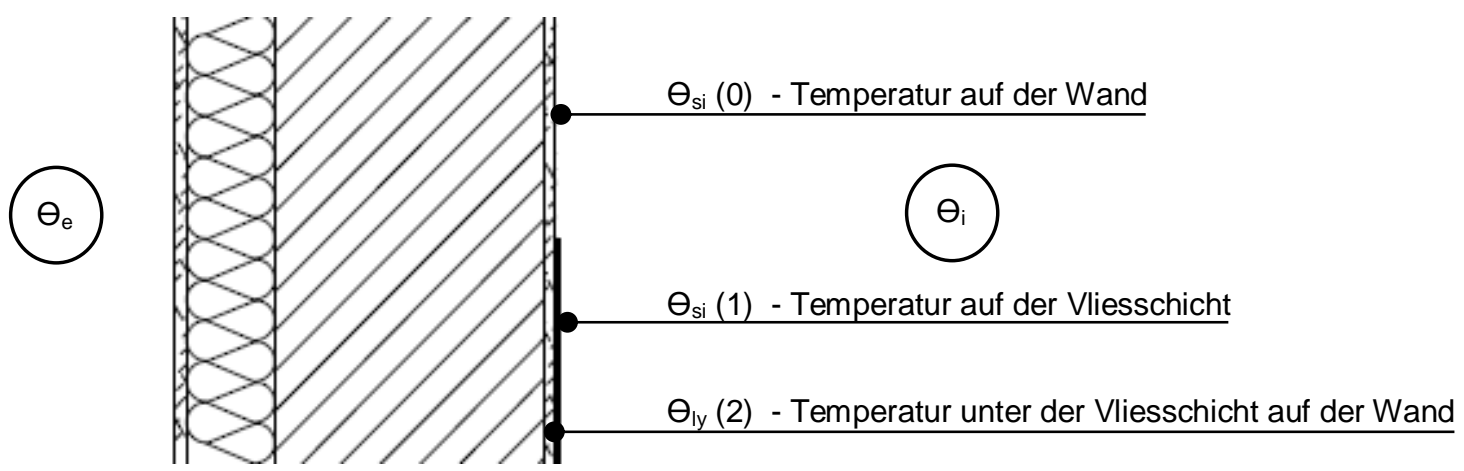

Bauteilschichten: Kunstharzputz, Polystyrolhartschaum, KS-Mauerwerk, Gipsputz, (Vliesschicht)

\section{Raumlufttemperaturverlauf bei einer Raumlufttemperatur-Nachtabsenkung von 2K}

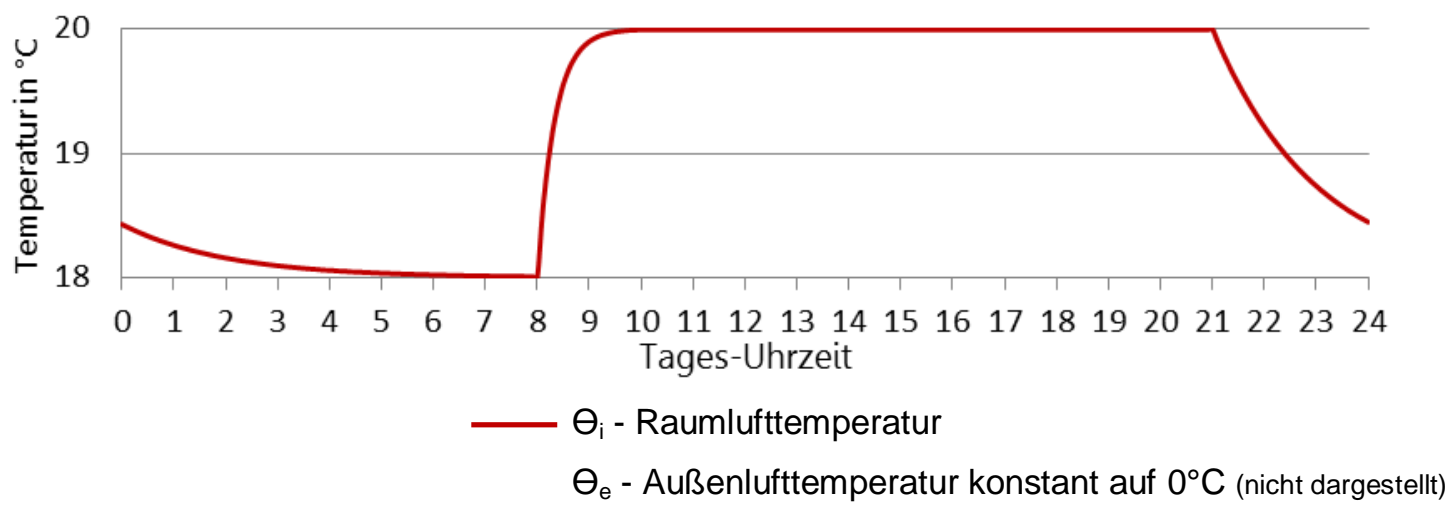

\section{Wandoberflächentemperaturen und Zwischenschichttemperaturen}

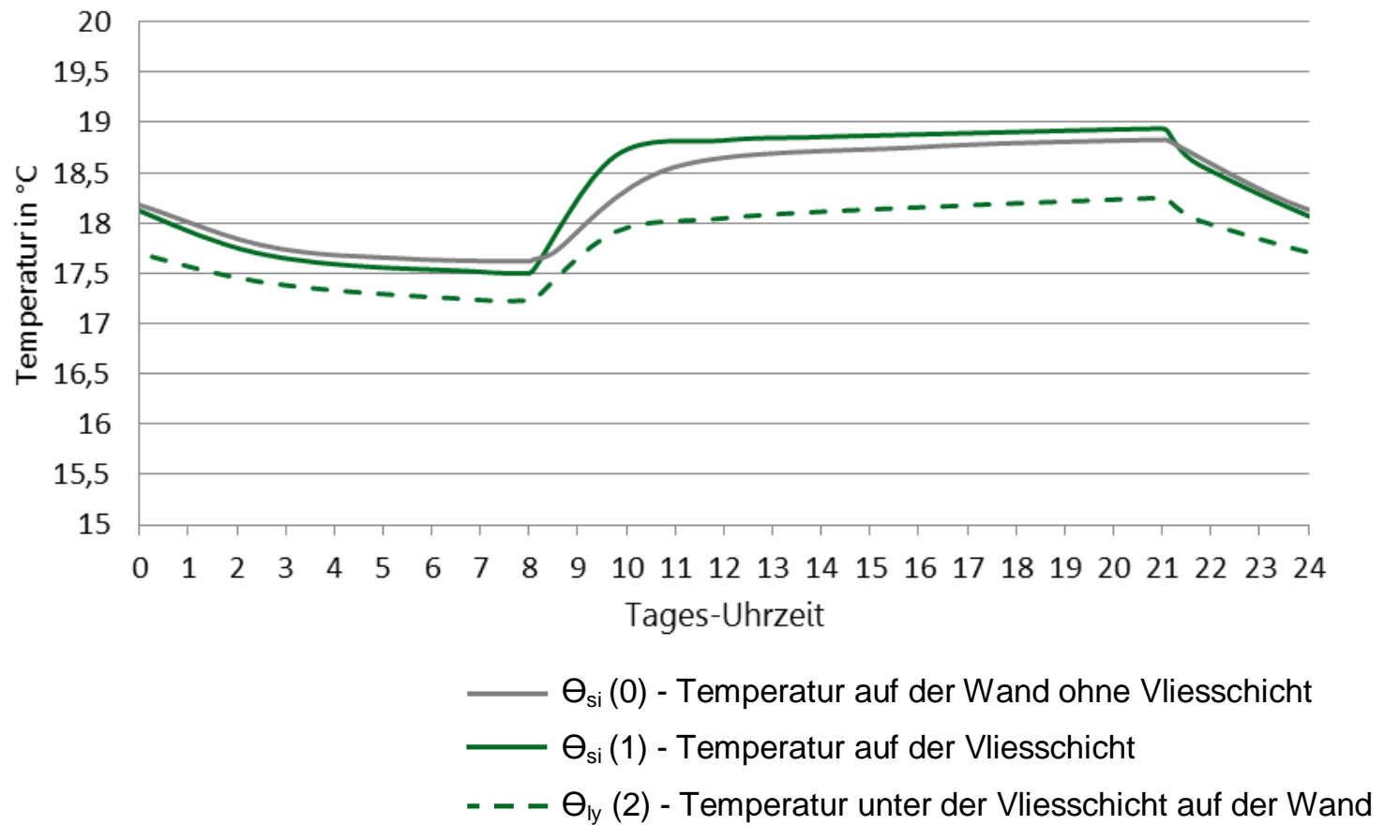

Abbildung 8.11: Wandoberflächentemperaturverläufe für Wandkonstruktion A nach Tabelle 8.10 
B) Kalksandstein-Mauerwerk mit 160 mm Außenwanddämmung

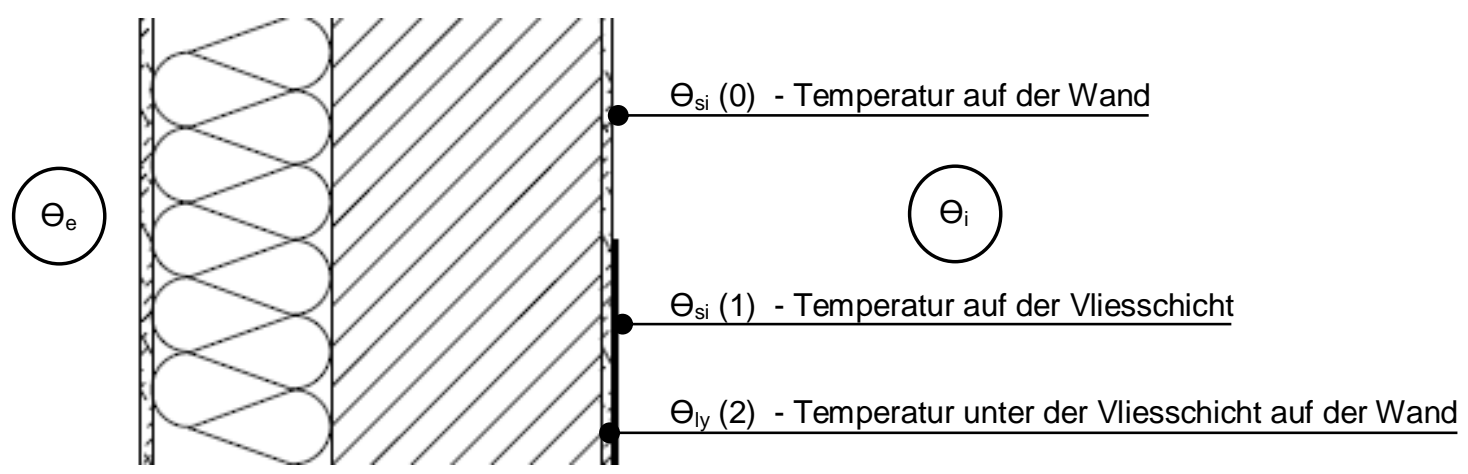

Bauteilschichten: Kunstharzputz, Polystyrolhartschaum, KS-Mauerwerk, Gipsputz, (Vliesschicht)

\section{Raumlufttemperaturverlauf bei einer Raumlufttemperatur-Nachtabsenkung von 2K}

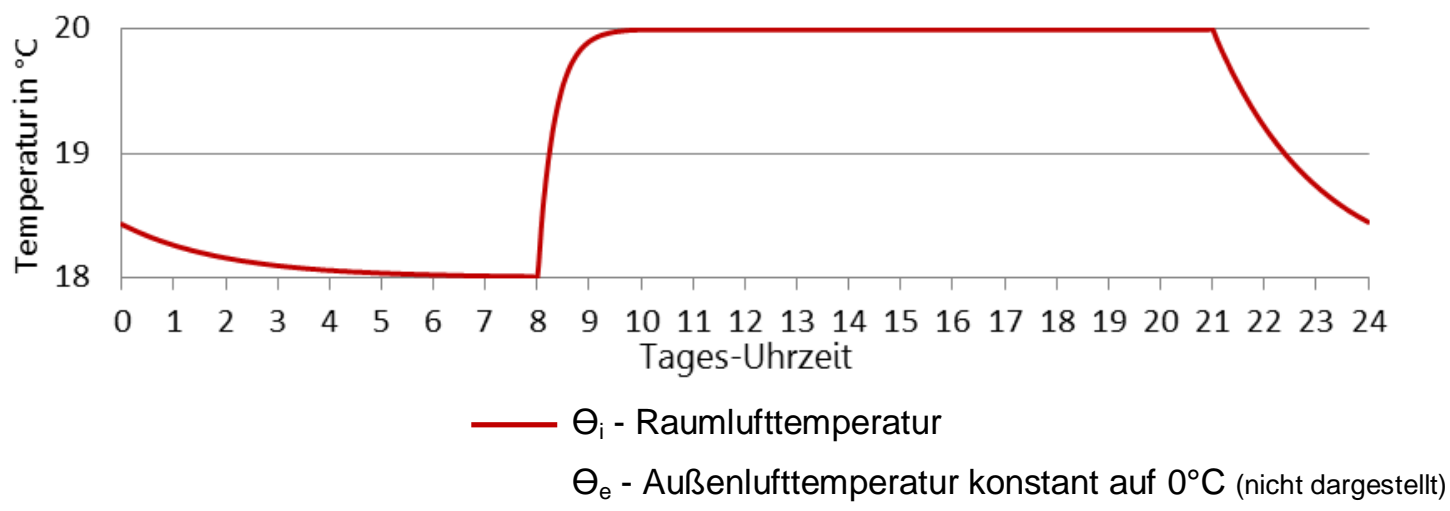

\section{Wandoberflächentemperaturen und Zwischenschichttemperaturen}

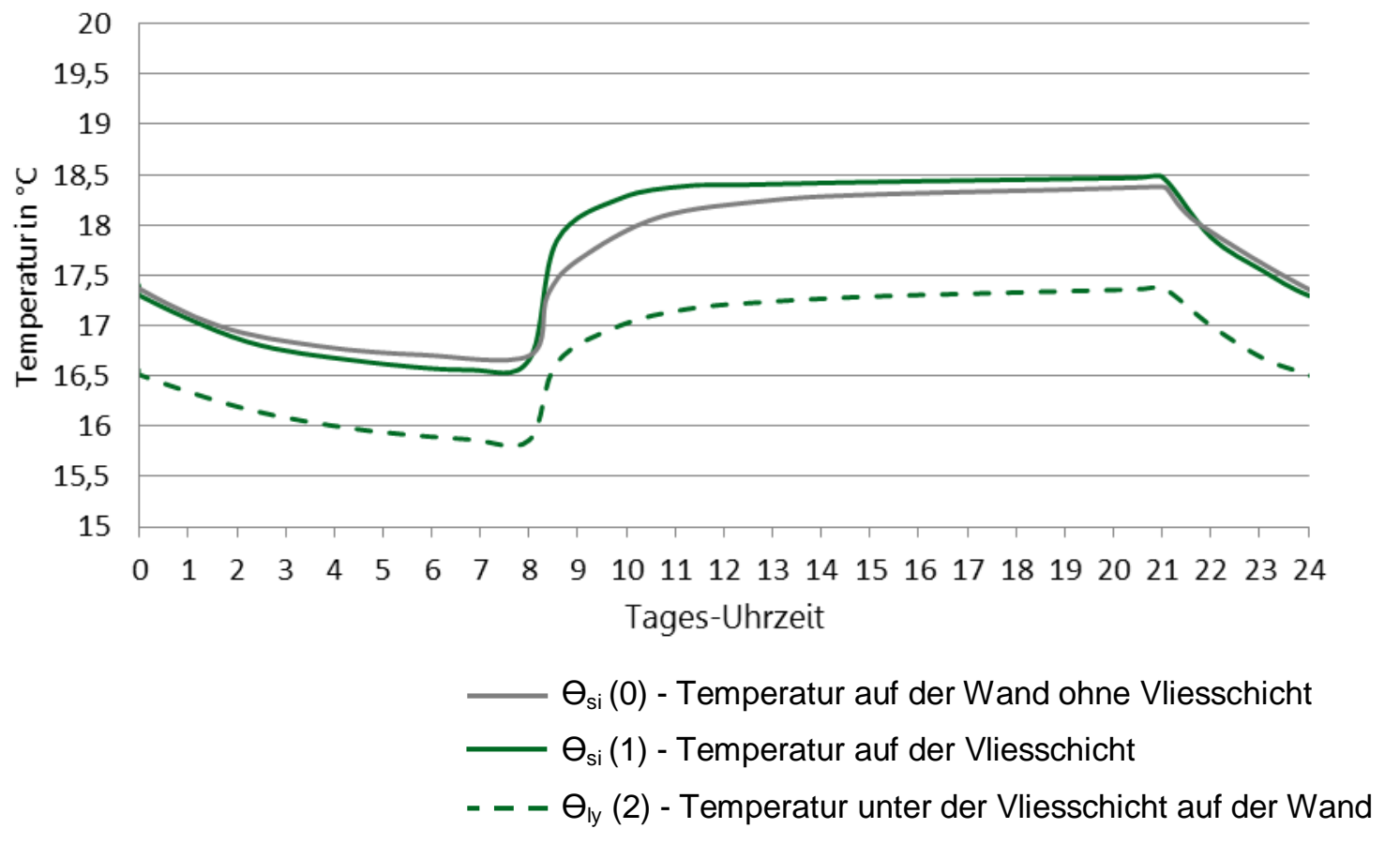

Abbildung 8.12: Wandoberflächentemperaturverläufe für Wandkonstruktion B nach Tabelle 8.10 
C) Kalksandstein-Mauerwerk mit Verblendmauerwerk

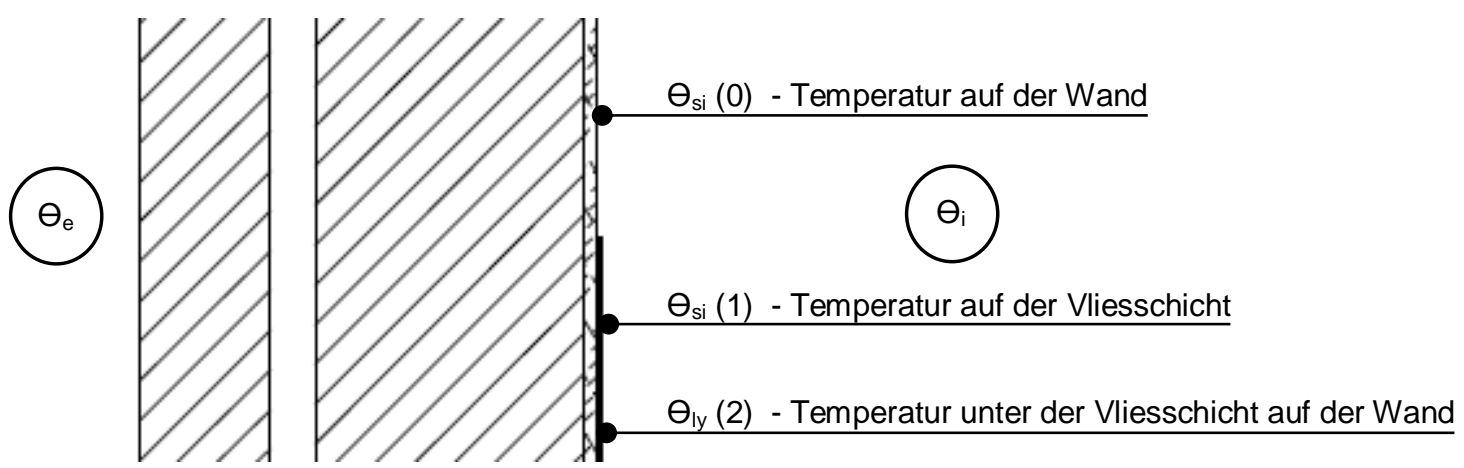

Bauteilschichten: Kunstharzputz, Polystyrolhartschaum, KS-Mauerwerk, Gipsputz, (Vliesschicht)

\section{Raumlufttemperaturverlauf bei einer Raumlufttemperatur-Nachtabsenkung von 2K}

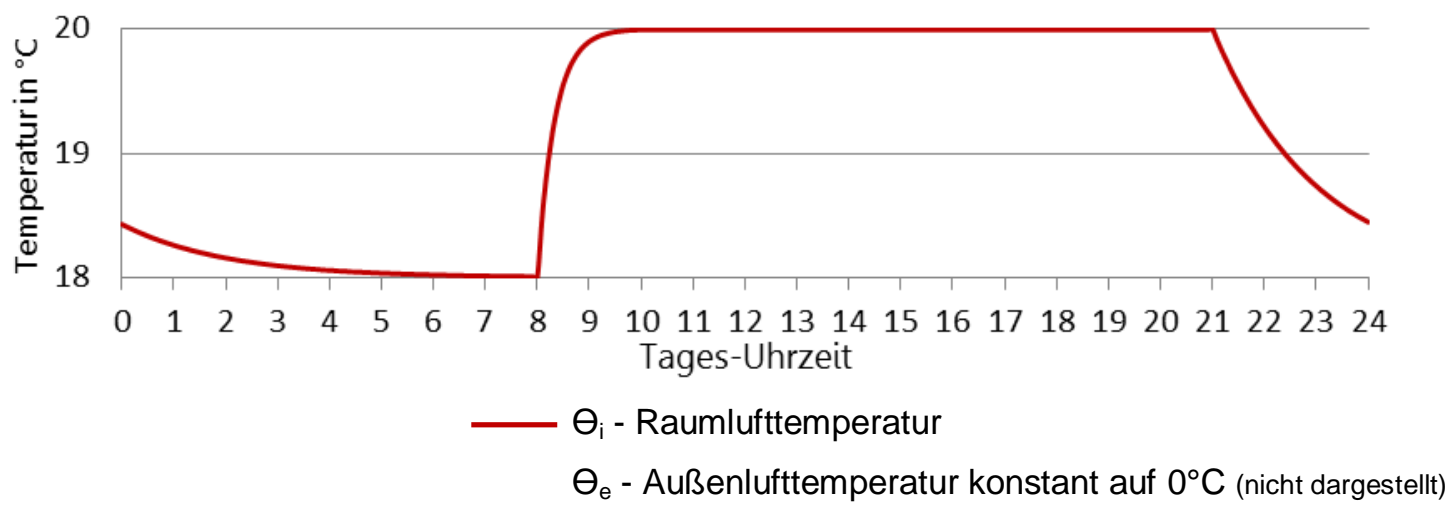

\section{Wandoberflächentemperaturen und Zwischenschichttemperaturen}

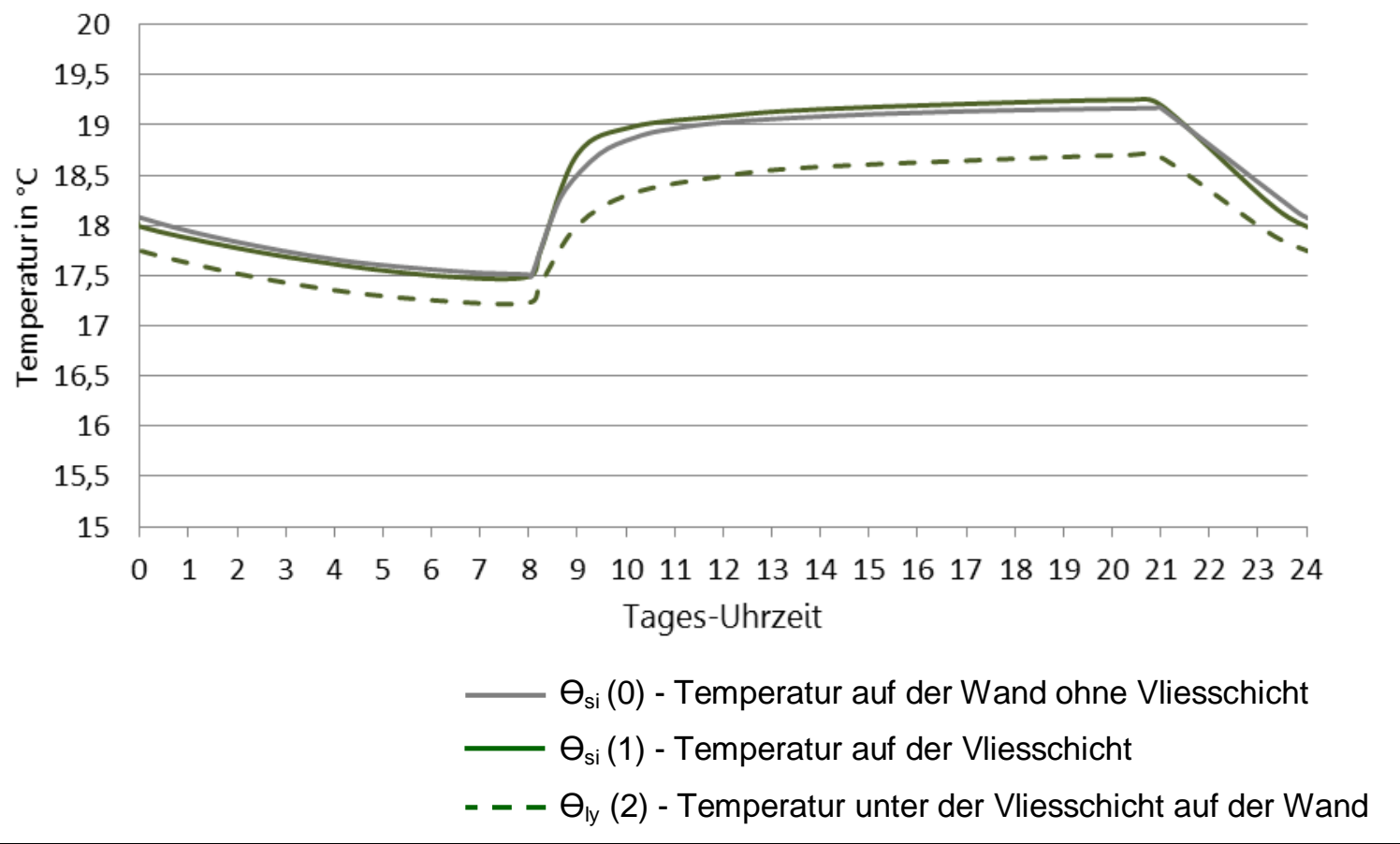

Abbildung 8.13: Wandoberflächentemperaturverläufe für Wandkonstruktion $C$ nach Tabelle 8.10 


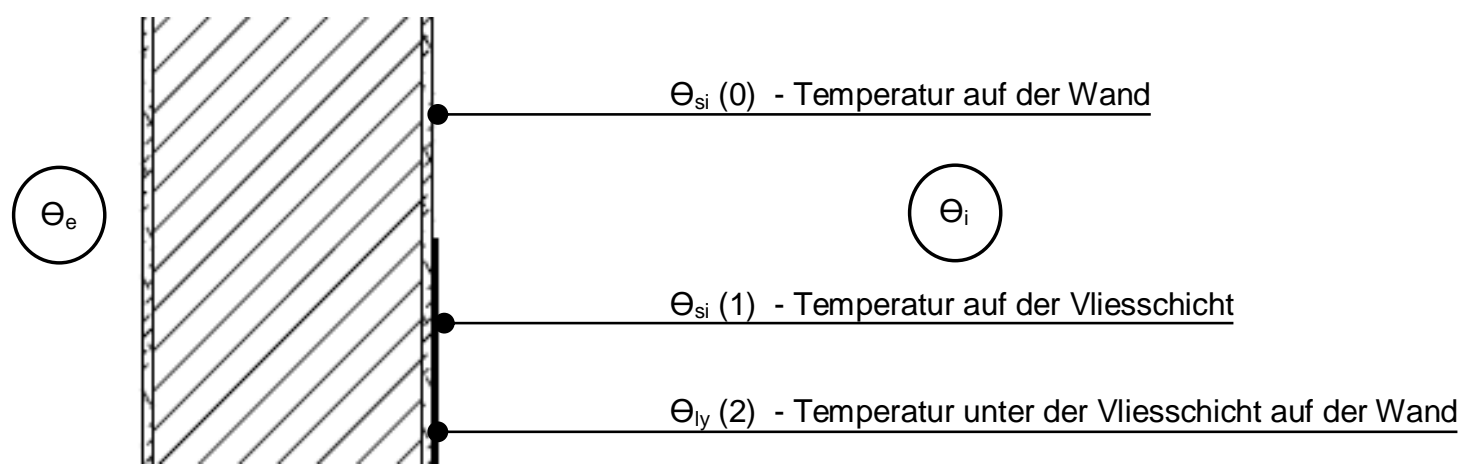

Bauteilschichten: Kunstharzputz, Polystyrolhartschaum, KS-Mauerwerk, Gipsputz, (Vliesschicht)

\section{Raumlufttemperaturverlauf bei einer Raumluftttemperatur-Nachtabsenkung von 2K}

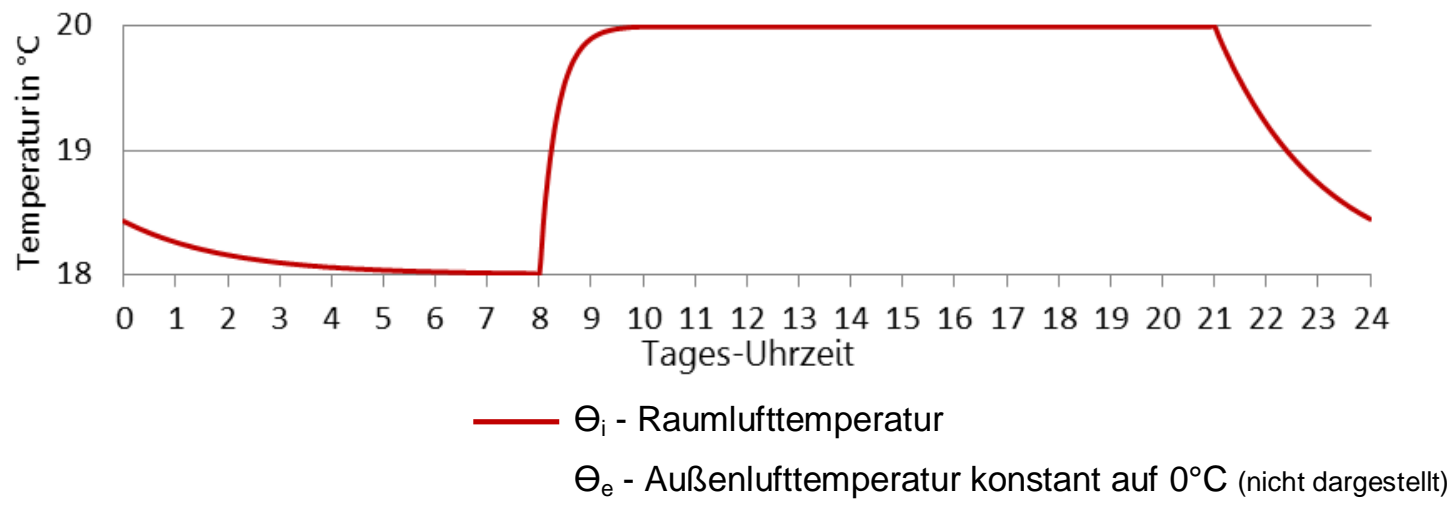

Wandoberflächentemperaturen und Zwischenschichttemperaturen

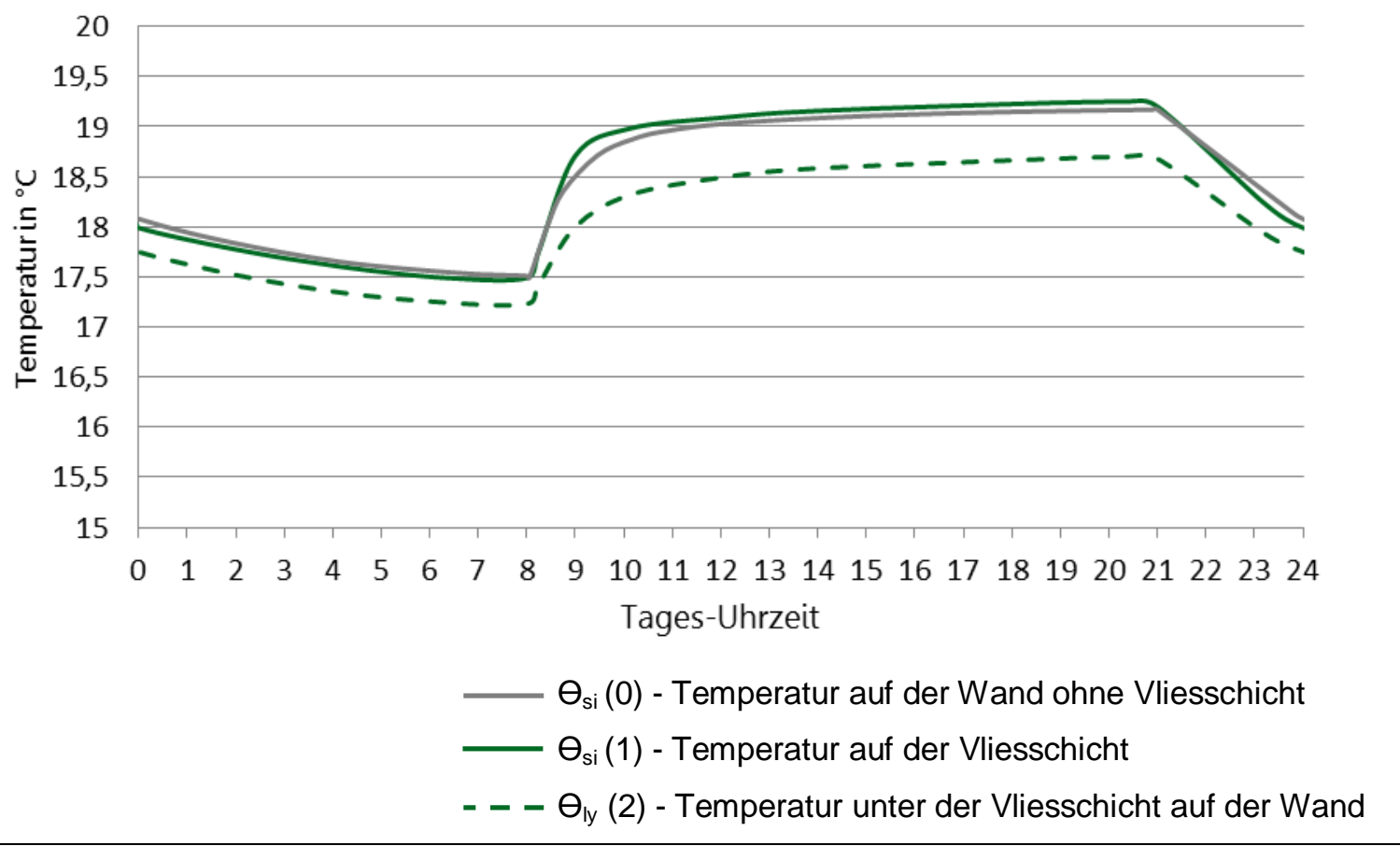

Abbildung 8.14: Wandoberflächentemperaturverläufe für Wandkonstruktion $D$ nach Tabelle 8.10 


\subsection{Berechnung der gespeicherten Energie bei intermittierendem Heizbetrieb}

Für die Berechnung der gespeicherten Energiemengen bei einer intermittierenden Beheizung ist es notwendig, die Wandkonstruktion für die Berechenbarkeit in dünne Schichten zu unterteilen. Von der jeweiligen Schicht kann dann der zeitliche Temperaturverlauf berechnet werden. Bei einer intermittierenden Beheizung sind mehrere Zyklen (Tage) notwendig, bis dass der Bauteilquerschnitt zum jeweiligen Tageszeitpunkt wieder die gleiche Temperatur aufweist. Nach einer entsprechenden Zahl von Zyklen ist dieser eingeschwungene Zustand erreicht.

Im Folgenden wird die Wandkonstruktion D aus dem Kapitel 8.3 untersucht, siehe Abbildung.

Die Ergebnisse für die anderen Innen- und Außenwandkonstruktionen aus dem Kapitel 8.3 und 8.4 sind tabellarisch in einer Kurzform in Kapitel 8.5.1 dargestellt.

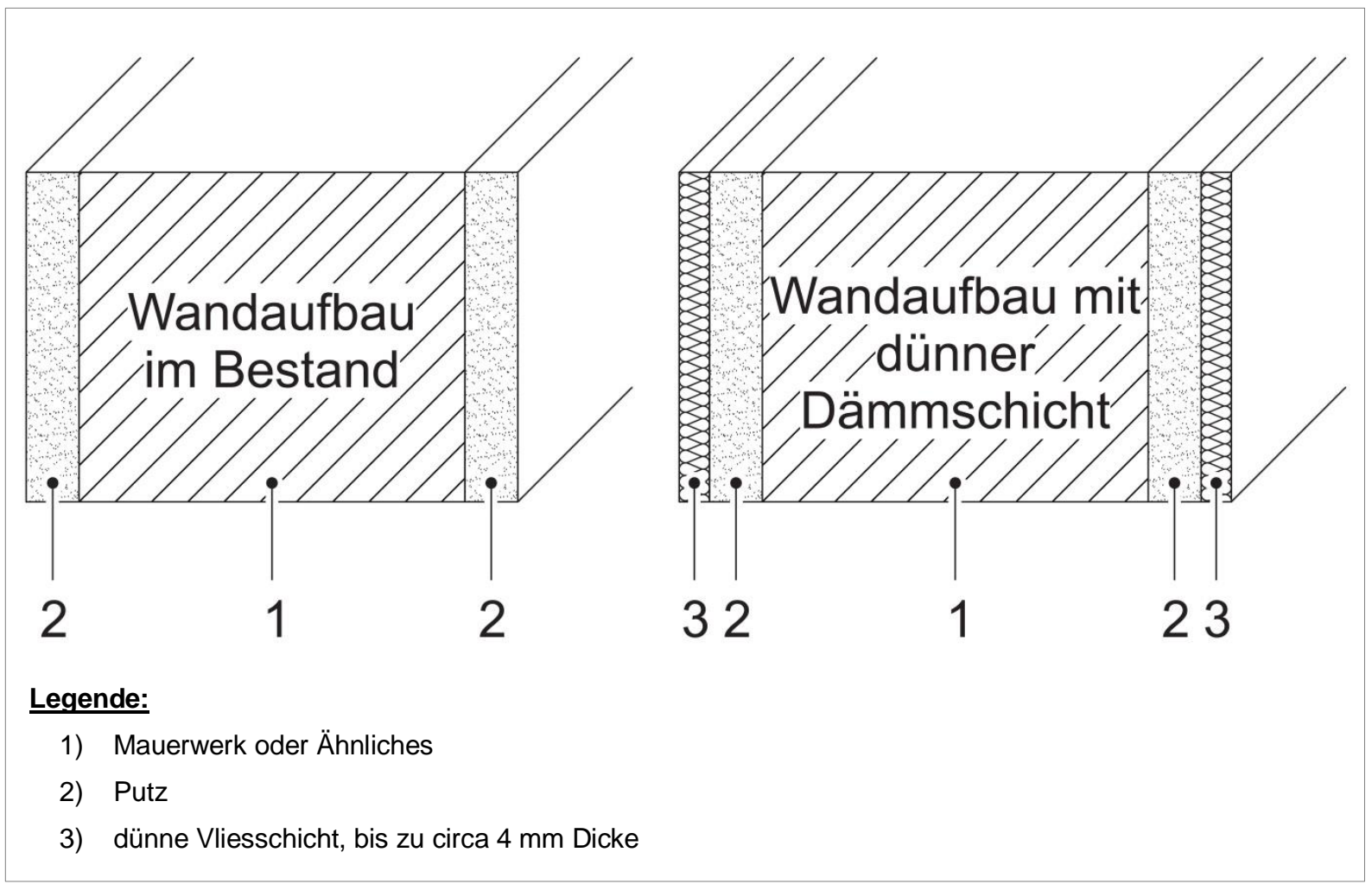

Abbildung 8.15: Schnittzeichnung der rechnerisch untersuchten Wandkonstruktion D aus Kapitel 8.3 
Die Raumlufttemperaturen sind auf beiden Wandseiten mit den Raumlufttemperaturen aus Abbildung 8.16 angenommen worden.

Ausführlich wird die Raumlufttemperaturkurve mit einem Temperaturabfall von 2 Kelvin betrachtet. Die Ergebnisse mit anderen Temperaturkurven, das heißt mit 3 Kelvin bzw. 4 Kelvin Raumlufttemperaturabfall, sind in einer Kurzform in Kapitel 8.5.1 dargestellt.

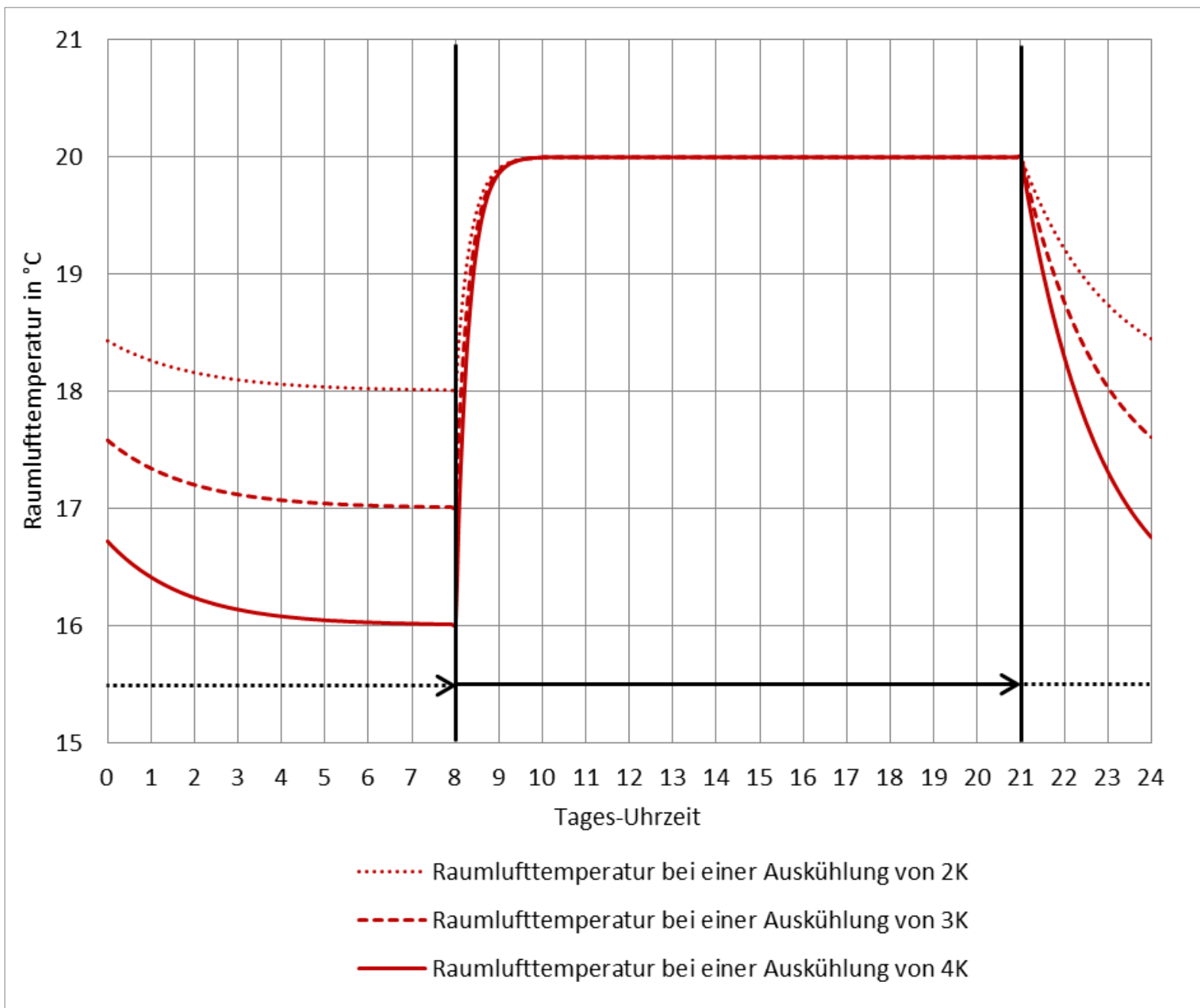

Abbildung 8.16: Angenommene Raumlufttemperaturen abhängig von der Tageszeit

Aus den Temperaturverläufen sind die minimalen und maximalen Temperaturen im eingeschwungenen Zustand über den Wandquerschnitt ermittelt und in Abbildung 8.17 dargestellt worden. 


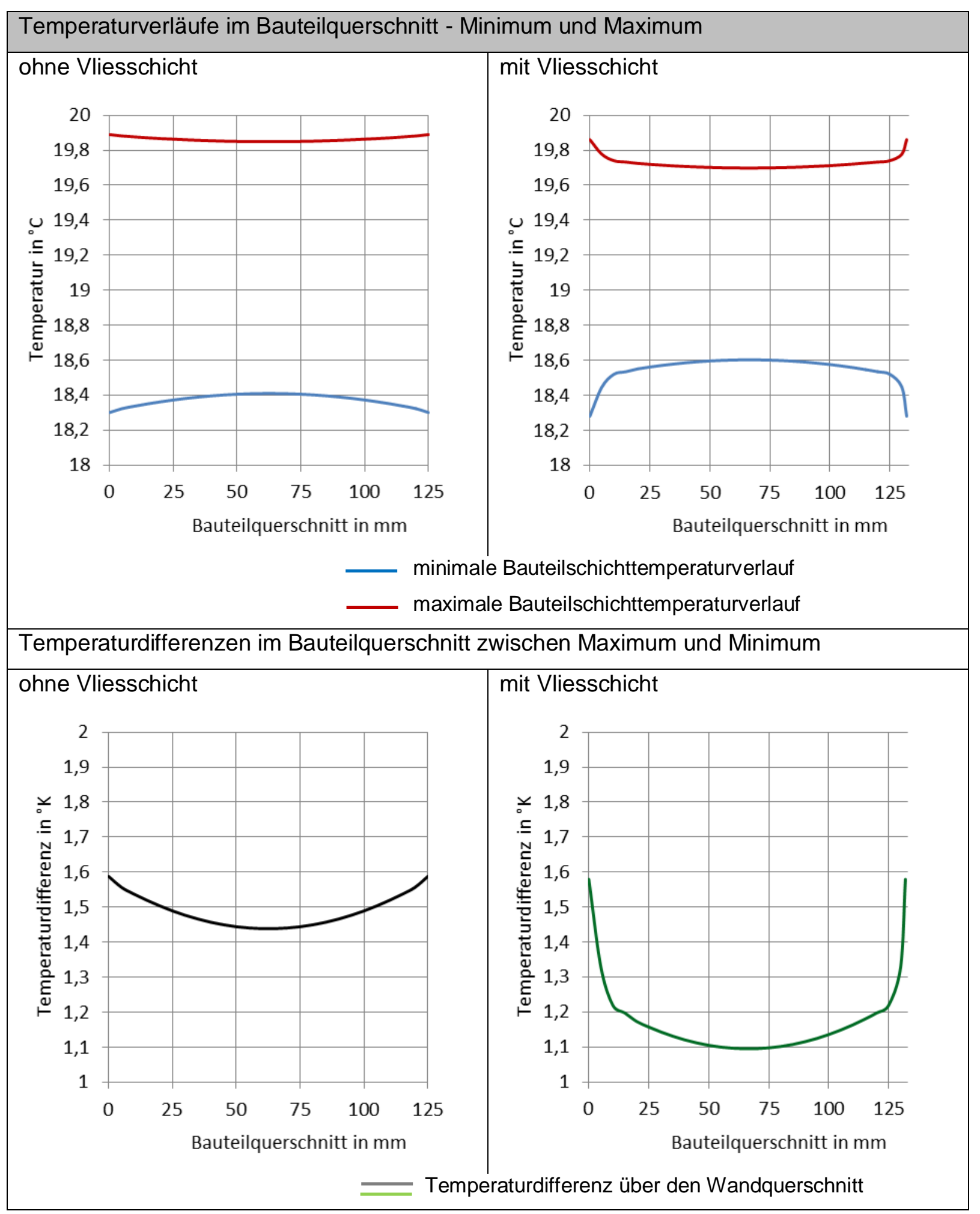

Abbildung 8.17: Minimal- und Maximaltemperaturverläufe sowie deren Differenzen über den Wandquerschnitt einer Innenwand nach Abbildung $\mathbf{8 . 1 5}$ bei der festgelegten intermittierenden Beheizung 
Stellt man die Formel 3.1 nach $\Delta Q$ um, kann man die Wärmemengen für den Wärmespeichervorgang berechnen, vergleiche Formel 8.1. Zur Berechnung der Wärmemengen in den Bauteilschichten, wie in Abbildung 8.18, muss jetzt nur die jeweilige Temperaturdifferenz der Schicht mit der Masse und der spezifischen Wärmekapazität multipliziert werden.

$\Delta Q=m * c * \Delta T$

Formel 8.1: Wärmemenge abhängig vom Stoff und einer Temperaturdifferenz vor und nach der Wärmeaufnahme

mit:

$\begin{array}{lll}\mathrm{C} & {\left[\frac{\mathrm{J}}{\mathrm{kg} \cdot \mathrm{K}}\right]} & \text { spezifische Wärmekapazität der jeweiligen Schicht } \\ \Delta \mathrm{Q} & {\left[\frac{\mathrm{J}}{\mathrm{m}^{2}}\right]} & \text { Wärmemengendifferenz der jeweiligen Schicht } \\ \mathrm{m} & {[\mathrm{kg}]} & \text { Masse der jeweiligen Schicht } \\ \Delta \mathrm{T} & {[\mathrm{K}]} & \text { Temperaturdifferenz der jeweiligen Schicht }\end{array}$




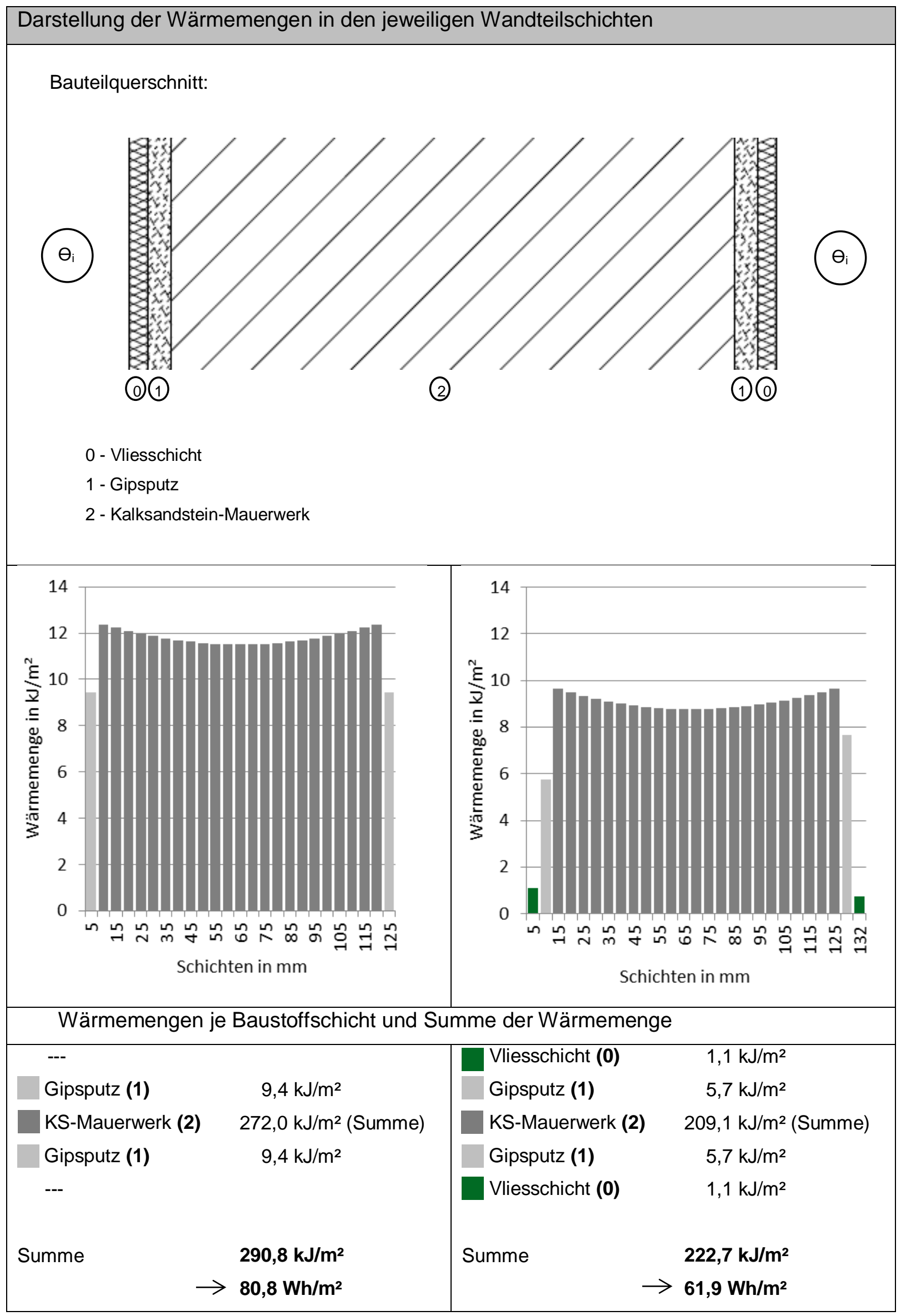

Abbildung 8.18: Wärmemengen in den Teilschichten und in der Gesamtwand mit und ohne Vliesschicht 
Aus Abbildung 8.18 geht hervor, welche Energiemengen in den einzelnen Schichten und in der Gesamtkonstruktion bei einer intermittierenden Beheizung über den Tag gespeichert und wieder abgegeben werden.

Der thermische Unterschied zwischen der Wand mit und ohne Vliesschicht scheint auf den ersten Blick nicht sonderlich hoch zu sein. Betrachtet man jedoch die gesamte Wandfläche eines Raumes, so wird sehr viel an Energie für das Aufheizen verwendet. Zudem muss diese Energiemenge bei einer üblichen Nachtabsenkung an jedem Tag in der Heizperiode aufgebracht werden.

\subsubsection{Gespeicherte Energiemenge bei intermittierendem Heizbetrieb}

Es wurden die Wärmemengen für die Innenwandkonstruktionen nach Kapitel 8.2 bei den Temperaturverläufen nach Abbildung 8.6 berechnet.

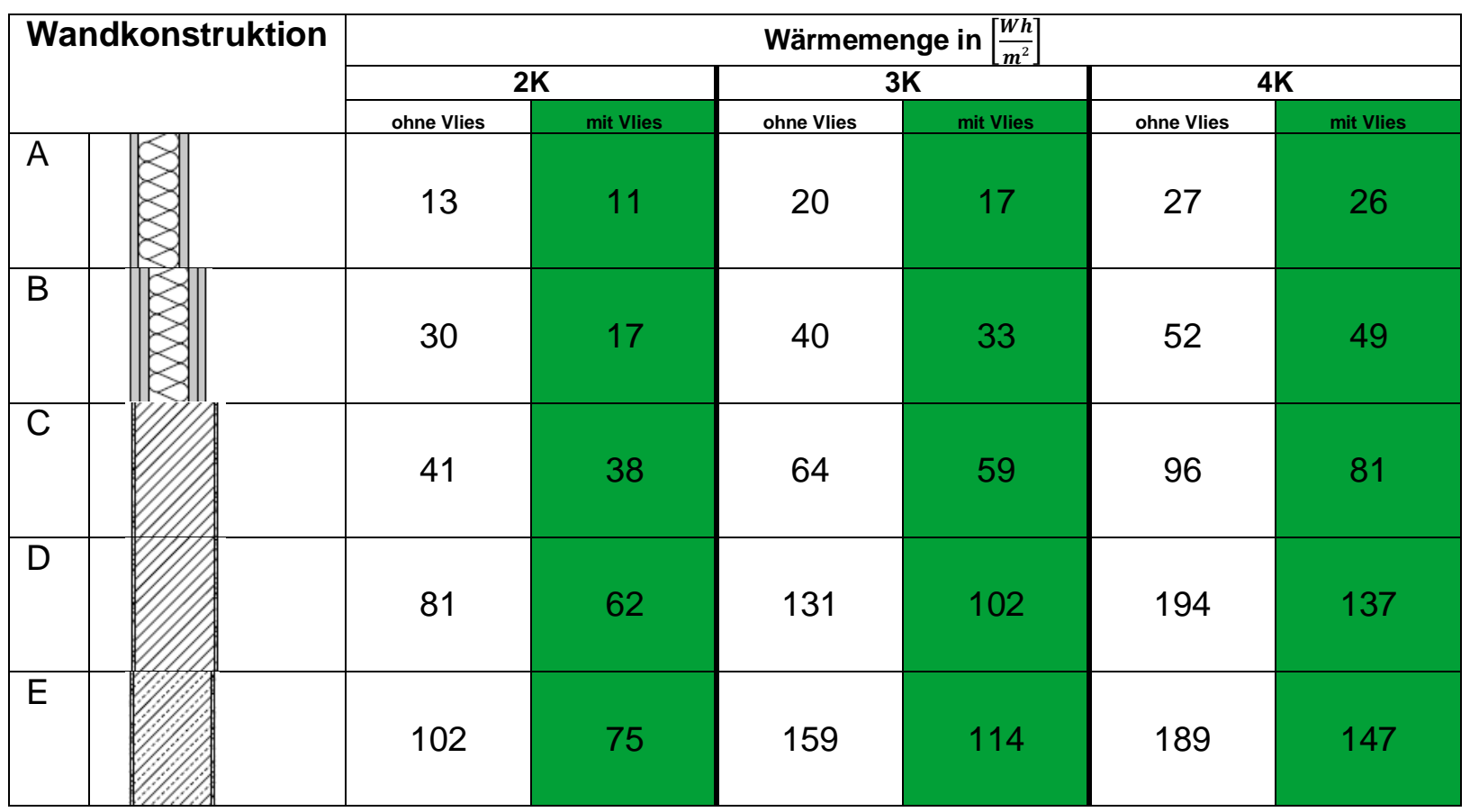

Abbildung 8.19: Gespeicherte Wärmemengen bei einer intermittierenden Beheizung für verschiedene Innenwandwandkonstruktionen und Temperaturverläufe 


\subsection{Ergebnisdiskussion}

Mittels messtechnischen und rechnerischen Ermittlungen wurde das thermische Verhalten von raumumschließenden Wänden bei einer intermittierenden Raumbeheizung, welche in Wohn- und Arbeitsbereichen normal ist, analysiert. Dabei wurde insbesondere der Einfluss von dünnen (bis zu $4 \mathrm{~mm}$ dicken) wärmedämmenden Wandbeschichtungen auf üblichen Wänden im Vergleich zu normal ausgebildeten Wänden untersucht und bewertet. Die Arbeitshypothese, dass dünne wärmedämmende Wandbeschichtungen das thermische Raumklima günstig beeinflussen und zudem bei einer intermittierenden, üblichen Beheizung von Räumen Heizenergie eingespart werden kann, hat sich bestätigt.

Es konnte messtechnisch wie auch rechnerisch nachgewiesen werden, dass durch diese Maßnahme der Wärmespeicher „Wand“ beispielsweise bei einer RaumlufttemperaturNachtabsenkung weniger Wärme verliert und somit eine raschere Wiederaufheizung möglich wird. Eine tapezierte (bis zu $4 \mathrm{~mm}$ dicke) Vliesschicht wirkt zudem als leicht temperierbare Membran, wodurch die Raumaufheizung beschleunigt wird, vergleiche Kapitel 8.4.

Interessant sind die Berechnungsergebnisse bei einer Außenwandkonstruktion mit einem Wärmedämmverbundsystem, vergleiche Abbildung 8.11 und 8.12. Hier wird ersichtlich, dass auch bei einem guten Dämmniveau die Trägheit der Wandkonstruktion auf die raumseitige Oberflächentemperatur einen nennenswerten Einfluss hat. Somit sind auch bei einer intermittierenden Beheizung ein günstiges thermisches Raumklima und eine Energieeinsparung zu erwarten.

Die Berechnung der Wärmemengen, welche in einem Tages-Beheizungszyklus bei einer Nacht-Heizungsunterbrechung abgegeben und aufgenommen werden müssen zeigt, dass je nach „Oberflächenbehandlung“ der raumumschließenden Wände mehr oder weniger Energie für den Wiederaufheizvorgang zur Verfügung gestellt werden muss. 


\section{Diskussion der Ergebnisse}

\subsection{Temperierung von realen Wohn- und Büroräumen}

Die Objektuntersuchungen aus Kapitel 6 haben gezeigt, dass ein kontinuierlicher Heizbetrieb in den untersuchten Wohn- und Büroräumen nicht vorhanden ist. Bei Büroräumen kommt neben der Heizungsunterbrechung in der Nachtzeit auch eine Heizungsunterbrechung über das Wochenende hinzu, vergleiche Kapitel 6.1 und 6.2.

In Abbildung 9.1 sind für die untersuchten Räume R1 bis R13 die jeweilige Minimum-, Maximum- und mittlere Raumlufttemperatur dargestellt.

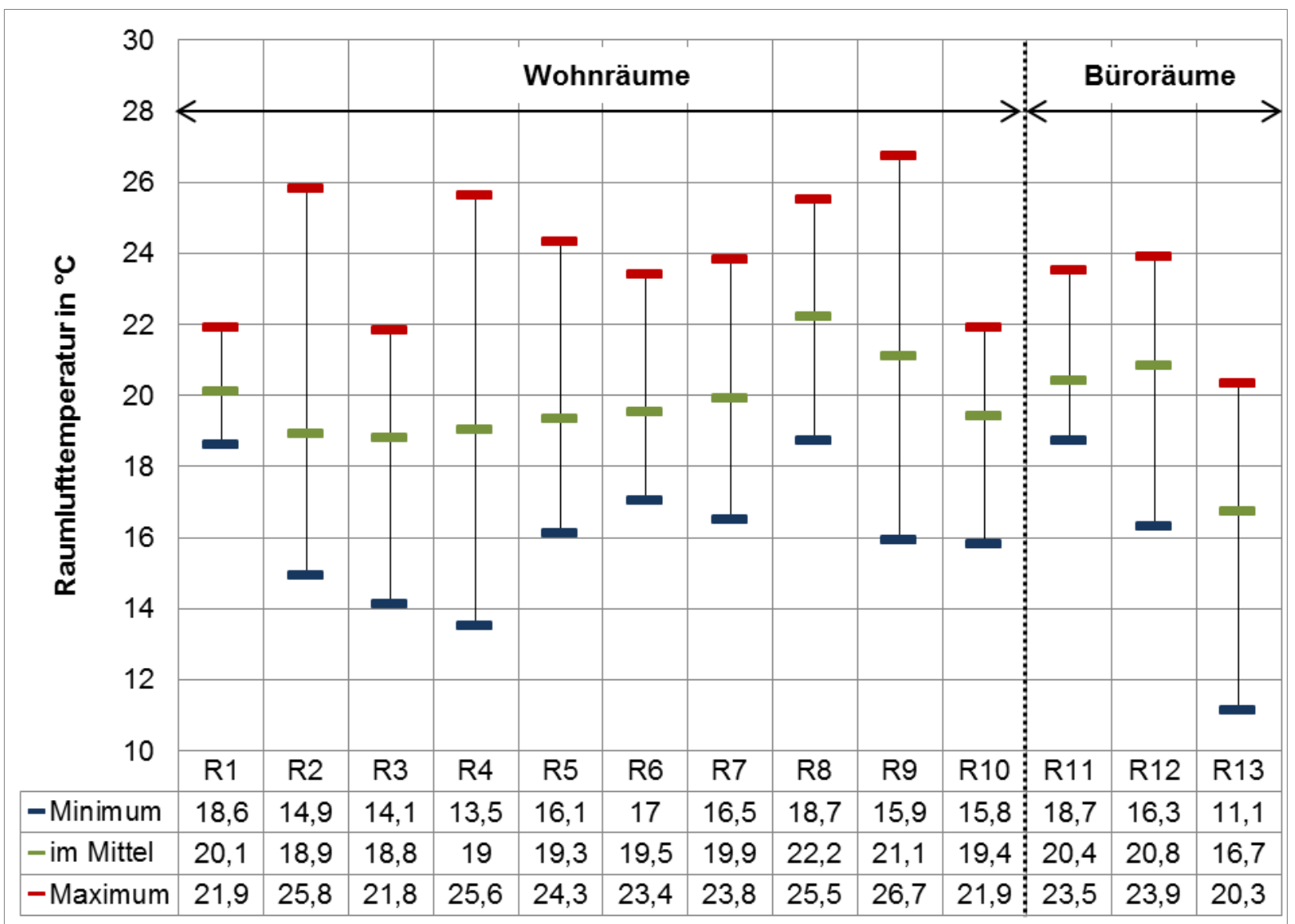

Abbildung 9.1: Minimum-, Maximum- und mittlere Raumlufttemperaturen der untersuchten Räume

Es ist ersichtlich, dass Raumlufttemperaturveränderungen in den meisten Räumen vorhanden sind.

Diese aufgezeichneten Raumlufttemperaturkurven spiegeln das Nutzerverhalten wieder und sind für die weiteren Untersuchungen der Oberflächentemperaturen bei einer intermittierenden Beheizung von großer Bedeutung. Durch das ermittelte Benutzerprofil zeigt sich, ob eine 
intermittierende Beheizung für das Objekt angesetzt werden kann. Bei einer intermittierenden Beheizung und einer massiven Bauweise ist eine Betrachtung der inneren Speichermassen lohnend.

\subsection{Messtechnisch und rechnerisch untersuchter Einfluss dünner Dämmschichten an Wandkonstruktionen}

In Kapitel 7 sind Versuche im Labor-Prüfstand und im Objekt durchgeführt worden. Hier ist der Einfluss einer dünnen Dämmschicht auf eine massive Wandkonstruktion messtechnisch ermittelt worden. Die Versuche aus dem Labor-Prüfstand sind in Kapitel 8.2 mit Hilfe der Software "HeatTransmisson“ validiert worden. Die Abweichungen zwischen den gemessenen Versuchsergebnissen und den Rechenergebnissen sind vernachlässigbar klein, so dass eine Berechnung mit Hilfe der Software für andere Wandkonstruktionen und andere Temperaturparameter durchführbar ist. In Kapitel 8.3 sind daraufhin verschiedene Innenwandkonstruktionen und in Kapitel 8.4 verschiedene Außenwandkonstruktionen mit Hilfe der Software „HeatTransmission“ betrachtet worden.

Die Ergebnisse zeigen, dass die Oberflächentemperatur bei einer massiven Wandkonstruktion mit einer dünnen Dämmschicht während der Anheizphase deutlich höher ist als bei einer Wandkonstruktion ohne Dämmschicht.

Für die Praxis bedeutet dies, dass die Behaglichkeit im Raum während der Anheizphase schneller hergestellt werden kann, da die operative Temperatur die Mittelungstemperatur aus Oberflächentemperatur und Lufttemperatur darstellt.

Dies wurde auch durch die Ergebnisse aus dem Laborversuch in Kapitel 7.1.1 bei einer einmaligen Anheizung von $12^{\circ} \mathrm{C}$ nach $21^{\circ} \mathrm{C}$ nachgewiesen. In Tabelle 9.2 ist der Zeitraum zum Erreichen der operativen Temperatur von $19,0^{\circ} \mathrm{C}, 19,5^{\circ} \mathrm{C}$ und $20,0^{\circ} \mathrm{C}$ mit den verschiedenen Wandbelägen dargestellt.

\begin{tabular}{|l|c|c|c|}
\hline \multirow{2}{*}{ Wandbeschichtungen } & \multicolumn{3}{|c|}{ Zeitraum zur Erreichen der operativen Temperatur } \\
\cline { 2 - 4 } & $\mathbf{1 9 , 0}{ }^{\circ} \mathbf{C}$ & $\mathbf{1 9 , 5}{ }^{\circ} \mathbf{C}$ & $\mathbf{2 0 , 0}^{\circ} \mathbf{C}$ \\
\hline $0 /$ ohne Belag & $17,9 \mathrm{~h}$ & $23,8 \mathrm{~h}$ & $35,9 \mathrm{~h}$ \\
\hline $1 / 1,2 \mathrm{~mm}$ Vliesschicht & $11,4 \mathrm{~h}$ & $15,5 \mathrm{~h}$ & $21,7 \mathrm{~h}$ \\
\hline $2 / 2,4 \mathrm{~mm}$ Vliesschicht & $9,1 \mathrm{~h}$ & $11,3 \mathrm{~h}$ & $14,0 \mathrm{~h}$ \\
\hline $3 / 3,6 \mathrm{~mm}$ Vliesschicht & $6,7 \mathrm{~h}$ & $8,3 \mathrm{~h}$ & $10,3 \mathrm{~h}$ \\
\hline $4 / 30 \mathrm{~mm}$ Polystyrol & $1,9 \mathrm{~h}$ & $2,2 \mathrm{~h}$ & $2,6 \mathrm{~h}$ \\
\hline
\end{tabular}

Tabelle 9.2: Zeitraum bis zum Erreichen der jeweiligen operativen Temperatur von $19,0^{\circ} \mathrm{C}, 19,5{ }^{\circ} \mathrm{C}$ und $20^{\circ} \mathrm{C}$ bei einer einmaligen Beheizung von $12,0^{\circ} \mathrm{C}$ nach $21,0^{\circ} \mathrm{C}$ im Laborprüfstand 
Die Ergebnisse aus den Laborversuchen nach Kapitel 7.1.2, bei einer intermittierenden Beheizung zwischen $17,0^{\circ} \mathrm{C}$ und $21,0^{\circ} \mathrm{C}$, zeigen ebenfalls der Vorteil bei der Wiederanheizung zur Erreichung der operativen Temperatur von $19,0^{\circ} \mathrm{C}, 19,5^{\circ} \mathrm{C}$ und $20,0^{\circ} \mathrm{C}$, vergleiche Tabelle 9.3.

\begin{tabular}{|l|c|c|c|}
\hline \multirow{2}{*}{ Versuch } & \multicolumn{3}{|c|}{ Operative Temperatur } \\
\cline { 2 - 4 } & $\mathbf{1 9 , 0}{ }^{\circ} \mathbf{C}$ & $\mathbf{1 9 , 5}{ }^{\circ} \mathbf{C}$ & $\mathbf{2 0 , 0}^{\circ} \mathbf{C}$ \\
\hline $0 /$ ohne Belag & $3,6 \mathrm{~h}$ & $9,3 \mathrm{~h}$ & wird nicht erreicht \\
\hline $1 / 1,2 \mathrm{~mm}$ Vliesschicht & $1,8 \mathrm{~h}$ & $5,3 \mathrm{~h}$ & $15,6 \mathrm{~h}$ \\
\hline $2 / 2,4 \mathrm{~mm}$ Vliesschicht & $2,0 \mathrm{~h}$ & $5,3 \mathrm{~h}$ & $15,1 \mathrm{~h}$ \\
\hline $3 / 3,6 \mathrm{~mm}$ Vliesschicht & $1,6 \mathrm{~h}$ & $4,4 \mathrm{~h}$ & $9,6 \mathrm{~h}$ \\
\hline $4 / 30 \mathrm{~mm}$ Polystyrol & $1,4 \mathrm{~h}$ & $1,9 \mathrm{~h}$ & \\
\hline
\end{tabular}

Tabelle 9.3: Dauer bis zum Erreichen der jeweiligen operativen Temperatur von $19,0^{\circ} \mathrm{C}, 19,5^{\circ} \mathrm{C}$ und $20^{\circ} \mathrm{C}$ bei einer intermittierenden Beheizung zwischen $17,0^{\circ} \mathrm{C}$ und $21,0^{\circ} \mathrm{C}$ im Laborprüfstand

Ein Problem bei der Versuchsdurchführung sowie der rechnerischen Ermittlung mit Hilfe der Software „HeatTransmission“ ist, dass die Temperaturkurve der Auskühlung sowie der Anheizung erzwungen ist. Hierdurch sind, Einflüsse die durch schnellere Anheizbarkeit der Wandoberfläche auf die Raumlufttemperatur nicht berücksichtigt. Für die Aussage der gewonnenen Ergebnisse bedeutet dies, dass mit entsprechender Wandbeschichtung die Anheizzeit geringer wird als hier berechnet und im Laborprüfstand ermittelt, da die Temperatur der Raumluft von der Wandoberflächentemperatur mitbestimmt wird.

Zur Vergleichbarkeit der Wandkonstruktionen ist die hier durchgeführte Betrachtung geeignet, da Einflüsse von Raumgröße und Art der Beheizung unberücksichtigt bleiben.

In den Versuchsobjekten aus Kapitel 7.3 und 7.4 sind die Messungen unter natürlichen Bedingungen durchgeführt. Hier sind die Einflüsse der Raumgröße und des Beheizungssystems vorhanden.

Die erzielte Energieeinsparungen für das Pilotobjekt aus Kapitel 7.4 sind bei dem geringen Umfang der baulichen Veränderung, hier eine dünne Dämmschicht von $4 \mathrm{~mm}$ an den Wänden beachtlich, vergleiche Tabelle 9.4.

\begin{tabular}{|c|c|c|c|c|c|}
\hline Heizperiode: & $2006-2007$ & $2007-2008$ & $2008-2009$ & $2009-2010$ & $2010-2011$ \\
\hline $\begin{array}{c}\text { Jahresverbrauch in } \\
\mathrm{kWh} / \mathrm{a}\end{array}$ & $\begin{array}{c}9260 \\
(100 \%)\end{array}$ & $\begin{array}{c}9593 \\
(104 \%)\end{array}$ & $\begin{array}{l}8230^{*} \\
(99 \%)\end{array}$ & $\begin{array}{l}7621 \\
(82 \%)\end{array}$ & $\begin{array}{l}7732 \\
(84 \%)\end{array}$ \\
\hline
\end{tabular}

Tabelle 9.4: Energieverbräuche des Pilotobjekts, welches in Kapitel 7.4 genauer dargestellt wurde 
Die Energieeinsparungen beziehen sich auf das hier untersuchte Gebäude mit dem vorhandenen Nutzerprofil. Eine pauschale Übertragung dieser Einsparungen auf andere Objekte ist nicht möglich. Vielmehr ist vorab immer eine individuelle Objekt- und Nutzeranalyse notwendig, bevor Aussagen zu möglichen Optimierungsmaßnahmen und den daraus resultierenden Energieeinsparungen getroffen werden können. 


\section{Zusammenfassung}

\subsection{Untersuchungsergebnisse}

Die aufgezeichneten Raumlufttemperaturen aus mehreren Räumen zeigen, dass eine intermittierende Beheizung in Wohn- und Arbeitsräumen vorhanden ist, vergleiche Kapitel 6.

Bei einer intermittierenden Beheizung sind die Betrachtung der Speichermassen der Bauteilkonstruktionen, die Lage der Schichten sowie die thermischen Parameter für die Auskühlund Anheizprozesse von Bedeutung.

Die Mess- und Berechnungsergebnisse zeigen, dass sehr dünne wärmedämmende Beschichtungen von bis zu $4 \mathrm{~mm}$ an den jeweiligen Randschichten von Innen- und Außenwänden eine deutliche Verkürzung der Anheizphase bewirken. Der Unterschied zwischen den verschiedenen Dämmstoffdicken ist aus den Versuchsergebnissen in Kapitel 6.2 bis 6.4 erkennbar. Diese Beschichtung führt zu einer deutlichen Verkürzung der Anheizphase, wie die Versuchsergebnisse zeigen - und dies auch schon bei sehr geringen Dämmschichtdicken (von $1 \mathrm{~mm}$ ) an Wandoberflächen, vergleiche Kapitel 7.1.1 und 7.1.2. Neben der Verkürzung der Anheizphase wird die Auskühlung von massiven Bauteilen im Vergleich zu dickeren Dämmstoffdicken beziehungsweise Bauteilen ohne Dämmschichten verringert, wie dies beispielsweise aus dem Vergleich der Messergebnisse mit einer $30 \mathrm{~mm}$ dicken Polystyroldämmschicht zu erkennen ist, vergleiche Kapitel 7.1.1.

\subsection{Vorteile für das Raumklima bei entsprechenden Maßnahmen}

Vliesbeschichtungen verbessern die thermische Behaglichkeit während der Anheizphase und der gesamten Nutzungszeit. Die Oberflächentemperaturen der Wände sind vergleichsweise höher, so dass sich eine günstigere operative Temperatur im Raum sehr schnell einstellt. Dieser Vorteil ist insbesondere für sehr massive Gebäude und schlecht besonnte Objekte von großer Bedeutung.

\subsection{Möglichkeiten der Energieeinsparung}

Insbesondere für den Gebäudebestand ist mittels thermisch wirksamer Wandbschichtung eine Möglichkeit gegeben, neben den üblichen Energieeinsparmethoden (dicke Dämmschichten an der Gebäudehülle) den Energiebedarf mit sehr wirtschaftlichen Methoden erheblich zu reduzieren. Die kürzere Anheizphase ist sowohl für eine Wohnnutzung wie auch für eine gewerbliche Nutzung von Bedeutung. Bei einer gewerblichen Nutzung sind typischerweise die „Nichtnutzungszeiten“ deutlich größer als bei einer Wohnnutzung, vergleiche 
[22]. Während des Wochenendes oder in Urlaubszeiten wird hier der Heizbetrieb über längere Zeiträume unterbrochen beziehungsweise reduziert, wie dies aus den Untersuchungen der Raumbeheizungen in Kapitel 6.1 hervorgeht.

Der Vorteil der beschriebenen dünnen Vliesschicht ist, dass ohne großen baulichen Aufwand und mit geringeren finanziellen Mitteln ein beachtlicher Erfolg erzielbar ist. Durch die kleine Dämmschichtdicke (ab 1,2 mm) ist eine Renovierung ohne Veränderungen an Steckdosen, Schaltern, Fußsockelleisten, Tür- und Fensterrahmen möglich.

Neben der Anwendung im Gebäudebestand bietet die hier untersuchte kleine Dämmschicht aus Vlies auch Vorteile für den Neubau, denn auch hierbei sind beim üblichen Nutzerverhalten Verbesserungen für die Behaglichkeit sowie Energieeinsparungen zu erzielen. Zudem können Gebäude mit geringen Nutzungszeiten nach der EnEV §1, welche nicht unter die Energieeinsparverordnung fallen, energetisch verbessert werden.

Raumlufttemperaturabsenkungen, welche durch eine Raumlüftung entstehen, können mittels einer Vliesschicht schneller ausgeglichen werden. Dieser Vorteil ist bei Gebäuden, die keine Lüftungsanlage mit einer Wärmerückgewinnung haben gegeben. Bei diesen werden bei einer Fensterlüftung die Raumluft und die angrenzenden Bauteilschichten abgekühlt. Die Vliesschicht verhindert hier die Auskühlung der massiven Bauteilschichten und ermöglicht eine bessere Wiederanheizung des Raumes, vergleiche Kapitel 7.4.

\subsection{Empfehlung zur Anwendung in der Praxis}

Die messtechnisch durchgeführten Vergleiche erfolgten mit 1 bis $4 \mathrm{~mm}$ dicken Vliesschichten an massiven Wänden.

Die Anwendung von Polystyrol-, Polyethylen-Schäumen oder Ähnlichem ist ebenso möglich, in der praktischen Anwendung kann es hierbei jedoch zu Schwierigkeiten in Form von Feuchteeinwirkungen sowie durch mechanische Beanspruchungen kommen, welche als problematisch einzustufen sind. Zudem wird die Sorptionsfähigkeit der Wände bei derartigen Beschichtungen herabgesetzt. Die Folge können Schimmelpilzbildung sowie unerwünscht hohe Raumluftfeuchte sein.

Vor dem Einsatz der Vliesschicht ist stets eine Gebäude- und Nutzeranalyse anzuraten. Eine massive Bauweise sowie eine intermittierende Beheizung sind Voraussetzung, um größere Energieeinsparungen zu erzielen. Der singuläre Einsatz der Vliesschicht in Gebäudeabschnitten und einzelnen Räumen kann je nach Raumnutzung ebenfalls eine Möglichkeit darstellen. 


\section{Literaturverzeichnis}

1. Krec, Klaus. Zur Wärmespeicherung in Baukonstruktionen. [Hrsg.] R. Oldenbourg Verlag München. Gesundheits-Ingenieur. 1993, 1, S. 11-18.

2. Krüger, Horst. Periodische Aufheizung von Gebäudemassen. Gesundheits-Ingenieur. 2010, 2, S. 69-80.

3. Harald Burgmeister, Bruno Keller. Analyse und Darstellung der Wechselwirkung zwischen Klima und Gebäude: Klimaflächen Teil 1: Grundlagen. [Hrsg.] Ernst \& Sohn Verlag. Bauphysik. 1997, 1, S. 1-6.

4. Hilbig, Gerhard. Wärmespeicherung. [Hrsg.] Grünzweig + Hartmann AG. wksb. 1999, Heft 44, S. 22-26.

5. Keller, Bruno. Klimagerechtes Bauen. Stuttgart : Teubner, 1997. 3-519-05080-3.

6. Hauser, Gerd. Die Wirkung der Wärmespeicherfähigkeit von Bauteilen. [Hrsg.] Ernst \& Sohn. Bauphysik. 22. Jahrgang, 2000, 5, S. 308-312.

7. Künzel, Helmut. Bauphysik und Raumklima - Entwicklung in der Vergangenheit. [Hrsg.] SAINT-GOBAIN ISOVER G+H AG. wksb. Sonderausgabe, 2006, 57, S. 3-8.

8. Gertis, Karl. Zehn Jahre Energieeinsparung im Hochbau - Können wir zufrieden sein? [Hrsg.] Grünzweig + Hartmann und Glasfaser AG. wksb. 1986, 20.

9. Fischer, Gerhard. Energetischer Imperativ. [Hrsg.] G + H. wärme kälte schall. 1974, 2.

10. Hauser, Gerd. Energieeffizienz - der wesentliche Lösungsansatz! [Hrsg.] SAINT-

GOBAIN- ISOVER G + H AG. wksb. 2007, 58.

11. Kopp, Michael. Wärmeschutz ist Umweltschutz. [Hrsg.] Grünzweig + Hartmann AG. wksb. 1988, 24.

12. Ehm, Herbert. Bauen für die Zukunft - Klimaprobleme und ihre Auswirkungen auf das Bauen. [Hrsg.] Grünzweig + Hartmann AG. wksb. 1990, 28.

13. Gellert, Roland. Von wärmewirtschaftlichen Zwängen zum Energiepass: eine Jahrhundertaufgabe? [Hrsg.] Ernst \& Sohn. Bauphysik. 2005, 27.

14. Brunner, Conrad U. Sanierung von Altbauten unter energetischen Gesichtspunkten. [Hrsg.] Ernst \& Sohn Verlag. Bauphysik. 1980, Heft 1.

15. Sommer, Klaus. Fachhochschule Köln. [Online] [Zitat vom: 20. Mai 2011.] http://www.f09.fh-koeln.de/fakultaet/personen/profs/klaus.sommer/00580/.

16. Krüger, Horst. Erhöhung der Wirtschaftlichkeit von Heizanlagen durch instationäre Betriebsweise - Temperaturverlauf, Aufheizleistung und Energieeffizienz bei Vollabschaltung des Heizbetriebes. [Hrsg.] R.Oldenbourg Verlag München. GesundheitsIngenieur. 2005, 5.

17. Sautter, Leopold. Die Lage der Dämmschicht - innen oder außen? [Hrsg.] Grünzweig + Hartmann AG. Wärme Kälte Schall. 1967, 2. 
18. Jenisch, Richard. Lehrbuch der Bauphysik. Stuttgart : B.G. Teubner, 1985. S. 121. 19. Stefan, Wirth. Einfluss der Sonneneinstrahlung auf den Wärmebedarf und die Raumlufttemperaturen beheizter Räume. Dortmund : Deutscher Kälte- und Klimatechnischer Verein , 1994. S. 35.

20. Wolfgang M. Willems, Kai Schild, Simone Dinter, Diana Stricker. Formeln und Tabellen Bauphysik. s.I. : Vieweg \& Sohn Verlag, 2007. S. 37.

21. Hohmann, Setzer, Wehling. Bauphysikalische Formeln und Tabellen. München : Werner Verlag, 2004. S. 94-96.

22. Hauser, Gerd. Einfluß des Wärmedurchgangskoeffizienten und der

Wärmespeicherfähigkeit von Bauteilen auf den Heizenergieverbrauch von Gebäuden.

[Hrsg.] Wilhelm Ernst \& Sohn. Bauphysik. 1984, 5, S. 181.

23. - Einfluß des Wärmedurchgangkoeffizienten und der Wärmespeicherfähigkeit von Bauteilen auf den Heizenergieverbrauch von Gebäuden. [Hrsg.] Ernst \& Sohn.

Bauphysik. 1984, 6.

24. Wikibooks. [Online] [Zitat vom: ] http://de.wikibooks.org.

25. Erwin Knublauch, Erich Cziesielski. Bauphysik. Bautabellen für Ingenieure. s.I. : Werner Verlag, 2004.

26. Keller, Bruno. Klimagerechtes Bauen. Stuttgart : Teubner, 1997.

27. Stefan Plesser, Ernesto Kuchen, M. Norbert Fisch. Ergebnisse einer Evaluierung von Energiekonzepten für Bürogebäude und Schlussfolgerungen für die Energieeffizienz und das Raumklima. [Hrsg.] Ernst \& Sohn Verlag. Bauphysik. 2008, Heft 6.

28. Runa Tabea Hellwig, Wolfgang Bischof. Gültigkeit thermischer Behaglichkeitsmodelle. [Hrsg.] Ernst \& Sohn Verlag. Bauphysik. 2006, Bd. Heft 2. 29. Klaus Sedlbauer, Andreas Holm, Hartwig M. Künzel, Philip Leistner, Klaus Breuer. Raumklima und Innovation - Eine Aufgabe der Bauphysik. [Hrsg.] SAINTGOBAIN ISOVER G+H AG. wksb. 2006, 57.

30. Reinders, H. Mensch und Klima. Düsseldorf : VDI-Verlag GmbH, 1969.

31. Peters, Reinhard, et al., et al. Optimierung der thermischen Behaglichkeit im Bestand. Weinheim : Ernst \& Sohn, 2010. Bd. 5, 32. ISSN 0171-5445.

32. 7726, DIN EN ISO. Umgebungsklima. Instrumente zur Messung physikalischer Größen. Berlin : Beuth Verlag, 2002. Deutsche Fassung EN ISO 7726:2001.

33. 7730, DIN EN ISO. Ergonomie der thermischen Umgebung. Berlin : Beuth, 2006.

34. EN ISO 7730 Anhang C Abschätzung des Isolationswertes von Bekleidungskombinationen. Berlin : s.n., 2005. S. 35.

35. EN ISO 7730 Anhang B, Energieumsätze verschiedener körperlicher Tätigkeiten . 2005.

36. 12831, DIN EN. Heizungsanlagen in Gebäuden, Verfahren zur Berechnung der 
Norm-Heizlast. Berlin : Beuth, 2003.

37. Verordnung über energiesparenden Wärmeschutz und energiesparende Anlagentechnik bei Gebäuden (Energieeinsparverordnung - EnEV). 24.Juli 2007.

38. Verordnung über energiesparenden Wärmeschutz und energiesparende Anlagentechnik bei Gebäuden (Energieeinsparverordnung - EnEV). 18. Juni 2008. 39. DIN 4701 Wärmebedarf von Gebäuden. 1983.

40. Krischer, $O$ und Kast, W. Zur Frage des Wärmebedarfs beim Anheizen selten beheizter Gebäude. Gesundheits-Ingenieur. 1957, Bd. 78.

41. 7726:2001, DIN EN ISO. Umgebungsklima - Instrumente zur Messung physikalische Größen. Anhang A. 2002. S. 17.

42. Sommer, Klaus. http://www.f09.fh-koeln.de. [Online] 2005. [Zitat vom: 21. Juni 2011.] http://www.f09.fh-koeln.de/imperia/md/content/personen/ sommer_klaus/lehrveranstaltung/aufheiz.pdf. 


\section{Lebenslauf}

\section{Alexander Siebel}

Karl-Kuck-Straße 66

52078 Aachen

geboren am 30.11.1978 in Aachen

verheiratet, 1 Kind

Telefon: $\quad 0241 / 95452396$

Telefax: $\quad 0241 / 95452398$

E-Mail: alexandersiebel@googlemail.com

\section{Beruflicher Werdegang}

Seit 10/2010

\section{Lehrauftrag an der Fachhochschule Aachen}

- Demonstration von Labortätigkeit und Messungen in und an Objekten im Bereich Bauphysik

- Betreuung von Diplom- und Bachelorarbeiten

- Feuchtesorptionsversuche an Beschichtungen (Patrick Hövekamp; 2010)

- Ungedämpfte und gedämpfte Schwingungsübertragung bei Masse-Feder-MasseSystemen (Marc Sieberns; 2010)

- Zu den Problemen der messtechnischen Bestimmung der dynamischen Steifigkeit von Estrich-Dämmstoffen (Sabrina Maliska; 2010)

- Untersuchung der Schallabsorption an kleinen Proben (Steffen Paclik; 2011)

- Wärmetechnisches Verhalten von Außenwänden - abhängig von der Dämmstoffdicke und -anordnung (Patrick Thomas; 2011)

- Effizienz der Dämmstoffanordnung bei Außenwänden (Christoph Staaks; 2011)

- Einfluss des Außenklimas auf ausgewählte Fassadensysteme (Carsten Schreinemachers; 2012) 
- Bauakustische Messungen im Labor

- Prüfung der Schallabsorption im Labor

- Erstellung von Immissionsschutzgutachten

- Raumakustische Messungen und Beratungen

- Bauakustische Messungen und Beratungen

- Thermische Messungen im Objekt

- Untersuchung des Raumklimas

\section{Akademische Ausbildung}

09/2000 - 04/2007 Studium Bauingenieurwesen

an der Universität Siegen

mit dem Titel Diplom-Ingenieur

Diplomthema:

Einflüsse von Estrichplattengröße, -gewicht und -verformung auf den gemessenen Trittschallschutz bei schwimmenden Estrichen

\section{Schulische Ausbildung}

von 1984

Grundschule

Sekundarstufe I

Sekundarstufe II 